\title{
RISK AND EMOTION: MEASURING THE EFFECT OF EMOTION AND OTHER VISCERAL FACTORS ON DECISION MAKING UNDER RISK
}

\author{
by \\ Michael Geoffrey Mihalicz \\ Bachelor of Commerce, 2016, Ryerson University \\ Presented to Ryerson University \\ in partial fulfillment of the \\ requirements for the degree of \\ Master of Science in Management \\ in the program of \\ Master of Science in Management
}

Toronto, Ontario, Canada, 2019

(C) Michael Geoffrey Mihalicz, 2019 


\begin{abstract}
AUTHOR'S DECLARATION AUTHOR'S DECLARATION FOR ELECTRONIC SUBMISSION OF A THESIS

I hereby declare that I am the sole author of this thesis. This is a true copy of the thesis, including any required final revisions, as accepted by my examiners.

I authorize Ryerson University to lend this thesis to other institutions or individuals for the purpose of scholarly research.

I further authorize Ryerson University to reproduce this thesis by photocopying or by other means, in total or in part, at the request of other institutions or individuals for the purpose of scholarly research.

I understand that my thesis may be made electronically available to the public.
\end{abstract}




\section{ABSTRACT \\ Risk and Emotion: Measuring the Effect of Emotions and Other Visceral Factors on Decision Making Under Risk}

Michael Geoffrey Mihalicz; Master of Science in Management, Ryerson University (2019)

The science of modelling choice preferences has evolved into an interdisciplinary field contributing to several branches of microeconomics and mathematical psychology. As theories in decision science and related fields mature, descriptive theories have emerged to explain systematic violations of rationality through cognitive mechanisms underlying the thought processes that guide human behaviour. Cognitive limitations are not, however, solely responsible for systematic deviations from rationality and there is a growing body of literature exploring the effect of visceral factors as the more dominant drivers.

This study builds on the existing literature by investigating the impact of anger, sadness, happiness, anxiety, hunger, energy, tiredness and stress on three distinct elements that define risk preference: utility, decision weights and loss aversion. By decomposing the impact of visceral factors on risk preference, I am able to provide evidence supporting the proposition that a portion of the variability in individual choice preferences can be explained by interacting visceral states.

My findings suggest that visceral factors have the strongest effect on loss aversion, which is a major factor in how people code and evaluate financial outcomes. Anger, sadness, happiness, anxiety, energy and tiredness each affect five or more of the model parameters, while hunger and stress are significant only in their interaction with other visceral factors. I also provide evidence to show that the generalized approaches to characterizing visceral factors and risk preference are too broad to be descriptively meaningful. The results of this study show that emotions and other drive states effect the way people process and interpret information, which is crucial in informing decision-makers of the sources and consequences of irrational behaviour.

These findings will be of immediate interest to wealth management specialists, public relations advisers as well as to engineers in designing socially intelligent machines capable of interacting more effectively with humans. 


\section{ACKNOWLEDGEMENTS}

My ultimate gratitude goes to my thesis supervisor, Aziz Guergachi, for his guidance, and for providing me the freedom to pursue this topic. His support throughout my career as a master's student kept me focused and has motivated me to the successful completion of my thesis. His decades of experience and innovative ideas provided me with a unique perspective and challenged me to think differently.

I would also like to thank Steve Gedeon and Sara Thompson, who helped to make my thesis journey possible. Steve Gedeon's attention to detail and faith in my ability to overcome obstacles was instrumental. Sara Thompson helped immensely with her personal, professional and emotional support, and in helping me to reconnect with society and with myself.

I am also deeply indebted to Kevin McInnes, Lurana Kruchten, Heather Mitchell and all the amazing professors at Ryerson who helped me break down barriers to education and achieve what no one thought possible. I would also like to thank Kelly McHale, Sean Wise and everyone in the Entrepreneurship and Strategy department who helped me grow both personally and professionally.

Along my journey, I benefitted greatly from discussions with Julian Appel, Karim Keshavjee, Ijaz Rauf, Hong Yu, Yuanshun Li, Sudeep Bhatia, Sulaimon Giwa, Martin Pyle and David Lewis. Special thanks are also due to Charles Davis and Michel Kouadio at the Faculty of Communication and Design and to everyone at the MLC for their generosity in granting me access to their facilities and equipment, and for creating an inspiring and engaging atmosphere.

Lastly, I am forever grateful to my family who helped me find and stay on the right path. All my accomplishments are a tribute to them. 


\section{DEDICATION}

To my loving family whose patience and faith in me are my tether to hope for a brighter future. 


\section{TABLE OF CONTENTS}

Page

AUTHOR'S DECLARATION ................................................................................. ii

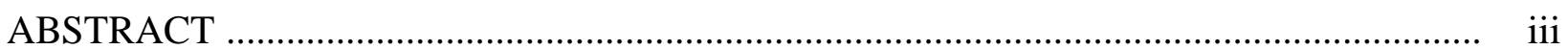

ACKNOWLEDGEMENTS ....................................................................................... iv

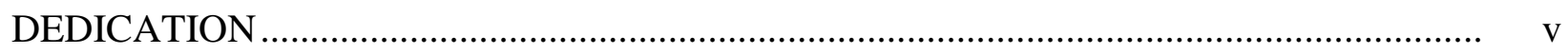

LIST OF TABLES ….................................................................................. ix

LIST OF FIGURES

LIST OF APPENDICES .................................................................................. xii

\section{CHAPTER}

1 INTRODUCTION

1.1 The Current State of the Art ...................................................... 1

1.2 Thesis Objective .................................................................... 3

2 BACKGROUND

2.1 The Origins of Decision Science ................................................... 4

2.2 Ordinal versus Cardinal Utilities .................................................... 5

2.3 The Impact of John von Neumann \& Oscar Morgenstern ............. 7

2.4 Normative versus Descriptive Decision Theory …........................ 8

2.5 The Falsificationism Era ............................................................ 9

3 DETERMINANTS OF RISK PREFERENCE

3.1 The Effect of Stable Factors on Risk Preference ........................... 12

3.1.1 Self-Assessment Measures of Risk Preference ......................... 12

3.1.2 Elicited Risk Preferences ........................................................ 14

3.2 The Effect of Emotions on Risk Preference .................................. 16

3.3 Approaches to Measuring Affective States..................................... 17

3.3.1 Dimensional Approach ....................................................... 18

3.3.2 Distinct-States Approach .................................................... 19

3.3.3 Distinct-States Approach Assuming CPT ................................. 20

3.4 The Effect of Other Visceral Factors on Risk Preference............... 21

3.4.1 Hunger.............................................................................. 21

3.4.2 Sleep Deprivation .............................................................. 22

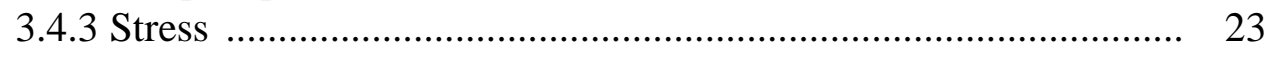

3.5 Visceral Factor Interactions ................................................... 24

3.6 Self-Assessed Measures of Visceral Factors .................................. 25 
4.1 Minimum Considerations ............................................................ 26

4.1.1 Nonlinear Treatment of Outcomes and Probabilities.................. 26

4.1.2 Reference Points ................................................................ 27

4.1.3 Monotonicity ................................................................... 28

4.2 Cumulative Prospect Theory ...................................................... 28

4.3 Parametric Specification ..................................................... 31

4.3.1 Power Parameter ................................................................... 32

4.3.2 Decision Weight Parameter .................................................... 33

4.3.3 Loss Aversion Parameter ......................................................... 36

4.3.4 Empirical Findings ............................................................ 38

5 METHODOLOGY

5.1 Survey Design .................................................................................. 40

5.2 Data Collection.......................................................................... 40

5.3 Structure of Risk Preference Elicitation Questions......................... 41

5.4 Basic Utility Elicitation Method .................................................. 42

5.5 Decision Weight Elicitation Method .............................................. 44

5.6 Loss Aversion Elicitation Method .................................................. 45

5.7 Measuring Visceral Factors .................................................. 48

5.8 Measuring Personality Traits ........................................................ 49

6 ANALYSIS AND RESULTS

6.1 Demographic Variable Summary Statistics ................................ 51

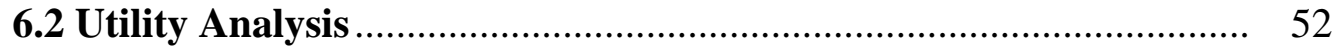

6.2.1 Non-Parametric Utility Analysis............................................ 52

6.2.2 Parametric Utility Analysis .................................................... 55

6.3 Decision Weight Analysis ........................................................ 56

6.3.1 Non-Parametric Decision Weight Analysis .............................. 56

6.3.2 Parametric Decision Weight Analysis ..................................... 58

6.4 Loss Aversion Analysis............................................................ 60

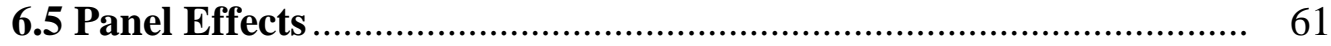

6.5.1 Reliability Analysis ........................................................... 61

6.5.2 Fixed Effects Regression ................................................. 63

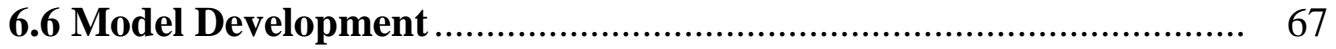

6.6.1 Dimension Reduction........................................................... 67

6.6.2 Variable Selection ............................................................... 69

6.6.3 Case Removal .................................................................... 70

6.6.4 OLS Regression Models ..................................................... 71

6.6.5 Visceral Factor Effects ......................................................... 74

6.7 Hierarchical Regressions ....................................................... 77

6.8 Reliability of Stable Determinants as Classifiers ............................ 78

7 DISCUSSION, APPLICATIONS AND CONCLUSION

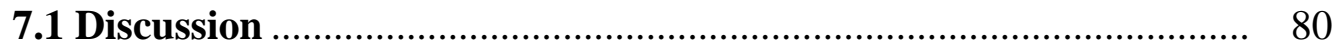

7.1.1 The Significance of Interaction Effects ................................. 80

7.1.2 Overgeneralization of Visceral Factors and Risk Preference ...... 81 
7.2 Applications.............................................................................. 82

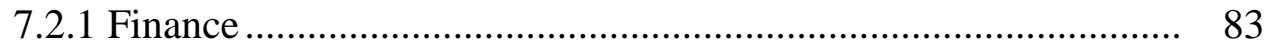

7.2.2 Machine-Human Interaction .................................................... 85

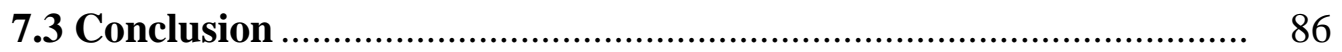

8 LIMITATIONS AND FUTURE WORK

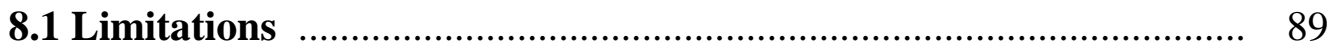

8.1.1 Data Collection and Cleaning .................................................. 89

8.1.2 Elicitation Methods ................................................................ 89

8.1.3 Compensation........................................................................ 90

8.2 Future Work .............................................................................. 91

\section{APPENDICES}

A Expected Utility Theory: Axiomatic Foundation ……….................................. 93

B Stable Determinants of Risk Preference .............................................................. 93

C Illustration of the Survey Presented to Participants.............................................. 94

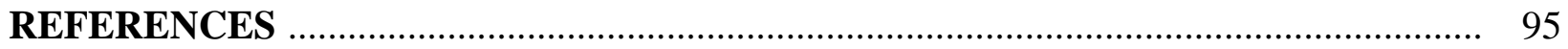




\section{LIST OF TABLES}

Table

Page

1 Significance of Demographic Factors on Self-Assessed Risk Preference .................... 13

2 Significance of Demographic Factors on Elicited Risk Preference ............................ 14

3 Median Empirical Estimates of the Model Parameters ............................................. 38

4 Average Time to Complete Each Trial of the Experiment ........................................ 41

$5 \quad$ Structure of Risk Elicitation Questions .................................................................... 42

6 Visceral Factor Summary Statistics ...................................................................... 49

$7 \quad$ Personality Trait Summary Statistics ……………................................................ 50

$8 \quad$ Numeric Demographic Variable Summary Statistics .............................................. 51

9 Categorical Demographic Variable Summary Statistics ........................................... 51

10 Pooled Sampled T-Test of Successive Differences ................................................... 53

11 Non-Parametric Utility Curvature Frequencies ........................................................ 54

12 Individual-Level Non-Parametric Utility Curvature ………..................................... 54

13 Sample Statistics for the Power Parameter in the Gain and Loss Domains ................. 55

14 Parametric Utility Curvature Frequencies............................................................... 55

15 Individual-Level Parametric Utility Curvature …….................................................. 56

16 Summary Statistics for the Elicited Probabilities...................................................... 57

17 Significant Differences from Linearity …………............................................. 58

18 Summary Statistics for the Decision Weight Parameters ………................................ 59

19 Summary Statistics for the Loss Aversion Index ..................................................... 61

20 Variables of Interest and Tests of Within-Subject Changes ....................................... 62 
21 Tests of Within-Subjects Changes Over the Three Trials..................................... 62

22 Related-Samples Friedman's Two-Way ANOVA, and All Pairwise Comparisons .... 63

23 Time- and Entity-Fixed Effects Regressions .................................................... 65

24 Rotated Component Loadings from the NLPCA .............................................. 69

25 Summary of Exploratory Analyses ........................................................... 70

26 Sample Sizes and Excluded Cases by Section ................................................ 71

27 OLS Regression Models for each of the Seven Parameters................................... 71

28 Hierarchical (Blockwise Entry) Regression Summary ....................................... 78

29 Pooled OLS vs. Random-Effects ................................................................. 79 


\section{LIST OF FIGURES}

Figure

Page

1 Two Choice Prospects Illustrating the Common Consequence Effect ...................... 9

2 Number and Proportion of Articles Referencing Emotion, Affect and/or Mood......... 16

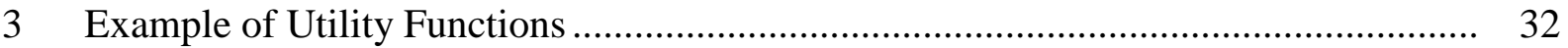

4 Changes in the Elevation Parameter on the Probability Weighting Function............. 34

5 Changes in the Curvature Parameter on the Probability Weighting Function ............. 35

$6 \quad$ Empirical Estimates of the Probability Weighting Function .................................. 36

$7 \quad$ Example of a Risk Elicitation Question .............................................................. 40

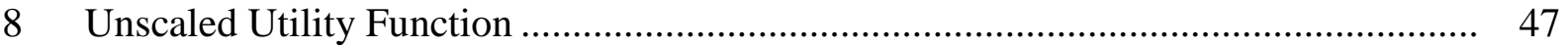

9 Visceral Factor Self-Assessment Method ......................................................... 48

10 Plot of the Area Under the Utility Curve ............................................................. 54

11 Median Decision Weights with Linear Overlay................................................... 57

12 Median Probability Weighting Functions ........................................................ 60

13 Average Magnitude of Significant Visceral Factor Effects .................................... 72

14 Illustration of the Survey Presented to Participants .................................... 94 


\section{LIST OF APPENDICES}

Appendix $\quad$ Page

A Expected Utility Theory: Axiomatic Foundation .............................................. 93

B Stable Determinants of Risk Preference ......................................................... 93

C Illustration of the Survey Presented to Participants ............................................. 94 


\section{CHAPTER 1 \\ INTRODUCTION}

\subsection{The Current State of the Art}

Many theories have been proposed that seek to model the cognitive mechanisms underlying decision-making, but few attempt to specify the role of our more primal drive states. Visceral factors, such as hunger, thirst, sexual desire, physical pain and emotions, are essential to human evolution and fitness, and understanding how and why they alter perceptions can provide valuable insights into human behaviour. Depending on the intensity of the desire, visceral factors can magnify the anticipated rewards of a choice or action and are thought to have the power to override rational deliberation entirely (Loewenstein, 1996). By incorporating the effect of visceral factors in a popular cognitive theory of decision making under risk and uncertainty, this study seeks to gain insight into the way people process and interpret information and guide more effective decision-making by informing decision-makers of several sources and consequences of irrational behaviour.

The scientific study of human decision making began with Expected Utility Theory (EU), the roots of which can be traced back to a group of mathematicians almost three centuries ago who sought to establish a more practical framework for guiding decision making (Bernoulli, [1738] 1954). Over two hundred years later, the concept of utility gained widespread notoriety with the release of a book titled 'Theory of Games and Economic Behaviour' (von Neumann \& Morgenstern, 1944). In it, von Neumann \& Morgenstern effectively mathematized the elusive notion of utility through a set of normatively appealing axioms that enabled economists to treat utilities as numerically measurable quantities and maximize representations of preference relations. The axioms they developed were so widely accepted that EU became a staple in economic theory and a standard for individual choice. This, however, was never von Neumann \& Morgenstern's intention; for them, EU was merely a starting point which they expected would eventually be replaced as the base of empirical evidence developed.

Many of the early discussions surrounding the axiomatic approaches to constructing decision theories revolved around the notion of rationality and assumed that people were rational (Bernoulli, [1738] 1954; Friedman \& Savage, 1948; Tversky \& Kahneman, 1979). Early 
pioneers in the field such as von Neumann \& Morgenstern (1944) postulated that human decision making followed guiding principles founded on the logic of rationality and any decision violating these principles was inherently unpredictable. This notion was challenged as systematic and often intentional violations of the principles of rationality were increasingly observed in empirical tests (Allais, 1952; 1979; Simon, 1956). These were initially explained as cognitive limitations that occur naturally when the mind is confronted with unmanageably large amounts of information. Among those who contested the presumption of rationality was Herbert Simon who proposed that all human decision-making is at most boundedly rational and inherently subject to limitations. The idea that people are boundedly rational implies that people strive to make rational decisions but are limited in their ability to do so by factors such as emotions and cognitive limitations (Simon, 1956).

Many recent theories including prospect theory (Kahneman \& Tversky, 1979) seek to model cognitive mechanisms thought to be responsible for observed regularities in decision making and use empirical tests to validate the existence of the association. These theories are much better able to describe how people make decisions and have been instrumental in developing our understanding of the nature of decision making and the mechanisms underlying the deliberation process. With advancements in the scientific study of the mind and intelligence, descriptive decision theories have focused on accounting for cognitive mechanisms with little regard for the multitude of other factors systematically affecting decisions.

Prospect theory was the first widely accepted cognitive theory to abandon the conventional axiom-based approach to modelling decision-making in favour of a procedural approach. This theory applies a series of edits that simplify decision tasks prior to evaluating prospects and is better able to explain observed regularities in decision making under risk (Camerer, 1989; Harless \& Camerer, 1994). In response to appeals from the academic community, Tversky and Kahneman (1992) developed a new version of prospect theory called cumulative prospect theory (CPT) which can be applied to decisions involving any number of outcomes with known or unknown probabilities. CPT meets the minimum criteria for a descriptive theory of choice to be considered adequate (Tversky \& Kahneman, 1992) and has been used to explain several economic anomalies (Thaler, 1980; Kahneman, Knetsch \& Thaler, 1991). CPT was selected for the present study because of its ability to capture the cognitive limitations in decision making 
under risk, it satisfies the criteria considered to be minimal requirements for a descriptive theory of choice and because it is completely observable using non-parametric methods.

While it is understood that cognitive limitations have a significant influence on decision making, they are not solely responsible for systematic deviations from rationality and many are now claiming that emotions and other visceral factors are the more dominant drivers (Loewenstein et al. 2001; Lerner \& Keltner, 2001).

\subsection{Thesis Objective}

The present study seeks to gain a deeper understanding of the limits of human rationality by measuring the effect of different types and levels of arousal on individual risk preference. Two questions are of primary interest in this study:

1. Which visceral factors and combinations of visceral factors are significant determinants of the parameters that characterize risk preference under cumulative prospect theory?

2. Why and through what mechanisms do visceral factors affect decision-making under risk?

The goal of these experiments is to introduce a new method for collecting data and to identify significant limiting factors to rationality often unaccounted for in conventional decision theories. By decomposing risk preference into unique, separable model parameters I'm able to provide a series of measurable and systematic effects on the subjective interpretations of gamble attributes and evidence supporting the proposition that a portion of the variability in human preferences unaccounted for by cognitive limitations can be explained by interacting visceral states. In doing so, I offer practitioners a means to improve the predictive utility and practical applicability of CPT and insight into some possible sources of irrational decision making. 


\section{CHAPTER 2 \\ BACKGROUND}

\subsection{The Origins of Decision Science}

The history of decision science began in the 1700s when a group of mathematicians sought out to rationalize a paradox that became known as the St. Petersburg paradox (Bernoulli, [1738] 1954). At the time, the fair price for any gamble was considered its expected value (EV). This was calculated by summing all the possible outcomes, each of which was multiplied by the number of states in which that outcome would be obtained and divided by the total number of relevant states:

$$
\frac{a X_{1}+b X_{2}+\cdots+k X_{l}}{\sum_{i=1}^{n} S_{i}}
$$

where $\left[a=\sum_{i=1}^{n} S_{i} \mid X_{1}\right],\left[b=\sum_{i=1}^{n} S_{i} \mid X_{2}\right]$ or rather that $a$ is the sum of the number of states in which $X_{1}$ occurs, $b$ is the sum of states in which $X_{2}$ occurs, and so on. $\sum_{i=1}^{n} S_{i}$ is the total number of states that result in at least one outcome $\left[X_{1}, X_{2}, \ldots, X_{l}\right]$. If we consider a coin flip for $\$ 100$ or nothing, where $X_{1}=\$ 100, X_{2}=\$ 0, a=\sum_{i=1}^{n} S_{i}\left|X_{1}=1, b=\sum_{i=1}^{n} S_{i}\right| X_{2}=1$ and $\sum_{i=1}^{n} S_{i}=$ $a+b=2$, then the equation becomes $1 / 2 \cdot(\$ 100)$.

This equation intentionally assumed that all states were equi-probable and proposed a linear treatment of both probabilities and outcomes. The prevailing sentiment in the early 1700 s was to establish a set of objective and normatively acceptable rules that everyone could follow to determine the value of a gamble and that persons of authority could use to deliberate. Daniel Bernoulli ([1738] 1954) rejected the idea that people should strictly adhere to this model and developed an alternative theory under the pretence that wealth affects the sensibility of accepting risk. In doing so he introduced the concept of the utility of wealth. The utility of wealth was presented as a non-linear transformation of gamble outcomes where each additional dollar provided marginally less utility. Bernoulli ([1738] 1954) proposed that the principle of the marginal utility of wealth be integrated into the existing EV framework by replacing the outcomes with their respective utility values and maintaining the assumption of equi-probability of states: 


$$
\frac{a b \log \frac{W_{1}}{W_{0}}+c b \log \frac{W_{2}}{W_{0}}+\cdots+k b \log \frac{W_{l}}{W_{0}}}{\sum_{i=1}^{n} S_{i}}=\frac{a U\left(W_{1}\right)+c U\left(W_{2}\right)+\cdots+k U\left(W_{l}\right)}{\sum_{i=1}^{n} S_{i}}
$$

where $W_{i}$ represents a potential future wealth state and $b$ is a constant indicating the degree of curvature of the utility function given an initial wealth state, $W_{0}$.

Though Bernoulli acknowledged that the utility of outcomes may vary depending on the individual or circumstances, he considered such situations to be rare exceptions and proposed that utility of outcomes be universally defined as continuous, non-decreasing in wealth and a product of the amount of goods one already possesses in combination with the present worth of their earning power, $W_{0}$. There are two notable implications of this theory. First, it implies global risk aversion meaning people are averse to risk at every potential wealth state. Second, the disutility of losing an actuarially fair gamble will always exceed the utility of winning, implying that all actuarially fair gambles are irrational. This did not, however, preclude wagering because the amount of compensation that the theory predicts people will demand to assume risk varies with wealth. Bernoulli used this to demonstrate how individuals can reach insurance agreements as well as how the theory could be used to optimize a diversification strategy.

Bernoulli's paper was the first published account of EU and would eventually form the foundation of modern decision science. Since then, EU has had a tremendous impact on how people evaluate risk and has been instrumental in the advancement of several fields including game theory (von Neumann \& Morgenstern, 1944), portfolio theory (Markowitz, 1991), mathematical psychology (Batchelder, 2010) and behavioural economics (Camerer, 1999).

\subsection{Ordinal versus Cardinal Utilities}

While Bernoulli's theory was often referenced and highly credited, the concept of individual utilities was not integrated into decision theories in any meaningful way until the mid-twentieth century. From the late nineteenth century until the mid-twentieth century there was an extensive debate concerning the measurability of utility that resulted in a clear divide between two distinct schools of thought: ordinalists and cardinalists.

Most theories in decision science propose a nonlinear treatment of outcomes and, since there is no universal understanding of the nature of utility, a central issue in the evolution of 
argumentation revolves around the epistemology of utility. The definition of cardinal utilities in use today was first proposed by Samuelson (1938) who defined utilities as unique up to positive linear transformations: $a U(x)+b$, where $a>0$. This definition allows the ranking of both prospects and differences between prospects making possible the determination of relative preference strengths. Ordinal utilities, on the other hand, define utilities as unique up to orderpreserving transformations: $f[U(x)]$, where $f^{\prime}>0$, which only allows for the ranking of prospects. Since ordinal utilities allow for nonlinear transformations of the representing function, prospects could no longer be considered any degree better or worse than other prospects and suggests that the analysis of choice preferences rely solely on a prospect's rank amongst alternatives. Ordinalists believe there to be a whole class of equivalent utility functions that can represent demand behaviour similarly and do not acknowledge a need to be more specific. While there are many who believe that cardinal utilities emerged in the late nineteenth century, not long before the ordinalist revolution, the history of this debate dates back much further and is relatively abstruse.

The ordinalist revolution was initiated under the pretence that it was not possible to measure utility objectively and that economic theory does not require it to be. Ordinalists in the early twentieth century adopted a logical positivist approach, rejecting cardinal utility on the basis that utilities cannot be objectively measured. There is, however, a contradiction in that many of the economic analyses using ordinal utilities rely on principles that imply a form of cardinality. These include the concept of diminishing marginal utility and the differentiability of indifference curves. Cardinality was therefore not completely abandoned with the introduction of the ordinal approach and, in fact, Pareto himself allowed consumption transitions to be ranked in special cases (Pareto, [1909] 1971).

The economic community seemed to embrace the ordinalist perspective (Pareto, [1898] 1966), but there has always been an undercurrent of cardinalists not willing to abandon the ranking of preference transitions and the information therein (Moscati, 2013a; 2013b). John von Neumann and Oskar Morgenstern popularized cardinal utility representations in 1944 by presenting an axiomatic foundation for EU and introducing a new framework to solve games that involve a degree of strategic interdependence. 


\subsection{The Impact of John von Neumann \& Oscar Morgenstern}

Both Bernoulli ([1738] 1954) and von Neumann \& Morgenstern (1944) adopted a critical rationalist approach in presenting arguments for the existence of a utility function that transforms the gamble outcomes according to an individual's wealth. One of the reasons von Neumann and Morgenstern's book made such an impact is because it proposed that people acted under a maxim which implied that human decision making could be predicted. von Neumann \& Morgenstern used a deductive approach to modelling decision making by outlining a set of rules or axioms based on the principles of rationality that people were believed to follow or should follow when making decisions. These axioms formed a basis for conventional theories and enabled researchers to test and compare theories. Theories that were presented without axiomatic foundations were often treated as untestable or noncredible theories and were generally less accepted in the academic community. This was believed to be the case with Becker \& Sarin's (1987) lottery dependent utility theory which was absent from the popular empirical investigations that compared many of the theories from this era (Schmidt, 2001; Hey \& Orme, 1994; Harless \& Camerer, 1994). For a description of the axiomatic foundation of EU and their implications, readers are referred to the section 'Expected Utility Theory: Axiomatic Foundation' in Appendix A.

In their book, von Neumann \& Morgenstern adopted an objective approach to characterizing probabilities that assumed the linear treatment of explicitly stated probabilities. This was to better facilitate the development of a numerical estimation of utility and intentionally left probability transformations open for further research. The following is a mathematical representation of EU:

$$
E(U)=\sum_{i=1}^{n} p_{i} \cdot u\left(x_{i}\right)
$$

where $p_{i}$ is the explicitly stated probability that the state resulting in outcome $x_{i}$ will occur for prospect $i$ and $U\left(x_{i}\right)$ is a function reflecting the nonlinear treatment of outcome $x_{i}$.

Though consistent with the period's standard definition of utility, extensive studies have established a general tendency towards loss aversion which raises concerns about the fundamental assumption that utility is solely a function of cumulative wealth (Markowitz, 1952; Kahneman \& Tversky, 1979; Camerer, 2005). EU also assumes universality of preferences 
dependent on wealth which is hard to defend in the face of consistent choice preferences amongst wealth classes. Lastly, von Neumann \& Morgenstern (1944) also claim that all choices involving risk can be valued in monetary terms and that preferences between these amounts are synonymous with individual preferences involving pure risks. This assumption is essential to their attempt to generalize the hypothesis across the economic domains that are the focus of their theory. While their book does not provide evidence to support this generalization, it does account for it by including a qualifier limiting predictions to pure risk factors.

\subsection{Normative versus Descriptive Decision Theory}

Following the characterization of choice preferences as mathematical functions by von Neumann and Morgenstern (1944), the field of decision theory divided again into those who sought to develop normative decision theories and those interested in describing how people actually make decisions. Descriptive decision theory emerged as empirical testing of EU and its axioms exposed weaknesses in the theory.

One of the initial concerns involved the existence of both gambling and insurance meaning that people could be risk seeking under some circumstances and risk averse under others. The presence of both gambling and insurance cannot be explained by a univariate model implying global risk preference and drove many scholars to seek alternative explanations. Friedman \& Savage (1948) believed that the co-existence of insurance and gambling could provide the answers necessary to develop a more accurate theory for choice preferences, a glimpse into the nature of human behaviour as well as a benchmark for testing hypotheses concerning actual economic behaviour. Friedman \& Savage adopted a positivist approach to their work, believing that general laws persisting across cultures and time govern attitudes towards risk in decision making. This was presented as an inductive argument drawing from observed regularities in consumer behaviour and economic domains to better understand risk preferences and to construct a hypothesis pertaining to the shape and nature of individual utilities.

Friedman \& Savage (1948) maintained many of the fundamental principles presented in EU including the cardinality, generalizability and quantifiability of choice preferences, the assumption of expected utility maximization, and the representation of utility as an increasing function of income - but propose a different shape to the utility function that is more consistent with observed regularities. Though many of the assumptions presented in this paper were 
ultimately disproven through empirical testing, the assumptions and elicitation methods described provided not only a basis for confirming or rejecting theories, but also a method for measuring utility and validating descriptive decision theories. In making fundamental aspects of decision theories testable, this paper helped shift the nature of argumentation from a deductive to inductive reasoning and has proven fundamental to the advancement of decision science.

\subsection{The Falsificationism Era}

Though there had been attempts to develop methods of measuring utility prior to the revival of EU by von Neuman and Morgenstern in 1944 (Thurstone 1927; Horst 1932), some of the most significant contributions came shortly after as scholars raced to develop reliable methods to test the EU and its related axioms empirically. The development of elicitation methods acted to shift the methodology of decision theorists to a research-based approach and ushered in an era of falsificationism wherein researchers could test the axiomatic foundations of decision theories. This shifted the field away from logic-based reasoning towards more empirically grounded research.

Among the most famous violations of EU formalized by Maurice Allais $(1953 ; 1979)$ are the common consequence and the common ratio effects. These effects show that preference strengths for prospects are systematically influenced by shifts in the probability mass of a common consequence and a clear violation of the independence axiom proposed in EU (Wu \& Gonzalez, 1998; Starmer \& Sugden, 1989). The common consequence effect is best illustrated using a variation of Allais' example drawn from Wakker (2010) shown in Figure 1.

Figure 1 - Two Choice Prospects Illustrating the Common Consequence Effect
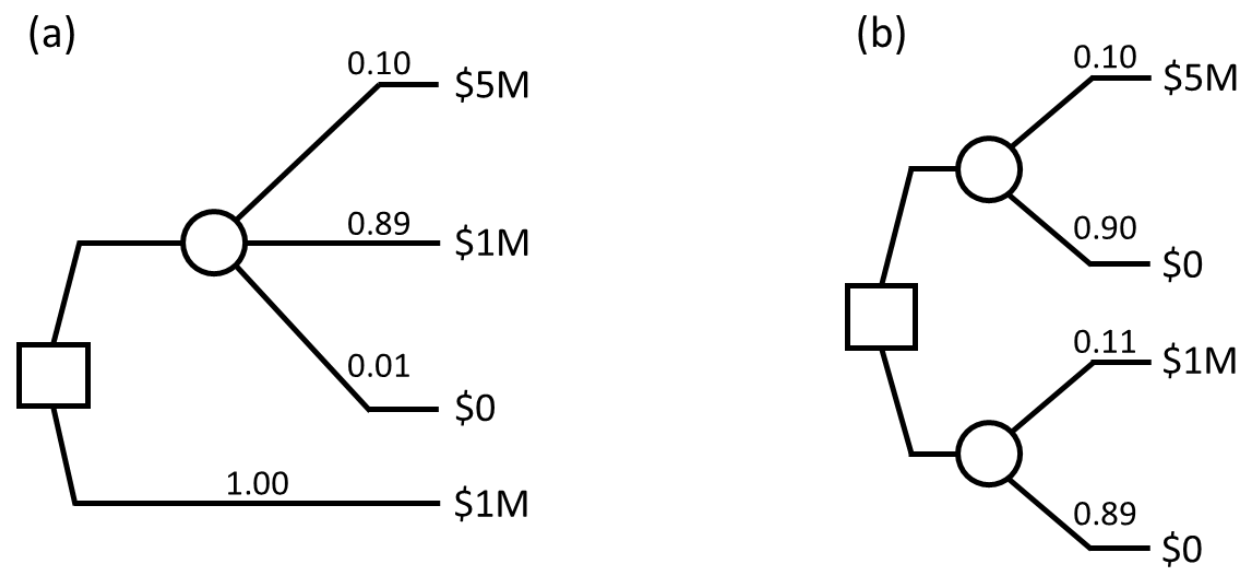
Question (a) asks participants to choose between two prospects: one that will pay \$1 million with certainty and another that will pay $\$ 5$ million with a probability of $0.10, \$ 1$ million with a probability of 0.89 or $\$ 0$ with a probability of 0.01 . Question (b) again asks participants to choose between two prospects only this time there are no certain outcomes in either prospect. The first prospect will pay $\$ 5$ million with a probability of 0.10 or $\$ 0$ with a probability of 0.90 and the second prospect will pay $\$ 1$ million with a probability of 0.11 or $\$ 0$ with a probability of 0.89. Studies have shown that people tend to prefer the prospect with the certain outcome in the first question and the prospect with the higher outcome in the second question (Allais, 1953; 1979; Kahneman \& Tversky, 1979). Assuming EU, this preference relation implies:

$$
\begin{gathered}
u(\$ 1 M)>.1 \cdot u(\$ 5 M)+.89 \cdot u(\$ 1 M)+.01 \cdot u(\$ 0) \\
\& \\
.1 \cdot u(\$ 5 M)+.9 \cdot u(\$ 0)>.11 \cdot u(\$ 1 M)+.89 \cdot u(\$ 0)
\end{gathered}
$$

which reduces to: $.11 \cdot u(\$ 1 M)>.1 \cdot u(\$ 5 M) \& .1 \cdot u(\$ 5 M)>.11 \cdot u(\$ 1 M)$. Paradoxes of this nature are believed to be the result of a special preference people have for certain outcomes, otherwise known as the certainty effect (Kahneman \& Tversky, 1979).

The era of falsificationism was largely initiated by von Neuman and Morgenstern (1947) themselves along with Friedman \& Savage (1948), Mosteller \& Nogee (1951), Markowitz (1952), Alchian (1953) and others who developed a variety of elicitation methods now known as the standard gamble (SG) methods. The basic structures of SG methods take the form,

$$
\left[X_{1}, \alpha, X_{0}\right] \prec, \sim,>Z_{i} \Leftrightarrow \alpha X_{1}+(1-\alpha) X_{0}<,=,>Z_{i}
$$

where, $\alpha$ is a probability measure satisfying $\alpha \in(0,1) ; X_{1}, X_{0} \in X$ represent payouts in a gamble; and $Z_{i}$ represents a certain amount that lies between the two extremes in the gamble, $X_{1}>Z_{i}>X_{0}$. The notation $\left[X_{1}, \alpha, X_{0}\right]$ represents a gamble in which there is a probability of $\alpha$ that the individual will receive $X_{1}$ and a probability of $(1-\alpha)$ that they will receive $X_{0} . \prec, \sim,>$ denote preference relations as follows: if $\left[X_{1}, \alpha, X_{0}\right] \prec Z_{i}$, the decision maker strictly prefers the certain outcome over the gamble; if $\left[X_{1}, \alpha, X_{0}\right] \sim Z_{i}$, the decision maker is indifferent between the gamble and the certain outcome; and if $\left[X_{1}, \alpha, X_{0}\right]>Z_{i}$, the decision maker strictly prefers the gamble over the certain outcome. 
There are four categories of SG methods following this general form: preference comparison, value equivalence (VE), probability equivalence (PE) and certainty equivalence (CE). These methods are used to either check the consistency of a utility function, to establish boundaries for acceptable utility functions or to elicit utility functions directly by making iterative adjustments until the decision maker is satisfied that they have reached their indifference point. VE, PE and CE methods serve the same purpose as the preference comparison method only instead of asking the decision maker to select a preference, these methods establish the value or range of values for a gamble outcome $\left(X_{1}\right.$ or $\left.X_{0}\right)$, probability $(\alpha)$ or certainty equivalent $\left(Z_{i}\right)$, respectively, that satisfy the indifference relation (von Neumann \& Morgenstern, 1947; Friedman \& Savage, 1948; Mosteller \& Nogee, 1951; Markowitz, 1952; Alchian, 1953). Once the outcomes are normalized such that $X_{1}=1$ and $X_{0}=0$, the utility of the certainty equivalent equals the probability of obtaining $X_{1},\left(Z_{i}=\alpha\right)$. The process is then repeated using a chaining or fractile method until a sufficiently complete utility function has been constructed (von Neumann \& Morgenstern, 1944; Torgerson, 1959; Keeney \& Raiffa, 1976). The fundamental assumption in normalizing the outcomes and eliciting utility functions using this method is that utility is unique up to positive linear transformations which implies the cardinality of utility. Readers are referred to Farquhar (1984) for a more detailed account of the SG methods used to elicit single-attribute expected utility functions.

Researchers have also found that certain hybrid methods combining two or more of the SG methods are fairly robust against many types of biases and avoid altogether biases that result from a heuristic rule associated with a particular elicitation method (Farquhar, 1984; Krzysztofowicz \& Duckstein, 1980). By employing different techniques or variations of methods, researchers have been able to mitigate many of the biases inherent in the SG methods (Wakker, 2004a). One pervasive bias known as the certainty effect (Schmidt, 1998), however, requires that researchers abandon the certain outcome, $Z_{i}$, and instead use paired-gamble methods where participants identify an indifference point between two gambles. 


\section{CHAPTER 3 \\ DETERMINANTS OF RISK PREFERENCE}

\subsection{The Effect of Stable Factors on Risk Preference}

Many studies have investigated what drives the variability in individual-level risk preference although not all studies are directly comparable. There are two broad categories of measures of risk preference: one that measures what people believe their risk preference to be and another that elicits risk preference from lengthier questionnaires. While self-assessed risk preferences are broadly consistent with more comprehensive risk preference elicitation methods, they are subject to increased variability and tend to understate risk preference (Hallahan et al. 2004). It is therefore necessary to differentiate between different measures of risk preference when assessing the results of studies exploring the determinants of risk preference.

\subsubsection{Self-Assessment Measures of Risk Preference}

Self-assessed measures of risk preference are readily available through the Survey of Consumer Finances (SCF) conducted by the Federal Reserve Bank every three years in the United States. Participants in this survey identify asset and liability holdings as well as the amount of risk they are willing to accept when making investment decisions. This survey has been used to identify several demographic factors as significant determinants of risk preference including wealth, income, gender, education, race, occupation, years until retirement, marital status, number of dependents and household size (Hawley \& Fujii, 1993; Sung \& Hanna, 1996; Perraudin \& Sorense, 2000; Grable \& Lytton, 1998; Chang, DeVaney \& Chiremba, 2004). The significance of demographic factors explored in these studies are presented in Table 1. 
Table 1 - Significance of Demographic Factors on Self-Assessed Risk Preference

\begin{tabular}{|c|c|c|c|c|c|c|c|c|c|c|c|}
\hline Study & Age & Age $^{2}$ & $\begin{array}{l}\text { Gross } \\
\text { WIth }\end{array}$ & Inc. & Edu. & Gndr & $\begin{array}{l}\text { Marital } \\
\text { Status }\end{array}$ & Race & $\begin{array}{l}\text { Self- } \\
\text { Empl. }\end{array}$ & Other & Source \\
\hline $\begin{array}{l}\text { Hawley \& Fujii, } \\
\text { (1993) }\end{array}$ & $x$ & $x$ & & $\mathrm{X}^{*}$ & $\mathrm{X}^{*}$ & $\mathrm{X}^{*}$ & $\mathrm{X}^{*}$ & $\mathrm{X}^{*}$ & & $\begin{array}{l}\text { Age }(55+)^{*} ; \text { Health } \\
\text { status; net wealth }\end{array}$ & $\begin{array}{l}1983 \\
\text { SCF }\end{array}$ \\
\hline $\begin{array}{l}\text { Perraudin \& } \\
\text { Sorense, (2000) }\end{array}$ & $\mathrm{X}^{*}$ & & $\mathrm{X}^{*}$ & $\mathrm{X}^{*}$ & $\mathrm{X}^{*}$ & & $x$ & $\mathrm{X}$ & & $\begin{array}{l}\text { Occupation; } \\
\text { household size*; } \\
\text { head of household*; } \\
\text { liquidity preference* }\end{array}$ & $\begin{array}{l}1983 \\
\text { SCF }\end{array}$ \\
\hline $\begin{array}{l}\text { Sung \& Hanna, } \\
\text { (1996) }\end{array}$ & $x$ & & $\mathrm{X}^{* * *}$ & $X^{* * *}$ & $X^{* * *}$ & $X^{* * *}$ & $X^{* * *}$ & $X^{* * *}$ & $\mathrm{X}^{* * *}$ & $\begin{array}{l}\text { Yrs until retirement; } \\
\text { household size**; } \\
\text { occupation***; } \\
\text { homeownership*** }\end{array}$ & $\begin{array}{l}1992 \\
\text { SCF }\end{array}$ \\
\hline $\begin{array}{l}\text { Grable \& Lytton, } \\
\text { (1998) }\end{array}$ & $\mathrm{X}$ & & & $X^{* * *}$ & $X^{* * *}$ & $\mathrm{X}^{* * *}$ & $\mathrm{X}^{* * *}$ & $X^{* * *}$ & $\mathrm{X}^{* * *}$ & $\begin{array}{l}\text { Single***; never } \\
\text { married; white*; } \\
\text { black*; hispanic*; } \\
\text { other race; profnl. } \\
\text { status*** }\end{array}$ & $\begin{array}{l}1992 \\
\text { SCF }\end{array}$ \\
\hline $\begin{array}{l}\text { Chang et al. } \\
\text { (2004) }\end{array}$ & $x$ & & & $\mathrm{X}^{* * *}$ & $\mathrm{X}^{* * *}$ & & $X^{* * *}$ & $\mathrm{X}^{* * *}$ & & $\begin{array}{l}\text { Net wealth***; empl. } \\
\text { status*** }\end{array}$ & $\begin{array}{l}2001 \\
\text { SCF }\end{array}$ \\
\hline
\end{tabular}

*** Significant at the .1 percent level

** Significant at the 1 percent level

* Significant at the 5 percent level

One notable factor explored in each of these studies is age. The relationship between age and risk preference is of great interest to many in the academic community as well as to industry professionals. Risk preference was traditionally believed to decrease with age based on the reasoning that decreases in future earning potential should make people more risk averse. While age is not found to be a significant determinant of self-reported measures of risk preference in the reviewed literature, several studies have found age to affect portfolio compositions (Perraudin \& Sorense, 2000; Hanna \& Chen, 1997).

Chang, DeVaney \& Chiremba (2004) investigated the relationship between age and risk preference using data containing the self-reported risk preferences of individuals along with financial information from which the ratio of risky financial assets to net worth could be obtained. This study found the effect of age to be insignificant on self-reported measures of risk preference, though it was a significant determinant of objective risk preference. Additionally, age squared had a significant negative relationship with objective risk preference indicating that the ratio of risky assets to net worth follows a hump-shaped pattern; first increasing and then decreasing with age. 


\subsubsection{Elicited Risk Preferences}

The findings presented by Chang, DeVaney \& Chiremba (2004) are significant because they imply that while people's belief about their own risk preferences do not change, their portfolio compositions do. Knowing that financial decision making changes with age, it follows that selfreports of risk preferences may not be an appropriate tool to better understand the behavioural implications of stable factors on risk preference. Several studies exploring the effect of stable determinants on more comprehensive measures of risk preference were also reviewed; the results of which are summarized in Table 2.

\section{Table 2 - Significance of Demographic Factors on Elicited Risk Preference}

\begin{tabular}{|c|c|c|c|c|c|c|c|c|c|c|}
\hline Study & Age & $\begin{array}{l}\text { Net } \\
\text { Wlth }\end{array}$ & Inc. & Edu. & Gndr & $\begin{array}{l}\text { Marital } \\
\text { Status }\end{array}$ & Race & $\begin{array}{l}\text { Self- } \\
\text { Empl. }\end{array}$ & Other & Measure \\
\hline $\begin{array}{l}\text { Chang et al. } \\
\text { (2004) }\end{array}$ & $\mathrm{X}^{* * *}$ & $x$ & & $\mathrm{X}^{* * *}$ & & & $\mathrm{X}^{* * *}$ & $\mathrm{X}^{*}$ & $\begin{array}{l}\text { Single woman; single } \\
\text { man; subjective risk } \\
\text { tolerance***; } \\
\text { employment status*** }\end{array}$ & $\begin{array}{l}\text { Risky assets } \\
\text { to net } \\
\text { worth }\end{array}$ \\
\hline $\begin{array}{l}\text { Halek \& } \\
\text { Eisenhauer, } \\
\text { (2001) }\end{array}$ & $\mathrm{X}^{* * *}$ & & & $x$ & $X^{* * *}$ & $\mathrm{X}^{* * *}$ & $x$ & $X^{* * *}$ & $\begin{array}{l}\text { Assets sq.***; gross } \\
\text { wlth.***; human } \\
\text { capital***/sq.***; } \\
\text { children; religion; } \\
\text { depression; drinker; } \\
\text { graduate; over } 65^{* * *} ; \\
\text { empl. status }\end{array}$ & $\begin{array}{l}\text { The Pratt- } \\
\text { Arrow } \\
\text { measure of } \\
\text { risk } \\
\text { aversion }\end{array}$ \\
\hline Grable, (2000) & $\mathrm{X}^{*}$ & & & $X^{*}$ & $\mathrm{X}^{*}$ & $\mathrm{X}^{*}$ & & & $\begin{array}{l}\text { Ecn. expectations*; } \\
\text { invest. experience*; } \\
\text { profnl. status* }\end{array}$ & $\begin{array}{l}20 \text {-item } \\
\text { assessment } \\
\text { instrument }\end{array}$ \\
\hline $\begin{array}{l}\text { Gibson et al. } \\
(2013)\end{array}$ & $\mathrm{X}^{* *}$ & $X^{* *}$ & $\mathrm{X}^{* *}$ & $\mathrm{X}^{*}$ & $\mathrm{X}^{* *}$ & $x$ & & & Financial advisor** & $\begin{array}{l}\text { FinaMetrica } \\
\text { risk } \\
\text { tolerance }\end{array}$ \\
\hline $\begin{array}{l}\text { Hallahan et al. } \\
\text { (2004) }\end{array}$ & $x$ & $\mathrm{X}^{* * *}$ & $\mathrm{X}^{* * * *}$ & $\mathrm{X}^{* * *}$ & $\mathrm{X}^{* * *}$ & $\mathrm{X}^{* * * *}$ & & & $\begin{array}{l}\text { \# of dependents**; } \\
\text { comb. inc.***; age } \\
\text { sq. } .^{* * *}\end{array}$ & $\begin{array}{l}\text { ProQuest } \\
\text { risk } \\
\text { tolerance } \\
\text { score. }\end{array}$ \\
\hline $\begin{array}{l}\text { Grable \& Joo, } \\
(2004)\end{array}$ & $x$ & $\mathrm{x}^{* *}$ & $\mathrm{X}^{* * *}$ & $\mathrm{X}^{* *}$ & $\mathrm{x}$ & $\mathrm{X}^{* *}$ & $x$ & & $\begin{array}{l}\text { First born; youngest; } \\
\text { financial satisfy.; home } \\
\text { ownership; fin. } \\
\text { knowledge**; self- } \\
\text { esteem*; type A; } \\
\text { sensation seeking }\end{array}$ & $\begin{array}{l}\text { 5-item } \\
\text { assessment } \\
\text { instrument }\end{array}$ \\
\hline
\end{tabular}

*** Significant at the .1 percent level

** Significant at the 1 percent level

* Significant at the 5 percent level

Gibson, Michayluk \& Van de Venter (2013) found that age, net wealth, income and gender are significant determinants of risk preference elicited using a 25 -question psychometric risk assessment test. Hallahan, Faff \& McKenzie (2004) used the same psychometric risk assessment 
test and found that gender, number of dependents, marital status, income and wealth are all significant. Grable (2000) used a 20-question questionnaire to elicit financial risk tolerance which is defined as the maximum amount of uncertainty someone is willing to take when making financial decision. Their findings indicate that people who are older, male, married, professionals, and those that have more education, financial knowledge and greater economic expectations are more risk seeking than their counterparts. Other studies report significant gender differences in risk preference; finding that women are generally more risk averse (Fehr-Duda, de Gennaro \& Schubert, 2006; Croson \& Gneezy, 2009; Eckel \& Grossman, 2002; 2008). The results of the reviewed literature on stable determinants of risk preference led to the identification of 16 demographic factors of interest outlined below.

\section{Stable Determinants of Risk Preference}

1. Age [Discrete]

2. Education [Some high school, High school, Some college/University, Post-secondary degree or certificate, Post-graduate]

3. Experience investing $[\mathrm{Y} / \mathrm{N}]$

4. Employment status [Part-time, Full-time, Student, Retired, Not Employed]

5. Occupation [Self-employed, Working Professional, Other]

6. Yearly income [Continuous]

7. Combined income [Continuous]

8. Gross wealth [Continuous]

9. Net wealth [Continuous]

10. Years until retirement $[0-9,10-19,20+]$

11. Gender [Male, Female]

12. Race [Black, White, Hispanic, Asian, Other]

13. Marital Status [Married, Couple, Single]

14. Head of household [Y/N]

15. Number of dependents [Discrete]

16. Number of people in household [Discrete] 


\subsection{The Effect of Emotions on Risk Preference}

Despite a growing body of evidence suggesting that decisions are often and significantly influenced by emotions (Bechara et al. 1997; Shiv et al. 2005; Lo et al. 2005), most decision theories fail to consider them (Sanfey, 2007; Gintis, 2011). Two of the most influential pioneers in the field of decision science addressed the need to include the effects of emotion on decision making decades ago and only now are researchers starting to explore the role they play in the decision-making process. In his seminal paper, Daniel Ellsberg (1961) acknowledged the idea that expectations about a prospect could change hourly with mood, and Herbert Simon (1983) believed that all theories of human rationality are incomplete unless they account for the role of emotion.

According to Lerner et al. (2015), decision theorists and researchers are increasingly embracing the notion of emotional decision making. Their findings show that the proportion of decision-making papers making reference to emotions, affect or mood has been increasing exponentially since 2010 with close to 450 papers published in 2013. Figure 2 presents a graphical representation of this finding.

Figure 2 - Number and Proportion of Articles Referencing Emotion, Affect and/or Mood

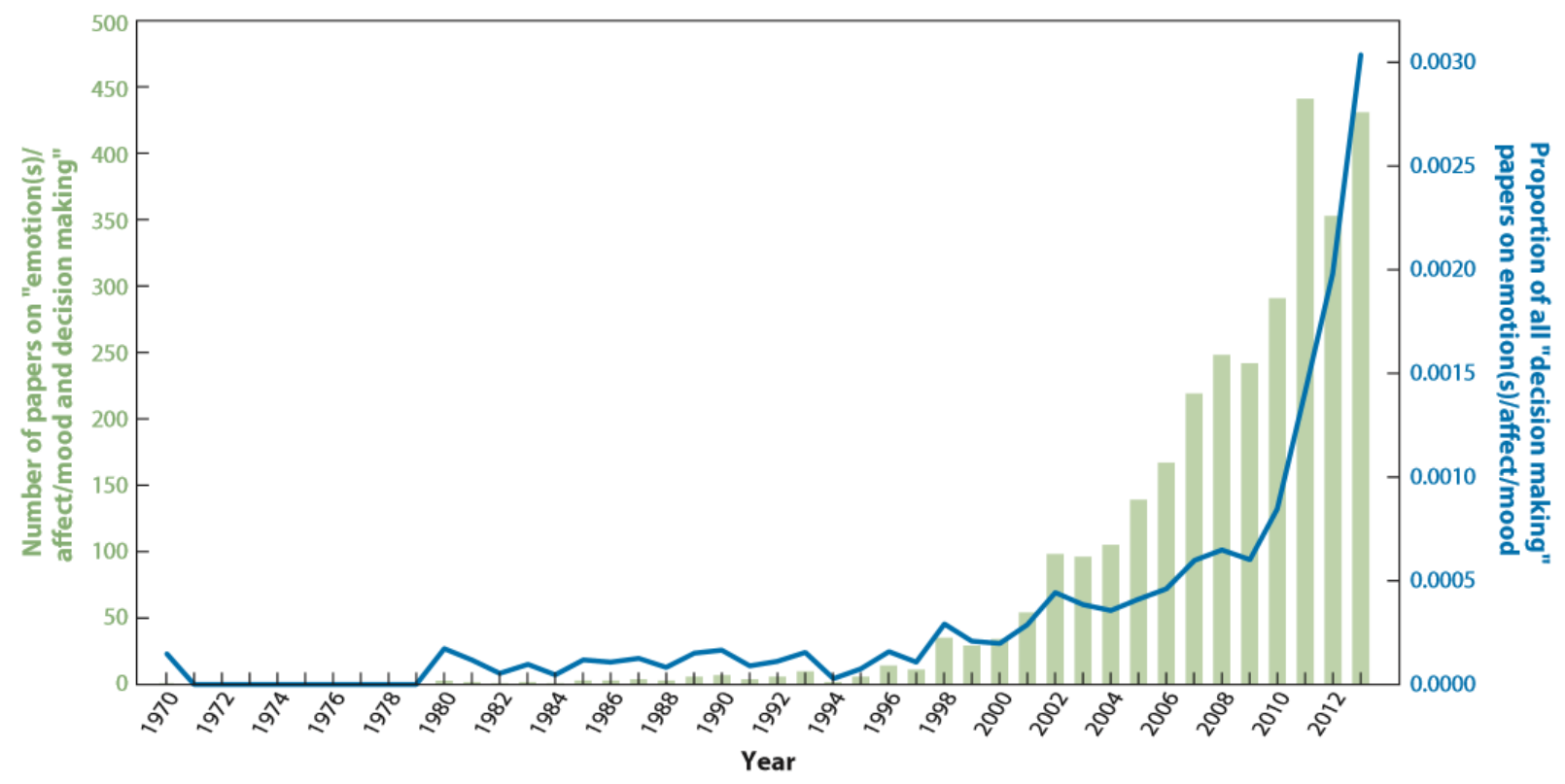

Source: Lerner et al. 2015 
Several theories have been developed to model the effect of emotion on decisions within the context of lottery-style gambles similar to the SG methods previously discussed. Some of the more prominent theories include regret theory (Bell, 1982; Loomes and Sugden, 1982; 1987), theory of disappointment (Bell, 1985; Loomes \& Sugden, 1986), and disappointment aversion (Gul, 1991). These theories attempt to describe the effect of integral (anticipated) emotions induced by the decision at hand. Incidental (carryover) emotions, on the other hand, are somewhat more elusive and inherently unpredictable. They are the emotions that occur as a result of activities unrelated to the decision at hand. Decision affect theory (Mellers et al. 1997) can account for both integral and incidental emotions in decision making but proposes the treatment of emotion as distinct from cognitive limitations.

The treatment of emotions and cognitive limitations has since been contested in studies showing that both are important considerations that are best unified into a single theory. Litt et al. (2008) provide strong evidence for this in showing that cognitive and affective processes interact through neurological systems observed in brain activity which can explain the central tenets of both decision affect theory and prospect theory. One objective of the present study is to acknowledge the interconnectedness of emotions and cognitive mechanisms by applying a series of transformation functions to a cognitive theory of choice preference in accordance with the effect that visceral factors have on choice preferences.

\subsection{Approaches to Measuring Affective States}

There are two general approaches to measuring affective states, the dimensional approach and the distinct-states approach. The dimensional approach seeks to model emotions based on a limited number of underlying dimensions. One example of the dimensional approach proposed by Ekkekakis (2012) measures core affect along three continuums: pleasure $\&$ displeasure, tension $\&$ relaxation, and energy \& tiredness. The distinct-states approach, on the other hand, treats each emotion as a discrete state. This approach allows emotions classified within the same dimension under the dimensional approach, such as anger and fear, to have different effects on behaviour (Lerner \& Keltner, 2000). While studies have explored the effect of incidental emotions on decisions, many differentiate only between positive and negative valences (Lerner $\&$ Keltner, 2000). The results of these studies were largely inconsistent and led to the emergence of two opposing theories, each with a body of evidence to support their claims - the mood 
maintenance hypothesis developed by Isen and Patrick (1983) and the affect infusion model developed by Forgas (1995). Recent studies show the effect of distinct emotions on risk preference differ in essential ways suggesting that incidental emotions should be decomposed into distinct states when exploring their effect on risk preference.

\subsubsection{Dimensional Approach}

Many of the earlier studies exploring the effect of incidental emotions on decisions adopted a two-track valence-based approach (Leith \& Baumeister, 1996; Kliger \& Levy, 2003; Grable \& Roszkowski, 2008; Kim \& Kanfer, 2009; Fehr-Duda et al. 2011). These studies demonstrated systematic tendencies associated with valences which led to the emergence of two opposing theories. The mood maintenance hypothesis (Isen \& Patrick, 1983) maintains that positive affective states will cause people to be more risk averse while negative states cause people to be more risk seeking. The affect infusion model (Forgas, 1995), on the other hand, claims the opposite effect (Finucane et al. 2000; Grable and Roszkowski, 2008; Leith and Baumeister, 1996). To date, the research is decidedly mixed; there is substantial evidence to support both frameworks.

Valence-based research and other dimensional approaches helped progress the study of decision making by demonstrating that affective states play a role in decision making, thereby challenging the assumption that risk preferences are stable over time. They are, however, limited to the analysis of positive and negative valences and provide little insight into the nature of the impact of specific emotions on thought processes. Furthermore, they assume that emotions of the same valence affect decisions similarly despite evidence that they often differ in essential ways (Smith \& Ellsworth, 1985; Lerner \& Keltner, 2000).

One earlier study clearly illustrates the need to characterize emotions as distinct states by showing that two emotions of the same valence, fear and anger, have opposing effects on risk preference (Kugler, Connolly \& Ordóñez, 2012). This study was restricted to the gain domain with no possibility of loss and found that participants primed with fear were significantly more risk averse than the angry group. While it did not include a control group as a baseline, the study substantiated concerns that the effect of emotions on risk preference must go beyond the valencebased approach. The likelihood that emotions of the same valence may have different or 
opposing effects on decision making presents a weakness in valence-based studies that is adequately addressed by adopting a distinct states approach to characterizing emotion.

\subsubsection{Distinct-States Approach}

Guiso, Sapienza \& Zingales (2013) took the study of affective states on decision making a step further by comparing the effect of fear on risk preference to changes in investor behaviour following the financial crash. The experiment showed that participants primed with fear had significantly lower certainty equivalents than those not exposed to the psychological prime in the gain domain, indicating greater aversion to risk. This study shows that risk preferences change over time in response to significant events and are influenced by affective states as well as provides evidence for the fear-based model in explaining stock market fluctuations.

In 2016, Treffers, Koellinger \& Picot used film clips to induce feelings of sadness, fear and joy in a group of students to explore the effect of emotion on a measure of risk preference elicited using the Multiple Price List method (Holt \& Laury, 2002). The experiment used three financial treatment groups: a fixed-stakes treatment group, that receive a flat fee for participating, as well as low- and high-stakes treatment groups that received the outcome of the gambles with a condition that participants cannot incur losses. The results showed no significant effects resulting from fearful or joyous states, however there was some evidence of increased risk aversion in participants experiencing sadness and that the magnitude of the financial stakes moderated the effect of mood. Treffers, Koellinger \& Picot note several reasons why their results may differ from previous studies, but ultimately advise caution in drawing premature conclusions from such experiments.

In the same year, Conte, Levati \& Nardi (2016) used film clips to induce joviality, sadness, fear and anger in undergraduate students and compared their risk preference to that of a control group not subjected to a psychological prime. The elicitation method used in this study is consistent with Hey (2001) and participants were financially compensated by way of a random lottery incentive mechanism that played out randomly selected lotteries at the end of the experiment. The results of the experiment revealed that all induced emotions significantly reduced aversion to risk and that joviality and fear have some effect on the probability weighting parameter assuming a Rank Dependent Expected Utility model. The effects of different emotions 
were also all found to be significantly different from one another with the exception of joviality $\&$ sadness and fear \& anger.

\subsubsection{Distinct-States Approach Assuming CPT}

While the results of experiments measuring the effect of distinct states on risk preference are promising, like their valence-based predecessors, they are largely inconclusive and must be further unpacked to discover the source of their inconsistencies. Empirical studies have shown that the measures of risk aversion used in these studies are too general and that risk preference is most accurately decomposed into the three distinct elements that characterize CPT (Köbberling \& Wakker, 2005).

Of the reviewed studies, only two explored the effect of emotion on the parameters that characterize risk preference under CPT. Callen et al. (2014) considered the effect of happiness, fear and exposure to violence in war-torn Afghanistan and Campos-Vazquez \& Cuilty (2014) considered the effect of anger, sadness and fear using similar methods to observe differences in model parameters resulting from an induced emotional state. Though they are not directly comparable, both studies demonstrate that distinct emotional states have different effects on risk preference.

The results presented by Callen et al. (2014) did not show any difference between neutral or happy states in the gain domain nor did they find exposure to violence alone to be significant. They did, however, discover limited effects of fear on risk preference and that the interaction of fear and exposure to violence resulted in a 16 percent larger certainty equivalent, which illustrates the exacerbating effect of recent trauma and fear on decision making. This study not only helps substantiate the effect of emotion on risk preference, but also builds on a growing body of literature demonstrating the effect of significant events on decision making (Eckel et al. 2009; Cameron \& Shah, 2015; Voors et al. 2012; Andrade et al. 2016).

Another study published in 2014 examines the effect of anger, sadness and fear on decision making in students studying economics in Mexico (Campos-Vazquez \& Cuilty, 2014). Participants were asked to self-assess as being either angry, sad or fearful after writing how they felt about shocking statistics concerning the ongoing violence or financial stability of Mexico. They then completed a questionnaire originally developed by Tanaka, Camerer, and Nguyen 
(2010) to elicit the parameters that characterize risk preference under CPT. The purpose of the study is to observe differences in decision weights as well as risk and loss aversion resulting from an induced emotional state and is the first to measure the effect of specific emotions assuming CPT. The results of this study showed that participants were more risk averse in gains and more risk seeking in losses when sad and roughly 50\% less loss averse when angry. Contrary to Callen et al. (2014), Campos-Vazquez \& Cuilty, (2014) did not obtain any significant results for fear. Participants in this study were not financially compensated, however, the results of the parameters were consistent with the literature.

\subsection{The Effect of Other Visceral Factors on Risk Preference}

Visceral factors include drive states such as hunger, thirst, sexual desire, drug cravings, physical pain, as well as moods and emotions and can influence the value people place on goods and actions. Depending on the intensity of these desires, visceral factors can magnify the anticipated rewards of a good or action and focus on the goals associated with the current state to the exclusion of all others (Loewenstein, 1996). Studies examining other visceral factors have found significant effects of factors such as hunger (Symmonds et al. 2010), cognitive fatigue (Hockey et al. 2000), sleep deprivation (Killgore et al. 2006), and stress (Buckert et al. 2014) on risk preference elicited using lottery-style decision tasks.

In addition to incidental (carryover) emotions, the current study seeks to understand the effect of other visceral factors shown to influence risk preference both directly and indirectly through their interaction with other determinants of risk preference. In addition to affective states, other visceral factors under consideration include hunger, sleep deprivation and stress, all of which have been shown to interact with one another and with distinct affective states.

\subsubsection{Hunger}

Hunger has long been studied in both humans and animals and can be measured by the subject's metabolic state (Barnard \& Brown, 1985; Carr, 1996; Figlewicz, Naleid \& Sipols, 2007).

According to Symmonds et al. (2010) hormones circulating through the body report the status of energy reserves to regions of the brain strongly implicated in risk and reward-based decision making. The physiological connection in addition to observed risky feeding patterns in animals when below a metabolic reference point prompted their study of the effect of metabolic state on financial risk-taking. Participants in this study fasted 14 hours prior to each session and were fed 
a large meal one hour into the three-hour session. Throughout each session, chemical identifiers of hunger and satiation in the blood were monitored periodically along with a self-assessed visual analogue score. Risk preference was measured throughout the experiment according to the percentage of risky lotteries selected in a series of 200 paired lottery-style gambles. The results of the experiment show a significant increase in risky choices and a decrease in risk premium immediately after eating, and increased aversion to risk relative to the fasted state one hour after eating. This study shows a quantifiable and systematic link between metabolic state and risk preference in humans with direct implications on financial decisions.

\subsubsection{Sleep Deprivation}

Sleep deprivation (SD) is highly relevant to today's work environment and has been studied extensively within the context of professional decision making, but there is little work exploring its effect on broader human functioning. Some studies relate SD to mood, diminished innovative thinking, poor planning, frequent changes to strategies and susceptibility to auditory and visual distractions (Pilcher \& Huffcutt, 1996; Mander et al. 2008). It has also been proposed that people suffering from SD will be less able to appreciate difficult and dynamic situations, assess risk, anticipate consequences, control mood, as well as experience diminished memory of past experiences and personal insight (Harrison \& Horne, 2000).

Previous experiments showing rule-based convergent skills to be relatively unaffected by SD led to theories that high-level complex skills such as decision making under risk were less affected than basic skills like reaction time or memory. Harrison \& Horne (2000) conducted an extensive literature review of the effect of SD on decision making. In it, they challenge the theory that high-level complex skills are unaffected by SD on the basis that real world applications of these skills often require both convergent and divergent thinking and the latter rely on the prefrontal cortex which studies show is particularly affected by SD and is among the first brain regions to be affected. Thomas et al. (2000), for example, found SD to most significantly affect the thalamus and prefrontal cortex regions of the brain which are responsible for alertness, attention and higher-order cognitive processes. In light of these findings, Harrison $\&$ Horne (2000) propose that people suffering from SD will be less able to perform the tasks that rely on the functional integrity of brain regions most greatly affected by SD. These involve, 
among others, appreciating difficult and dynamic situations, assessing risk, anticipating consequences, controlling mood, memory of past occurrences and personal insight.

While there are few studies exploring the direct effects of SD on decision making under risk, many of the findings linking SD with diminished divergent skills directly influence risk preference through its effect on the brain region that governs higher-order cognitive functions. Killgore et al. (2006) observed a diminished capacity to weigh short-term gains against larger long-term losses in sleep deprived participants. These results are similar to that of another Iowa gambling task experiment examining the behaviour of patients who suffer from lesions to their ventromedial prefrontal cortex (Bechara et al. 1997). This experiment shows how SD can lead to risk seeking behavior through the functional capacity of the prefrontal cortex. One notable limitation of this study and the use of the Iowa gambling task to elicit risk preferences is that it does not differentiate between gains and losses.

Two SD studies designed to differentiate between gains and losses find similar effects of SD in the gain domain. McKenna, Dickinson, Orff, \& Drummond (2007) conducted an experiment measuring the effect of SD on risk and ambiguity preference in both the gain and loss domain using lottery-style decision tasks. While this study did not find that SD affected ambiguity aversion as hypothesized, the sleep deprived group were significantly less risk averse in gains and less risk seeking in losses indicating that SD acts to moderate risk preference. Another study differentiating between gains and losses was designed to explore the effect of SD on neural responses to decision making (Venkatraman et al. 2007). Based on their observations of brain activity while completing gambling tasks Venkatraman et al. (2007) find that SD can lead to decreased aversion to risk and a diminished ability to learn from the negative consequences of risky decisions. This is consistent with the results of McKenna et al. (2007). Interestingly, Venkatraman et al. (2007) also show that SD acts to modulate activation in two areas of the brain associated with risky decision making and emotional processing, which supports the interconnectedness of emotions and risk preference.

\subsubsection{Stress}

According to the American Psychological Association there are three types of stress: acute stress, episodic acute stress and chronic stress; all three of which have been shown to cause psychological and neuropsychological reactions linked to cognitive, emotional, physiological 
and behavioural responses. Acute stress occurs in response to pressures concerning the recent past and near future and can be induced by introducing time pressures, physiological stressors (Porcelli \& Delgado, 2009) or psychosocial stressors (Buckert et al. 2014). In light of concerns that different types of stressors will trigger different neurological reactions and lead to different responses, the current study concerns only psychosocial stressors.

Buckert, Schwieren, Kudielka \& Fiebach (2014) used lottery-style gambles in gain, loss and mixed domains to assess the effect of a psychosocial stressor (TSST-G) on risk and ambiguity preferences in a group of students in Germany. Several measures of mood were also measured subjectively along three dimensions before and after the stressor was introduced. The results of the experiment indicate that the stress group were significantly more risk seeking in the gain domain, but no other differences relating to risk or ambiguity preference were discovered.

Another study using the same stress induction procedure and measured mood along similar dimensions was conducted by Cahlíková \& Cingl (2017). After controlling for the 'Big Five' personality traits (Goldberg et al. 2006) the results of this study indicate that men are significantly more risk averse when stressed. The effects for women are similar, but insignificant.

Bendahan et al. (2017) explored the effect of acute stress on risk preference as a time dependent factor by comparing the risk preferences of the stress and control groups at different points in time following the stress induction. The results of the study indicate that participants exposed to the stressor were significantly more risk seeking immediately following the stress onset. This effect gradually faded as participants became increasingly risk averse over time with the effects of stress reaching insignificance after approximately 45 minutes.

\subsection{Visceral Factor Interactions}

This growing body of knowledge is producing mounting evidence that affective states not only interact with one another, but with other visceral factors that have not been thoroughly explored in combination with affective states (Uhart et al. 2006; Raspopow et al. 2010; Mikolajczak et al. 2007; 2008). The possibility of interaction among all the above listed visceral factors highlights the importance of assessing the impact of multiple factors simultaneously. 
Affective states, for example, have distinct effects that can moderate or exacerbate the effects of other states (Kugler et al. 2012) and visceral factors (Hockey et al. 2000; Buckert et al. 2014). The effects of sleep deprivation can also impact emotional and higher-order cognitive processes (Pilcher \& Huffcutt, 1996; Dinges et al. 1997) and have been shown to be gender-sensitive (Ferrara et al. 2015) and increase with age (Killgore et al. 2006). Stress has been linked to physiological, emotional and cognitive changes indicating interactions between multiple facets governing human behaviour including affective states (Uhart et al. 2006; Raspopow et al. 2010; Mikolajczak et al. 2007; 2008) and personality traits (Campbell \& Ehlert, 2012). On this last point, it is recommended that all studies examining the effect of stress on behaviour perform psychometric assessments to address relevant personality traits (Campbell \& Ehlert, 2012).

\subsection{Self-Assessed Measures of Visceral Factors}

Self-assessment methods have shown to be a valid and reliable method for assessing subjective factors in a variety of contexts and are generally considered an important source of information (Mistar, 2011; Ross, 2006). Several of the reviewed studies reported finding significant effects on risk preference associated with self-assessed measures of distinct affective states (CamposVazquez \& Cuilty, 2014; Fehr-Duda et al. 2011; Hockey et al. 2000; Kugler, Connolly \& Ordóñez, 2012; Cahlíková \& Cingl, 2017), hunger (Symmonds et al. 2010), cognitive fatigue (Hockey et al. 2000; Lim et al. 2010), arousal (Buckert et al. 2014) and stress (Hoeger Bement et al. 2010; Kern et al. 2008; Kirschbaum et al. 1995; Simeon et al. 2007).

In addition to using self-assessed measures of visceral factors, many of the reviewed studies measure physiological indicators and found significant correlations between the two (Uhart et al. 2006; Raspopow et al. 2010; Mikolajczak et al. 2007; 2008). The two most common selfassessment methods in the literature are the Likert scale and the visual analogue scale. Although the two methods are comparable (Jaeschke et al. 1990; Kuhlmann et al. 2017), visual analogue scales were selected for this study because the majority of the reviewed studies focusing on the factors of interest and all studies showing strong correlations between physiological indicators and self-assessment methods used visual analogue scales to identify the presence and intensity of visceral factors (Hockey et al. 2000; Symmonds et al. 2010; Mullette-Gillman et al. 2015; Lim et al. 2010). 


\section{CHAPTER 4 \\ THEORY SELECTION}

The theory selection process for this study involved an exploration of the systematic effects in human decision making to determine a set of minimum criteria for a theory to be capable of describing the decision-making process. Each of the four minimum considerations are listed and described below. While a detailed account of the theories considered is beyond the scope of this thesis, several EU-generalizations and homeomorphic theories were explored before deciding on CPT (Tversky \& Kahneman, 1992). Other factors considered in the selection of this theory include the available elicitation methods that make this theory completely observable using nonparametric methods (Abdellaoui et al. 2016) and the general consensus amongst practitioners promoting CPT as the new standard in descriptive theories of choice preference (Starmer, 2000; Kothiyal et al. 2014; Camerer, 1998).

\subsection{Minimum Considerations}

\subsubsection{Nonlinear Treatment of Outcomes and Probabilities}

The fourfold pattern of risk preference discovered in outcomes (Markowitz, 1952) and probabilities (Tversky \& Kahneman, 1992) cannot be explained by a univariate model and necessitates the nonlinear transformation of both outcomes and probabilities. Markowitz (1952) first identified a fourfold pattern in decision making under risk when examining the magnitude of outcomes which was later confirmed by Hershey and Schoemaker (1980) using a magnified lottery system. These studies demonstrated that people are generally risk averse in gains and risk seeking in losses when outcomes are large, and risk seeking in gains and risk averse in losses when outcomes are small.

A similar pattern was later discovered by Tversky and Kahneman (1992) who found that people tend to react similarly when confronted with different degrees of probability. They showed that people are also risk averse when confronted with large probability of a gain or small probability of a loss and risk seeking when confronted with a large probability of a loss or a small probability of a gain. Mallpress et al. (2015) later examined the fourfold pattern in outcome probabilities from an evolutionary perspective and found that, in certain environments, the fourfold pattern can be seen to maximize lifetime reproductive success and is the likely result 
of evolutionary adaptation. Though Tversky and Kahneman did not address Markowitz's original fourfold pattern in either prospect theory or CPT, a later study conducted by Scholten and Read (2014) extended CPT to account for the fourfold pattern in outcome magnitudes by combining the theory's increasingly elastic probability-weighting function with a decreasingly elastic utility function.

In addition to the fourfold patterns of risk preference, it is also shown that observed violations of EU due to the certainty effect and boundary effects, such as Maurice Allais' common consequence and ratio effects, are most readily and logically explained by the nonlinear transformation of probabilities (Schmidt, 2000).

\subsubsection{Reference Points}

Another pervasive effect in decision making is the tendency for people to treat losses differently than gains. Framing effects refer to a tendency for people to interpret information differently depending on whether it is framed as a gain or as a loss (Tversky \& Kahneman 1981; 1986; Kahneman \& Tversky, 1984). The reference point is the point that distinguishes between what someone considers a gain and a loss. This is a key feature in modern descriptive theories of choice preference that contests a fundamental assumption in many theories that utility is solely a function of cumulative wealth. Instead, these theories contend that people code and evaluate outcomes based on deviations from a reference point.

While there is little debate as to the existence of a reference point, there is no reliable and objective way to determine an individual's reference point. The reference point is typically considered to be the status quo; or rather the point of no gain and no loss. Studies have, however, shown that the reference point can lie either in the gain or loss regions of the utility function (Fishburn \& Kochenburger, 1979; Zhang et al. 2016). At times, the reference point can coincide with a goal (Heath, Larrick \& Wu, 1999) and has also been shown to change after each decision in a sequence of gambles (Barkan \& Busemeyer, 2003). One common example of a reference point that does not coincide with the status quo is referred to as the house money effect (Thaler $\&$ Johnson, 1990). This effect describes the willingness of people to engage in riskier behaviour with profits from a recent gain. So long as the loss is smaller than the recent gain people tend to integrate the outcomes and code the loss as a reduction of gains rather than a loss. 
By modeling decision making in relation to deviations from a reference point we can also account for loss aversion. Loss aversion refers to a general tendency in decision making where decision makers consider losses more heavily than equivalent gains (Binswanger, 1981; Hardie et al. 1993; Cohen et al. 1987; Wakker, 2010). The existence of loss aversion implies a need to establish a reference point when modelling choice preferences and limits the available theories to those that allow gains and losses to be treated differently. Loss aversion is explained in more detail in section 4.3.3.

\subsubsection{Monotonicity}

It is often claimed that more is always better and while few would disagree when evaluating riskless choices, the monotonicity of preferences necessarily carries over to the evaluation of risky choices. Monotonicity, or rather first-order stochastic dominance, describes a choice between a set of gambles where one strictly dominates all others and has proven one of the more controversial assumptions in recent history. Violations of monotonicity occur when an increase in the value of one of the outcomes in a prospect, holding constant all other criteria, causes a decrease in the value of the prospect (or vice versa).

Despite its normative appeal and usefulness in unifying certain and risky prospects in utility analysis, many empirical studies researching specific types of lottery questions have demonstrated systematic violations of monotonicity (Birnbaum \& Navarrete, 1998). When models that adhere to monotonicity are tested under these conditions they tend to underperform relative to models that predict violations of monotonicity (Birnbaum, 2005). In this sense, the assumption of monotonicity acts as a constraint on the model, but one that is fundamental to the mathematization of choice preferences by ensuring continuity and the uniqueness of indifference points (Farquhar, 1984). It is for this reason as well as its normative appeal that academics have been so opposed to models that predict violations of monotonicity despite empirical evidence suggesting that people systematically violate it under certain conditions.

\subsection{Cumulative Prospect Theory}

Cumulative prospect theory (CPT) is a popular cognitive decision theory capable of handling prospects with any number of outcomes with known or unknown probabilities. This theory accounts for all the conditions considered to be minimal requirements for a descriptive theory of choice leading several prominent figures in mathematical psychology and behavioural economics 
to suggest that it replace EU as the new standard for descriptive theories of choice preference (Starmer, 2000; Kothiyal et al. 2014; Camerer, 1998).

The origins of CPT date back to the development of original prospect theory (OPT) which was introduced by Daniel Kahneman and Amos Tversky as an alternative to the axiom-based approaches to modeling preferences that made little effort to describe the underlying mental processes driving decision making (Kahneman \& Tversky, 1979). OPT deviates substantially from EU and EU-generalizations in its evaluation of prospects according to changes in wealth rather than final states of wealth, the different treatment of gains versus losses and its introduction of decision weights. It was the first widely accepted decision theory that abandoned the conventional approach in favour of a procedural approach and ushered in an era of homeomorphic theories that abandoned principles of rationality in favour of principles founded in psychology.

In constructing this theory, Kahneman and Tversky drew from the mounting evidence in psychology demonstrating that the effects of human cognition produce systematic biases and heuristics in decision making under risk. OPT contends that the prospects are first edited according to several operations in the editing phase prior to being evaluated in the evaluation phase. Once prospects are edited, they are evaluated according to the subjective evaluation of both probabilities and outcomes. This is consistent with empirical evidence supporting the existence of cognitive mechanisms that simplify decision tasks when people are confronted with unmanageably large amounts of information.

Shortly after its release, OPT came under criticism for violating first-order stochastic dominance, often referred to as monotonicity. Kahneman and Tversky defend their theory by claiming that most of these violations are edited out in the editing phase and that violations of monotonicity do occur. Largely in response to these criticisms, Tversky and Kahneman (1992) developed CPT as an extension of OPT capable of handling any number of outcomes with known or unknown probabilities. CPT is the sum of two rank-dependent expected utility models and, as such, does not require an editing operation to avoid violations of first-order stochastic dominance. The new theory accounts for framing effects, nonlinear preferences, source dependence, risk seeking and loss aversion, all of which have been shown to be systematic and are considered minimal requirements for a descriptive theory of choice. Though Tversky and 
Kahneman (1992) admit they sacrificed some minor features of OPT in the formulation of this new theory, CPT follows a more conventional approach, is applicable to a broader range of situations and provides a convenient representation of decision weights. CPT is described as follows (Schmidt, 2002; Köbberling \& Wakker, 2005),

$$
V(P)=\sum_{i=1}^{n} \pi_{i} u\left(X_{i}\right)
$$

where $V(P)$ is the value of prospect $P, \pi_{i}$ is the decision weight associated with outcome $X_{i}$ and $u$ is the utility function that describes the utility associated with outcome $x_{i}$. The utility function is characterized as continuous and non-decreasing with $u(0)=0$.

For n-outcome gambles, $\left(p_{1}, X_{1} ; \ldots ; p_{n}, X_{n}\right)$ with $X_{1} \geq \cdots \geq X_{k} \geq 0>X_{k+1} \geq X_{n}$, the decision weights are described as follows,

$$
\pi_{i}= \begin{cases}w^{+}\left(\sum_{j=1}^{i} p_{j}\right)-w^{+}\left(\sum_{j=1}^{i-1} p_{j}\right), & \text { if } i \leq k \\ w^{-}\left(\sum_{j=i}^{n} p_{j}\right)-w^{-}\left(\sum_{j=i+1}^{n} p_{j}\right), & \text { if } i>k\end{cases}
$$

where $w^{+}$and $w^{-}$are the probability weighting functions for gains and losses, respectively, $\sum_{j=1}^{i} p_{j}$ is the sum of all probabilities associated with positive outcomes up to and including $X_{i}$, and $\sum_{j=i}^{n} p_{j}$ is the sum of all probabilities associated with negative outcomes up to and including $X_{n}$. The decision weights are generated by the probability weighting function over the interval $[0,1]$ such that they are continuous and non-decreasing with $w^{+}(0)=w^{-}(0)=0$ and $w^{+}(1)=$ $w^{-}(1)=1$. This is consistent with both Quiggin (1981) and Schmeidler's (1989) characterization of rank dependent expected utility theory and ensures the adherence to firstorder stochastic dominance. 
Drawing on a previous example depicted in Figure 1, the same preference relation assuming CPT implies:

$$
\begin{gathered}
w^{+}(1) \cdot u(\$ 1 M)>w^{+}(.1) \cdot u(\$ 5 M)+\left[w^{+}(.99)-w^{+}(.1)\right] \cdot u(\$ 1 M)+\left[1-w^{+}(.99)\right] \cdot u(\$ 0) \\
=\left[1-\left(w^{+}(.99)-w^{+}(.1)\right)\right] \cdot u(\$ 1 M)>w^{+}(.1) \cdot u(\$ 5 M) \\
\& \\
w^{+}(.1) \cdot u(\$ 5 M)+\left[1-w^{+}(.1)\right] \cdot u(\$ 0)>w^{+}(.11) \cdot u(\$ 1 M)+\left[1-w^{+}(.11)\right] \cdot u(\$ 0) \\
=w^{+}(.1) \cdot u(\$ 5 M)>w^{+}(.11) \cdot u(\$ 1 M)
\end{gathered}
$$

Since CPT allows for the non-linear transformation of probabilities, the value of [1 $\left.w^{+}(.99)+w^{+}(.1)\right]$ can be greater than $w^{+}(.11)$ to account for the observed preferences that violate the independence axiom under EU. This implies that the decision weight placed on a certain outcome of $\$ 1 \mathrm{M}$ minus the decision weight placed on an $89 \%$ chance of obtaining $\$ 1 \mathrm{M}$ in question (a) is greater than the decision weight placed on an $11 \%$ chance of obtaining $\$ 1 \mathrm{M}$ in question (b).

This study considers only the special case where prospects are comprised of two mutually exclusive and collectively exhaustive risky outcomes, $\left(X_{1}, p, X_{2}\right)$ with $X_{1}>X_{2} \geq 0$. Assuming CPT, this implies:

$$
w(p) u\left(X_{1}\right)+[1-w(p)] u\left(X_{2}\right)
$$

\subsection{Parametric Specification}

Risk preference has been shown to be most accurately decomposed into the three distinct elements that characterize CPT: basic utility, probability weighting, and loss aversion (Köbberling \& Wakker, 2005). Basic utility describes participants' subjective interpretation of outcomes, decision weights characterize their subjective interpretation of explicitly stated probabilities and loss aversion is a measure of how strongly they feel about a loss relative to a gain of the same magnitude.

This study is concerned with the effect of all three elements across the gain, loss and mixed domains. The utility function is defined by the power parameter, the probability weighting function is defined by the two-parameter specification presented by Gonzalez \& Wu (1999) and 
loss aversion is defined by an index presented by Booij (2009) that closely resembles Kahneman \& Tversky's (1979) original specification. All parameters in these elements are unique, separable and have independent psychological interpretations.

\subsubsection{Power Parameter}

The utility function is defined by the most popular single-parameter parametric family of utility known as the power family or the family of constant relative risk aversion (CRRA). The reason for the popularity of this parametric family of utility is thought to be because of its simplicity and fit with empirical data (Wakker, 2004b; Wakker, 2008). For positive outcomes, this parametric family exhibits constant relative risk aversion and is not affected by unit changes. In other words, preferences between outcomes are not affected if both outcomes are multiplied by the same positive constant. The parametric specification of the utility function is described as follows (Wakker, 2010),

$$
\begin{aligned}
& \text { for } X>0 \\
& \qquad\left\{\begin{array}{rr}
\text { for } \theta>0, & u(X)=X^{\theta} \\
\text { for } \theta=0, & u(X)=\ln (X) \\
\text { for } \theta<0, & u(X)=-X^{\theta}
\end{array}\right\}
\end{aligned}
$$

where $u$ is the utility function, $X$ is a positive outcome and $\theta$ is the power parameter. Wakker (2008) argues that the transition from $\theta<0$ to $\theta>0$ is the only natural way to define the family. Graphical representations of the utility function are presented in Figure 3.

Figure 3 - Example of Utility Functions

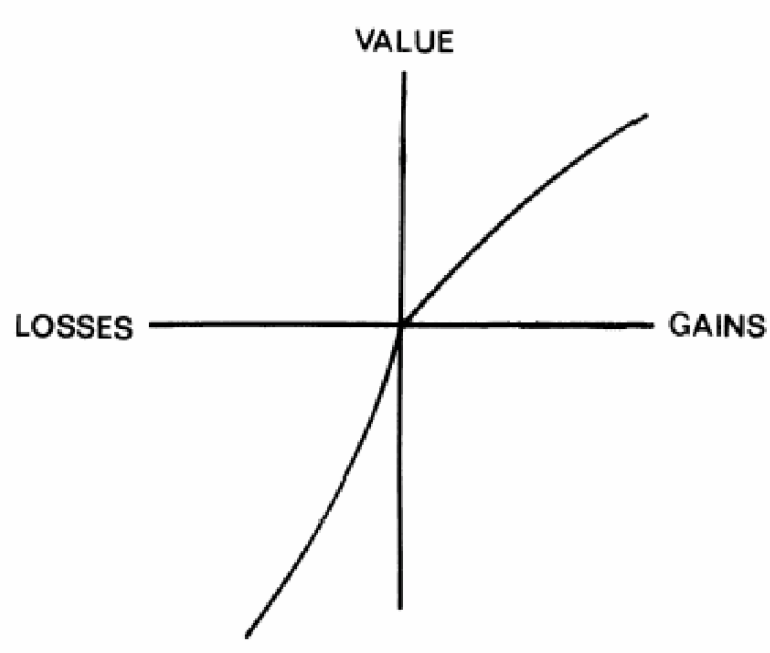

Source: Wu, G., Zhang, J. \& Gonzalez, R.

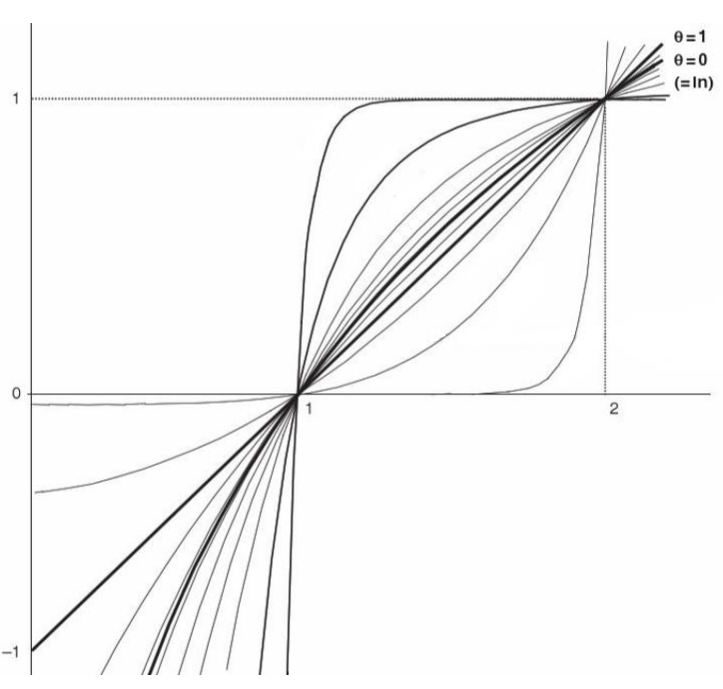

Source: Wakker. P. P. (2010). 


\subsubsection{Decision Weight Parameters}

There are many functional forms used in the literature to construct probability weighting functions. Single parameter forms are popular, but do not give the same degree of latitude as the two-parameter form in fitting the probability weighting function to the data. The single parameter forms such as that proposed by Kahneman and Tversky (1992) force the probability weighting function through linearity at a predefined point. Many of the participants in the study were either consistently over or underweighting probabilities and so forcing the function through linearity would not allow for an adequately representative function. Other two-parameter functionals such as that proposed by Prelec (1998), do not allow for full separation of the elevation and curvature which can bias inferences if they do not covary accordingly (Gonzalez \& Wu, 1999; Booij et al. 2009).

The function form used in this study to infer decision weights is a variation of that used by Goldstein and Einhorn (1987) referred to as the "linear in long odds". It is a two-parameter function used extensively in the literature to describe the shape of the probability weighting function (Gonzalez \& Wu, 1999; Abdellaoui et al. 2005; Lattimore et al. 1992). This form is particularly useful because it allows for changes in both the elevation and curvature of the function each of which have distinct psychological interpretations described below. Each of the parameters in this model are uniquely determined, vary the curvature and elevation of the probability weighting function separately, and independent of one another with the exception of fixed points at 0 and 1 (Gonzalez \& Wu, 1999). The parametric specification of the probability weighting function is described as follows,

$$
\left\{\begin{array}{l}
w^{+}(p)=\frac{\delta^{+} p^{\gamma^{+}}}{\delta^{+} p^{\gamma^{+}+(1-p)^{\gamma^{+}}}} \\
w^{-}(p)=\frac{\delta^{-} p^{\gamma^{-}}}{\delta^{-} p^{\gamma^{-}+(1-p)^{\gamma^{-}}}}
\end{array}\right\}
$$

The elevation of the probability weighting function is determined by the $\delta$ parameter. This parameter reflects the degree of over- or under-weighing of probabilities and determines the point at which the decision weight equals the objective probability, $p=w(p)$. According to Gonzalez \& Wu (1999), this parameter can be interpreted as the degree of attractiveness of gambling. If the function characterizes someone as having over-weighed (under-weighed) the 
explicitly stated probability of winning the large amount in the gain domain, they are said to be optimistic (pessimistic). Conversely, if they are characterized as having over-weighed (underweighed) the probability of losing the large amount in the loss domain they are said to be pessimistic (optimistic). Figure 4 illustrates the effect of changes in the elevation parameter, $\delta$, from .2 to 1.8 holding the curvature parameter, $\gamma$, constant at .6 .

\section{Figure 4 - Changes in the Elevation Parameter on the Probability Weighting Function}

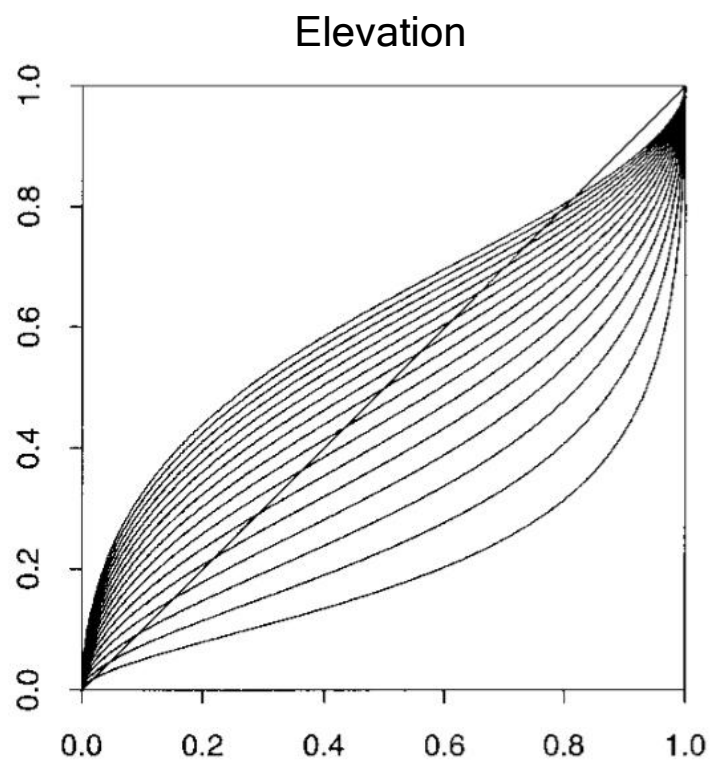

Source: Gonzalez, R. \& Wu, G. (1999).

The curvature of the probability weighting function is determined by the parameter $\gamma$ and is interpreted as the degree to which an individual discriminates between changes in probabilities across the interval $[0,1]$, where 0 implies an impossible outcome and 1 a certain outcome (Gonzalez \& Wu, 1999). In other words, this parameter captures the individual's sensitivity to changes in probability over the interval $[0,1]$. It has been found that people are more sensitive to changes in probabilities around 0 and 1 which indicates a steeper slope in the probability weighting function approaching these bounds and implies an inverse S-shaped weighting function. This shape implies that a change from $0 \%$ to $1 \%$ or from $99 \%$ to $100 \%$ generally have more impact than a change from $10 \%$ to $11 \%$. This is also consistent with the psychological principle of diminishing sensitivity if we consider 0 and 1 to be reference points in the decision weight space. The further from these points, the less impact changes will have on behaviour. The 
inverse S-shaped weighting function satisfies bounded subadditivity (both upper and lower subadditivity) and is characterized by a $\gamma$ value less than 1 (Tversky \& Fox, 1995). Figure 5 illustrates the effect of changes in the curvature parameter, $\gamma$, from .2 to 1.8 holding the elevation parameter, $\delta$, constant at .6 .

Figure 5 - Changes in the Curvature Parameter on the Probability Weighting Function

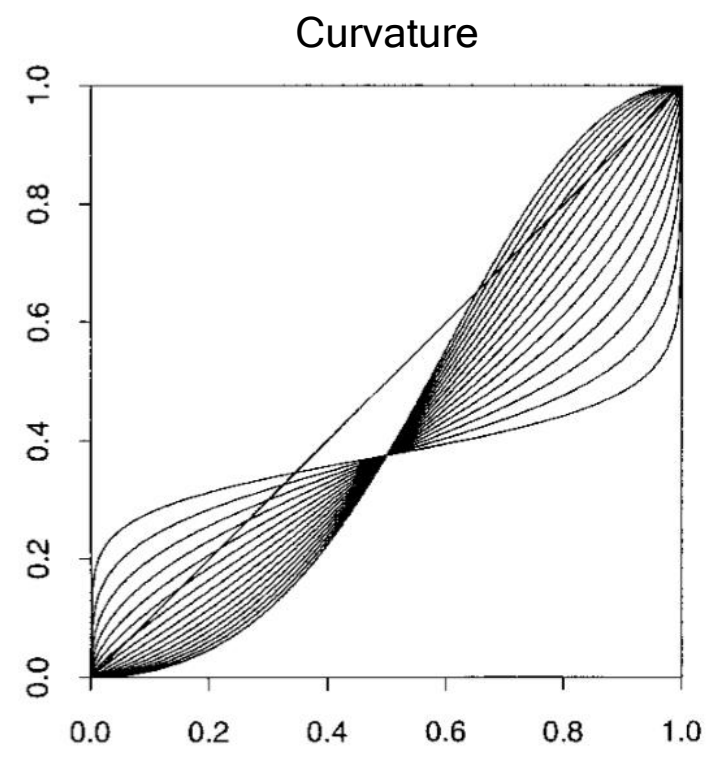

Source: Gonzalez, R. \& Wu, G. (1999).

The effects of each of these parameters on the probability weighting function can be described as follows:

- If $\delta=\gamma=1$ then $w(p)=p$ and CPT reduces to Expected Utility.

- If $\gamma=1$ and $\delta>1$, there is overweighting of probabilities everywhere in the interval $[0,1]$. In this context, this can be interpreted as optimistic in the gain domain and pessimistic in the loss domain.

- If $\gamma=1$ and $\delta<1$, there is underweighting of probabilities everywhere in the interval $[0,1]$ and an opposite interpretation can be inferred.

- If $\gamma \neq 1$, there is over and underweighting at different points over the interval $[0,1]$.

- If $\gamma>1$, the probability weighting function is $\mathrm{S}$-shaped which can be interpreted as underweighting of small probabilities and overweighting of large probabilities. 
- If $\gamma<1$, the probability weighting function is inverse S-shaped and can be interpreted as underweighting of large probabilities and overweighting small of probabilities.

- If $\gamma>1$, an increase in the parameter $\delta$ will decrease the point at which the explicitly stated probability equals the corresponding decision weight (the crossover point).

- If $\gamma<1$, an increase in the parameter $\delta$ will increase the crossover point.

Figure 6 presents a graphical depiction of probability weighting functions estimated using the linear in long odds form.

Figure 6 - Empirical Estimates of the Probability Weighting Function

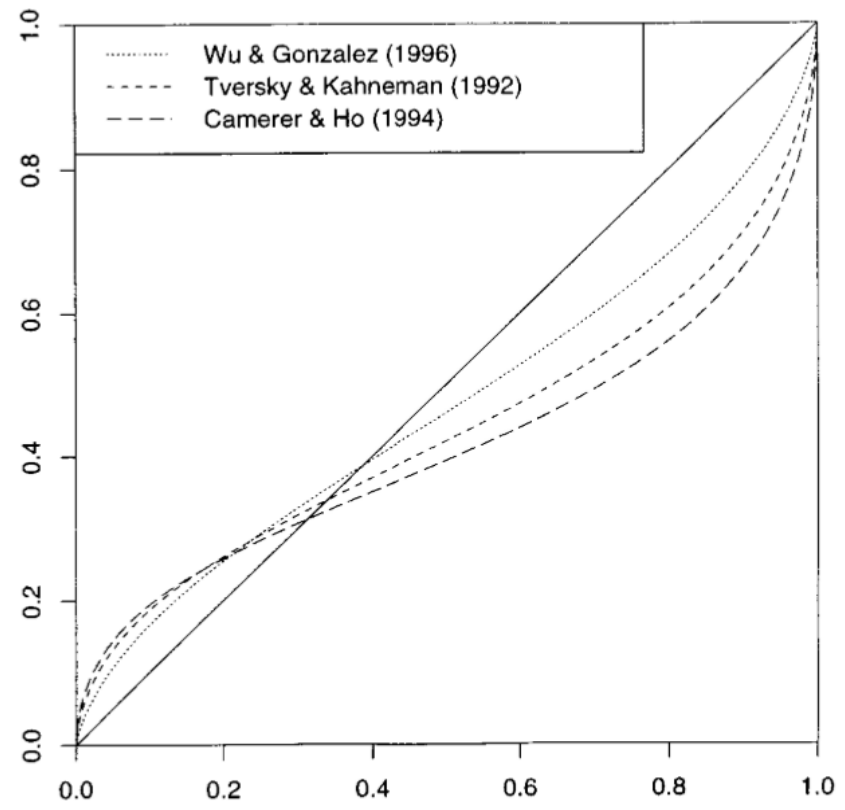

Source: Gonzalez, R. \& Wu, G. (1999).

\subsubsection{Loss Aversion Parameter}

Loss aversion was first hypothesized by Markowitz (1952) and more recently formalized by Kahneman \& Tversky (1979). It has been shown to be one of the more prominent effects in decision making capable of explaining field and experimental data in a variety of contexts. The concept of loss aversion has been used to explain the endowment effect (Thaler, 1980), status quo bias (Samuelson and Zeckhauser, 1988), the equity premium puzzle (Benartzi and Thaler, 1995), downward-sloping labour supply (Camerer \& Hogarth, 1999) and the overtime premium puzzle (Dunn, 1996). The growing importance of loss aversion in explaining anomalies in 
decision making has prompted leading experts to recommend that it be integrated into all relevant economic analyses (Rabin, 1998; Kahneman, Knetsch \& Thaler, 1991).

Loss aversion is a measure of the significance of a loss relative to an equivalent gain and acts to govern the exchange rate between gain and loss utility units. Loss aversion is therefore a product of utility units in the gain domain relative to utility units in the loss domain and has no meaning independent of utility. It is also important to note that there is no standard agreed upon definition of loss aversion and the magnitude of the loss aversion parameter depends on the specification used (Abdellaoui, Bleichrodt \& Paraschiv, 2007).

To define loss aversion, it is necessary to first determine an appropriate reference point. This study uses the status quo to distinguish between gains and losses and adopts a specification of loss aversion, $\lambda$, proposed by Booij (2009),

$$
\lambda=\frac{u\left(Y_{0}\right) X_{0}}{u\left(X_{0}\right) Y_{0}}
$$

where $X_{0}$ and $Y_{0}$ are the first positions in the standard sequence of utility units in the gain and loss domain, respectively. The values of $X_{0}$ and $Y_{0}$ are set by the researcher according to the range and magnitude of outcome being investigated. In this study, $X_{0}$ is set to $\$ 100$ and $Y_{0}$ is set to $-\$ 100$. Under this specification, individuals are considered loss averse if $\lambda>1$.

This specification is considered an approximation of the specification initially proposed by Benartzi and Thaler (1995) and later formalized by Köbberling and Wakker (2005) that closely resembles Kahneman \& Tversky's (1979) original specification. Since it is designed to measure the kink in utility curve at the reference point, this specification is referred to as a local definition of loss aversion.

Local definitions of loss aversion provide a more straightforward measurement index for loss aversion and allow for risk preferences to be decomposed into the three elements of risk preference considered in this study: utility, decision weights and loss aversion. Global definitions of loss aversion, on the other hand, define loss aversion over the entire range of the utility function. They do not allow for the decomposition of risk preferences, can lead to ambiguous results and are generally considered less empirically useful. Readers are referred to Abdellaoui et al. (2007) for a comprehensive comparison of different measures of loss aversion. 


\subsubsection{Empirical findings}

Empirical findings on the median estimates of the model parameters are listed in Table 3.

Table 3 - Median Empirical Estimates of the Model Parameters

\begin{tabular}{|l|l|l|l|l|l|l|l|}
\hline Study & \multicolumn{1}{|c|}{$\theta^{+}$} & \multicolumn{1}{|c|}{$\theta^{-}$} & \multicolumn{1}{c|}{$\delta^{+}$} & \multicolumn{1}{c|}{$\gamma^{+}$} & $\delta^{-}$ & $\gamma^{-}$ & $\lambda$ \\
\hline Abdellaoui (2000) & .89 & .92 & .65 & .60 & .84 & .65 & \\
\hline Abdellaoui et al. (2005) & .91 & .96 & .98 & .83 & 1.35 & .84 & \\
\hline Abdellaoui et al. (2008) & .86 & 1.06 & & & & & 2.61 \\
\hline Andersen et al. (2006) & .81 & .80 & & & & & 1.07 \\
\hline Bleichrodt \& Pinto (2000) & & & & & & & \\
\hline Etchart-Vincent (2004) & & .97 & & & 1.10 & .84 & \\
\hline Etchart-Vincent (2009) & & & & & 1.36 & .75 & \\
\hline Fehr-Duda et al. (2006) & 1.01 & 1.05 & .87 & .51 & 1.07 & .53 & \\
\hline Gonzalez \& Wu (1999) & .49 & & .77 & .44 & & & \\
\hline Harrison \& Rutström (2009) & .71 & .72 & & & & & 1.38 \\
\hline Wu \& Gonzalez (1996) & & & .84 & .68 & & & \\
\hline Tversky \& Kahneman (1992) & .88 & .88 & & & & & 2.25 \\
\hline Tversky \& Fox (1995) & & & .77 & .69 & & & .98 \\
\hline Present Study & .95 & 1.00 & .98 & 1.38 & 1.77 & 1.34 \\
\hline
\end{tabular}




\section{CHAPTER 5 \\ METHODOLOGY}

This study adopts a repeated-measures design to measure the effect that visceral factors have on the way people interpret information in lottery-style decision tasks. This is determined by eliciting three distinct elements that define individual risk preference under CPT while participants experience varying degrees of visceral states. Seven separate OLS and fixed-effects regressions are then used to explore the effects of these factors on each of the model parameters.

There are three trials to this experiment. The first trial includes six sections: two eligibility questions, 16 demographic questions, a ten-question personality questionnaire, a four-question practice exercise, eight unipolar visual analogue scales and a 25-question risk elicitation questionnaire. The risk elicitation questionnaire includes 12 utility elicitation questions (6 gain and 6 loss), 10 decision weight elicitation questions (5 gain and 5 loss), and 3 mixed gamble questions to measure loss aversion. The second and third trials include only the visual analogue scales and the risk preference elicitation questions.

Prior to participating in the study, participants completed an initial intake survey to confirm eligibility criteria as well as to gather information on personality traits and demographic factors related to the parameters of interest. Exclusion criteria consists of anyone under the legal gambling age of 18 and anyone having suffered a brain injury as is it unclear how such injuries can influence the results.

Personality traits are characterized by the 'Big Five' and assessed using a 10-item measure (BFI-10) designed by Rammstedt \& John (2007). Sixteen demographic variables are also collected to account for individual characteristics as outlined in Appendix B.

The risk preference elicitation methods used measure all three elements independently using non-parametric methods as described by Wakker \& Deneffe (1996), Abdellaoui (2000) and Booij \& van de Kuilen (2009) aggregated into a single lottery-style questionnaire. Risk preference in gain, loss and mixed domains are elicited randomly over the course of a three-week period using an email notification prompting participants to answer a series of questions. In each trial, participants are asked to self-evaluate their current state with respect to the factors of 
interest before answering the lottery-style elicitation questions. Data from the visual analogue scales are used to attribute risk preferences to a specific state or combination of states.

To ensure comprehension and the consistency of responses, a 4-question comprehension test with forced responses and two instructional videos are embedded into the questionnaire. One video was made to explain how to answer the utility elicitation questions and another to explain the decision weight elicitation questions. In both videos, participants are shown an example and reminded that there is no right or wrong answer.

\subsection{Survey Design}

The survey was developed on the Qualtrics platform with some constraints limiting participants' ability to identify strictly dominated lotteries as an indifference point. All participants are presented with numeric and visual representations of gambles making explicit all relevant criteria. Figure 7 illustrates the utility elicitation questions presented to participants where $G, g$ and an initial value for $X_{i}$ are given. Participants are asked to identify a value, $X_{i-1}$, that would make them indifferent between the two gambles. Each series of questions are chained meaning that the participant's answer to the current question is used as a given value in the subsequent question. The structure of the risk preference elicitation questions is outlined in Table 5.

Figure 7 - Example of a Risk Elicitation Question

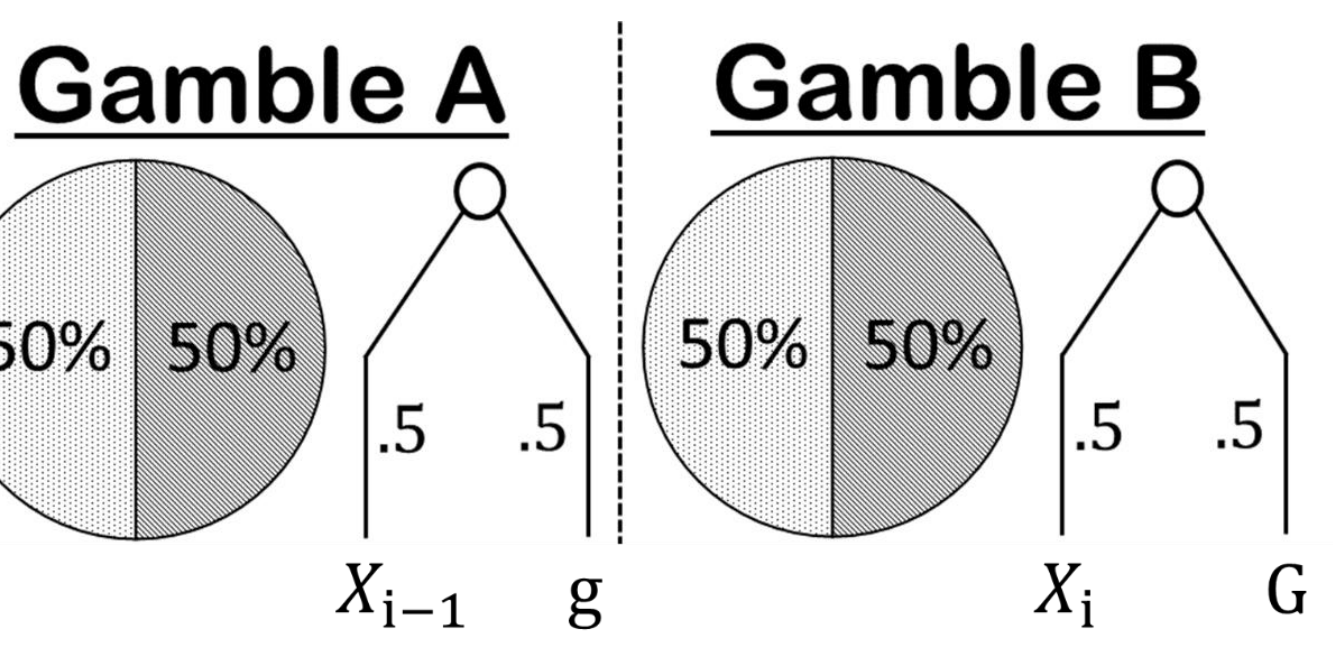

\subsection{Data Collection}

The purpose of this study is to understand how emotions occurring naturally through the course of daily activities affect decisions in the moment. While psychological primes administered in a 
laboratory setting were considered, I was concerned that participants would be subject to biases that would impair my ability to capture the true affect of these emotions. People have a systematic tendency to alter their behaviour when they know they're being observed which this study mitigates by collecting data in a more personal environment with little-to-no researcher influence.

Participants were recruited from the Ryerson Student Research Participant Pool through the SONA platform. Most of the participants are enrolled in an undergraduate business program at the Ted Rogers School of Management and received a maximum of two bonus percentage points applied towards a participating course for completing all three trials of the experiment. In total, 383 people participated in the first trial of the experiment, 164 participated in trials one and two, and 109 participated in all three trials.

Each participant was directed to answer a series of questions randomly over the course of a three-week period. Figure 14 in Appendix C illustrates the survey presented to participants. There were no time restrictions placed on the participants. On average, participants were able to complete trials one, two and three in 18.7 minutes, 12.6 minutes and 10.6 minutes, respectively, as listed in Table 4.

Table 4 - Average Time to Complete Each Trial of the Experiment

\begin{tabular}{|l|c|c|c|}
\hline & Trial 1 (min.) & Trial 2 (min.) & Trial 3 (min.) \\
\hline Median & 16.3 & 10.4 & 7.7 \\
\hline IQR & $11.3-21.7$ & $7.9-14.3$ & $5.2-11.8$ \\
\hline Mean & 18.7 & 12.6 & 10.6 \\
\hline S.D. & 13.2 & 7.9 & 8.7 \\
\hline
\end{tabular}

\subsection{Structure of Risk Preference Elicitation Questions}

Table 5 illustrates the structure and order of the risk elicitation questionnaire. The notation $\left(S_{1}, p, S_{2}\right) \sim\left(R_{1}, q, R_{2}\right)$ describes an indifference relationship where a participant is indifferent between prospect $\mathrm{S},\left(S_{1}, p, S_{2}\right)$, where outcomes $\mathrm{S}_{1}$ and $\mathrm{S}_{2}$ will occur with a probability, $p$ and $(1-p)$, respectively, and prospect $\mathrm{R},\left(R_{1}, q, R_{2}\right)$, where outcomes $\mathrm{R}_{1}$ and $\mathrm{R}_{2}$ will occur with a probability, $q$ and $(1-q)$, respectively. The variables in bold identify unknown variables to be determined by participants.

The risk preference elicitation questions are grouped into three categories: gain-gambles: $G, g, X_{0}, X_{i}>0$; loss-gambles: $L, l, Y_{0}, Y_{i}<0$; and mixed gambles with a possibility of either 
gain or loss: $X_{i}<0<Y_{i}$. The risk preference questionnaire can be further categorized into five sections: utility in the gain domain (questions 1-6), utility in the loss domain (questions 7-12), decision weights in the gain domain (questions 16-20), decision weights in the loss domain (questions 21-25), and loss aversion (questions 13-15).

\section{Table 5 - Structure of Risk Elicitation Questions}

\begin{tabular}{|c|c|c|c|c|c|c|c|}
\hline Question & $\begin{array}{l}\text { Prospect S } \\
\left(S_{1}, p, S_{2}\right)\end{array}$ & & $\begin{array}{l}\text { Prospect R } \\
\left(R_{1}, q, R_{2}\right)\end{array}$ & Question & $\begin{array}{l}\text { Prospect S } \\
\qquad\left(S_{2}\right)\end{array}$ & & $\begin{array}{l}\text { Prospect R } \\
\left(R_{1}, p, R_{2}\right)\end{array}$ \\
\hline 1 (Gains) & $\left(X_{1}, .5, g\right)$ & $\sim$ & $\left(X_{0}, .5, G\right)$ & 16 & $\left(X_{1}\right)$ & $\sim$ & $\left(X_{6}, \boldsymbol{p}_{1}, X_{0}\right)$ \\
\hline 2 (Gains) & $\left(X_{2}, .5, g\right)$ & $\sim$ & $\left(X_{1}, .5, G\right)$ & 17 & $\left(X_{2}\right)$ & $\sim$ & $\left(X_{6}, \boldsymbol{p}_{2}, X_{0}\right)$ \\
\hline 3 (Gains) & $\left(\boldsymbol{X}_{3}, .5, g\right)$ & $\sim$ & $\left(X_{2}, .5, G\right)$ & 18 & $\left(X_{3}\right)$ & $\sim$ & $\left(X_{6}, \boldsymbol{p}_{3}, X_{0}\right)$ \\
\hline 4 (Gains) & $\left(\boldsymbol{X}_{4}, .5, g\right)$ & $\sim$ & $\left(X_{3}, .5, G\right)$ & 19 & $\left(X_{4}\right)$ & $\sim$ & $\left(X_{6}, \boldsymbol{p}_{4}, X_{0}\right)$ \\
\hline 5 (Gains) & $\left(\boldsymbol{X}_{5}, .5, g\right)$ & $\sim$ & $\left(X_{4}, .5, G\right)$ & 20 & $\left(X_{5}\right)$ & $\sim$ & $\left(X_{6}, \boldsymbol{p}_{5}, X_{0}\right)$ \\
\hline 6 (Gains) & $\left(\boldsymbol{X}_{6}, .5, g\right)$ & $\sim$ & $\left(X_{5}, .5, G\right)$ & 21 & $\left(Y_{1}\right)$ & $\sim$ & $\left(Y_{6}, \boldsymbol{q}_{\mathbf{1}}, Y_{0}\right)$ \\
\hline 7 (Losses) & $\left(\boldsymbol{Y}_{1}, .5, l\right)$ & $\sim$ & $\left(Y_{0}, .5, L\right)$ & 22 & $\left(Y_{2}\right)$ & $\sim$ & $\left(Y_{6}, \boldsymbol{q}_{2}, Y_{0}\right)$ \\
\hline 8 (Losses) & $\left(Y_{2}, .5, l\right)$ & $\sim$ & $\left(Y_{1}, .5, L\right)$ & 23 & $\left(Y_{3}\right)$ & $\sim$ & $\left(Y_{6}, \boldsymbol{q}_{3}, Y_{0}\right)$ \\
\hline 9 (Losses) & $\left(\boldsymbol{Y}_{3}, .5, l\right)$ & $\sim$ & $\left(Y_{2}, .5, L\right)$ & 24 & $\left(Y_{4}\right)$ & $\sim$ & $\left(Y_{6}, \boldsymbol{q}_{4}, Y_{0}\right)$ \\
\hline 10 (Losses) & $\left(\boldsymbol{Y}_{4}, .5, l\right)$ & $\sim$ & $\left(Y_{3}, .5, L\right)$ & 25 & $\left(Y_{5}\right)$ & $\sim$ & $\left(Y_{6}, \boldsymbol{q}_{\mathbf{5}}, Y_{0}\right)$ \\
\hline 11 (Losses) & $\left(Y_{5}, .5, l\right)$ & $\sim$ & $\left(Y_{4}, .5, L\right)$ & & & & \\
\hline 12 (Losses) & $\left(\boldsymbol{Y}_{6}, .5, l\right)$ & $\sim$ & $\left(Y_{5}, .5, L\right)$ & & & & \\
\hline 13 (Gains) & $(b, .5,0)$ & $\sim$ & $\left(X_{1}, .5, X_{0}\right)$ & & & & \\
\hline 14 (Losses) & $(c, .5,0)$ & $\sim$ & $\left(Y_{0}, .5, Y_{1}\right)$ & & & & \\
\hline 15 (Mixed) & $\left(\boldsymbol{d}, .5, Y_{1}\right)$ & $\sim$ & $\left(X_{0}, .5, Y_{0}\right)$ & & & & \\
\hline
\end{tabular}

Prospect $\mathrm{S}=$ Safe choice

Prospect $\mathrm{R}=$ Risky choice

\subsection{Basic Utility Elicitation Method}

Assuming a representing function for preference relations exists (see von Neumann \&

Morgenstern, 1944), an indifference relation translates to an equality of the underlying functional representation $[X \sim Y \Leftrightarrow u(X)=u(Y)]$ and an individual's utility function can be derived. The uniqueness of utility functions up to positive linear transformations makes possible a simple method to derive a utility function from preference relations. By normalizing the gamble outcomes so that $X_{1}=1$ and $X_{0}=0$ and setting $u(1)=1$ and $u(0)=0$, the utility value of the certainty equivalent can be calculated under EU.

$$
\begin{aligned}
& \alpha X_{1}+(1-\alpha) X_{0} \sim Z_{i} \Leftrightarrow \alpha u\left(X_{1}\right)+(1-\alpha) u\left(X_{0}\right)=u\left(Z_{i}\right) \\
& \Rightarrow \alpha u(1)+(1-\alpha) u(0)=u\left(Z_{i}\right) \\
& \Rightarrow \alpha=u\left(Z_{i}\right)
\end{aligned}
$$


Under EU the individual's utility for the certain amount is equal to the probability that makes the decision maker indifferent between the normalized outcomes in a two-prospect lottery and the certainty equivalent. This process can then be repeated using a chaining method until a sufficiently complete utility function has been constructed (von Neumann \& Morgenstern, 1944; Torgerson, 1959; Keeney \& Raiffa, 1976). To normalize the outcomes and solve for the indifference points we must assume that utility is unique up to positive linear transformations which implies the cardinality of utility. Additionally, we assume that lotteries are nondegenerate, meaning that $X_{1} \neq X_{0}$ or that $\alpha \neq 1$ or 0 , to ensure that there is no certainty about a future state. This is known as a paired-gamble method and avoids biases associated with the certainty effect by replacing certainty equivalents with other gambles (Officer \& Halter, 1968; Kahneman \& Tversky, 1979; Schmidt, 1998).

$$
\left[X_{1}, \alpha, X_{0}\right] \prec, \sim,>\left[X_{i}, \beta, X_{j}\right] \Leftrightarrow \alpha X_{1}+(1-\alpha) X_{0}<,=,>\beta X_{i}+(1-\beta) X_{j}
$$

While the methods described are simple and relatively easy to compute, many have found the risk preferences they elicit to be unreliable. A significant drawback of most of the earlier elicitation methods is that they treat probabilities as linear which introduces a distortionary effect on utility functions and systematic biases the utility measurements (Karmarkar, 1978; McCord \& de Neufville, 1986; Holt, 1986; Abdellaoui et al., 2008).

In response to these effects and calls for non-EU utility elicitation methods, Wakker \& Deneffe (1996) developed the gamble-tradeoff (TO) method. This is a non-parametric elicitation method that eliminates probability distortions on the elicited utility function. It does so by eliciting indifference values in a series of paired-gambles that are designed with a standard outcome sequence that equally spaces elicited outcomes in utility units. The standardizing of utility values in this way enables a simple derivation of the utility function to the desired level of accuracy with $u\left(X_{i}\right)=i / n$.

The major advantage to this method is that the decision weights cancel out of the equation. This holds probability constant which eliminates probability distortion in the utility function and enables researchers to measure the utility of lotteries with known and unknown probabilities. The following three equations illustrate this effect: 


$$
\left[X_{i-1}, \alpha, G\right] \sim\left[X_{i}, \alpha, g\right] \Leftrightarrow w(\alpha) u\left(X_{i-1}\right)+[1-w(\alpha)] u(G)=w(\alpha) u\left(X_{i}\right)+[1-w(\alpha)] u(g)
$$

$$
[1-w(\alpha)][u(G)-u(g)]=w(\alpha)\left[u\left(X_{i}\right)-u\left(X_{i-1}\right)\right]
$$

$\left[X_{j-1}, \alpha, G\right] \sim\left[X_{j}, \alpha, g\right] \Leftrightarrow w(\alpha) u\left(X_{j-1}\right)+[1-w(\alpha)] u(G)=w(\alpha) u\left(X_{j}\right)+[1-w(\alpha)] u(g)$

$$
[1-w(\alpha)][u(G)-u(g)]=w(\alpha)\left[u\left(X_{j}\right)-u\left(X_{j-1}\right)\right]
$$

$w(\alpha)\left[u\left(X_{j}\right)-u\left(X_{j-1}\right)\right]=w(\alpha)\left[u\left(X_{i}\right)-u\left(X_{i-1}\right)\right] \Rightarrow u\left(X_{j}\right)-u\left(X_{j-1}\right)=u\left(X_{i}\right)-u\left(X_{i-1}\right)$

where $0 \leq g<G<X_{i-1}<X_{i} \leq X_{j-1}<X_{j}$ such that $g, G, X_{i} \in X$ and $\alpha \in(0,1)$. When combining these equations, the difference in utility between $\left(X_{i} ; X_{i-1}\right)$ and $\left(X_{j} ; X_{j-1}\right)$ are the same for all $X_{i}, X_{j}$ in the outcome sequence $\left(X_{0}, \ldots, X_{i}, \ldots, X_{j}, \ldots, X_{n}\right)$. Furthermore, if $X_{j-1}=X_{i}$ then $X_{j}=X_{i+1}$ and $X_{i}$ becomes the midpoint outcome between $X_{i-1}$ and $X_{i+1}$ utility values so that $u\left(X_{i+1}\right)-u\left(X_{i}\right)=u\left(X_{i}\right)-u\left(X_{i-1}\right)$.

Non-parametric elicitation methods such as this are desirable because they elicit measurements that are unaffected by imposed parametric assumptions and because they provide a direct link between observed choices and utilities (Abdellaoui et al., 2007; 2008). Since its release in 1996 the TO method has become the gold standard in utility elicitation and believed to be significantly less susceptible to bias than parametric methods (Wakker \& Deneffe, 1996; Wakker, 2004a). The TO method was selected for the current study because it eliminates probability distortions and can measure individual utilities in the domain of either gains or losses under CPT.

Utility parameters in the current study are elicited using a series of 50/50 gambles. This is done under the assumption that probability distortions around .5 are minimal to reduce probability distortions when eliciting utility functions and ensure that elicited preferences are confined to the realm of utilities (Binswanger, 1981; Eckel \& Grossman, 2002; Quiggin, 1981).

\subsection{Decision Weight Elicitation Method}

The current study adopts a non-parametric decision weight elicitation method developed by Abdellaoui (2000) that effectively avoids biases resulting from the certainty effect and other distortionary effects explained in the previous section. This method leverages the utility values associated with the outcomes identified as indifference points using the TO method explained in 
the previous section. This information is then used to assign the series of probability values elicited in decision weight elicitation questions across a standard sequence of decision weights. From this, it is then possible to estimate the shape of each participant's probability weighting function in both the gain and loss domains.

Indifference relations under this method are identified using a probability equivalence method where participants identify a probability, $\mathrm{p}_{i} \in(0,1)$, that makes them indifferent between receiving an outcome, $X_{i}$, with certainty and the gamble $\left[X_{n}, \mathrm{p}_{i}, X_{0}\right]$. Under CPT, this indifference relation implies that the decision weight of the elicited probability value is equal to the ratio of the position of $u\left(X_{i}\right)$ in the standard sequence of utility units, $i$, to the total number of positions in the standard sequence, $n$.

$$
\begin{gathered}
{\left[X_{n}, \mathrm{p}_{i}, X_{0}\right] \sim\left[X_{i}, 1, X_{0}\right]} \\
w\left(\mathrm{p}_{i}\right)=\frac{u\left(X_{i}\right)-u\left(X_{0}\right)}{u\left(X_{n}\right)-u\left(X_{0}\right)}=\frac{i}{n}
\end{gathered}
$$

where $u\left(X_{0}\right)$ and $u\left(X_{n}\right)$ are the smallest and largest utility values, respectfully, in the standard sequence of utility units and $u\left(X_{i}\right)$ is the utility value corresponding to the $i^{\text {th }}$ elicited outcome obtained using the TO method. For example, if there are six indifference points elicited under the TO method, the probability that makes the participant indifferent between $\left[X_{6}, \mathrm{p}_{i}, X_{0}\right]$ and $\left[X_{3}, 1, X_{0}\right]$ would be equal to a decision weight of $3 / 6$.

Once all indifference points are observed over the entire range of elicited outcomes, $X_{1}, \ldots, X_{n-1}$, each elicited probability is matched to its corresponding decision weight and inferences can be made regarding the shape of participants' probability weighting function.

\subsection{Loss Aversion Elicitation Method}

Several studies further extended the non-parametric elicitation methods to measure one of the most pervasive effects in decision making, loss aversion (Abdellaoui, Bleichrodt \& Paraschiv, 2007; Booij, 2009; Booij \& van de Kuilen, 2009). Since loss aversion describes how decision makers perceive losses relative to gains, it is necessary to use an elicitation method capable of measuring preferences over losses and gains simultaneously. While the TO method can measure 
individual utilities in either the gain or loss domain, it does not allow for utilities to be measured across both simultaneously (Abdellaoui et al. 2007).

Three mixed gambles are included in the risk preference elicitation questionnaire to scale the previously elicited utility values in the gain domain to those in the loss domain and derive an index for loss aversion: questions 13, 14 and 15 in the 'Structure of Elicitation Questions' section. The values for $w^{+}(.5), w^{-}(.5), u(b), u(c), u\left(X_{1}\right), u\left(Y_{1}\right)$ and $u(d)$ in the following equations are either drawn from the previously elicited values or approximated using linear interpolation.

This study adopts a three-stage procedure developed by Booij (2009) to calculate the loss aversion parameter. The first stage uses question 13 to determine the utility of $X_{0}$ in terms of the standard sequence of utility units in the gain domain. I ask participants to identify an amount, $b>X_{1}$, that makes them indifferent between the following two prospects: $[b, .5,0] \sim\left[X_{1}, .5, X_{0}\right]$. Under CPT, this indifference implies:

$$
u\left(X_{0}\right)=\frac{w^{+}(.5)}{1-w^{+}(.5)}\left[u(b)-u\left(X_{1}\right)\right]
$$

The second stage uses the same procedure in the loss domain to determine the utility of $Y_{0}$ in terms of the standard sequence of utility units in the loss domain. Question 14 asks participants to identify an amount, $c<Y_{1}$, that makes them indifferent between the following two prospects: $[0, .5, c] \sim\left[Y_{0}, .5, Y_{1}\right]$. Again, this implies:

$$
u\left(Y_{0}\right)=\frac{w^{-}(.5)}{1-w^{-}(.5)}\left[u(c)-u\left(Y_{1}\right)\right]
$$

The first two stages of this procedure connect the utility curves in the gain and loss domains through zero making it observable over $\left\{\left[Y_{6}, u\left(Y_{6}\right)\right], \ldots,\left[Y_{0}, u\left(Y_{0}\right)\right], 0,\left[X_{0}, u\left(X_{0}\right)\right], \ldots,\left[X_{6}, u\left(X_{6}\right)\right]\right\}$. Because utilities in the two domains were elicited separately and without making parametric assumptions, the standard sequence of utility units in the gain domain may be a different scale than that in the loss domain.

The third stage in this process acts to reconcile the utility scales by measuring utilities across gains and losses simultaneously. This is achieved with the use of a mixed gamble, question 15 in 
the 'Structure of Elicitation Questions' section. In this stage, participants are asked to identify an amount, $d>X_{0}$, that makes them indifferent between the following two prospects: $\left[d, .5, Y_{1}\right] \sim$ $\left[X_{0}, .5, Y_{0}\right]$. This implies:

$$
u\left(Y_{0}\right)-u\left(Y_{1}\right)=\frac{w^{+}(.5)}{w^{-}(.5)}\left[u(d)-u\left(X_{0}\right)\right]
$$

This equation is interpreted as one standard sequence of utility units in the loss domain is equal to some multiple of one standard sequence of utility units in the gain domain. Figure 8 shows an example of an unscaled utility function where one utility unit in the loss domain is equal to approximately $7 / 3$ utility units in the gain domain.

Figure 8 - Unscaled Utility Function

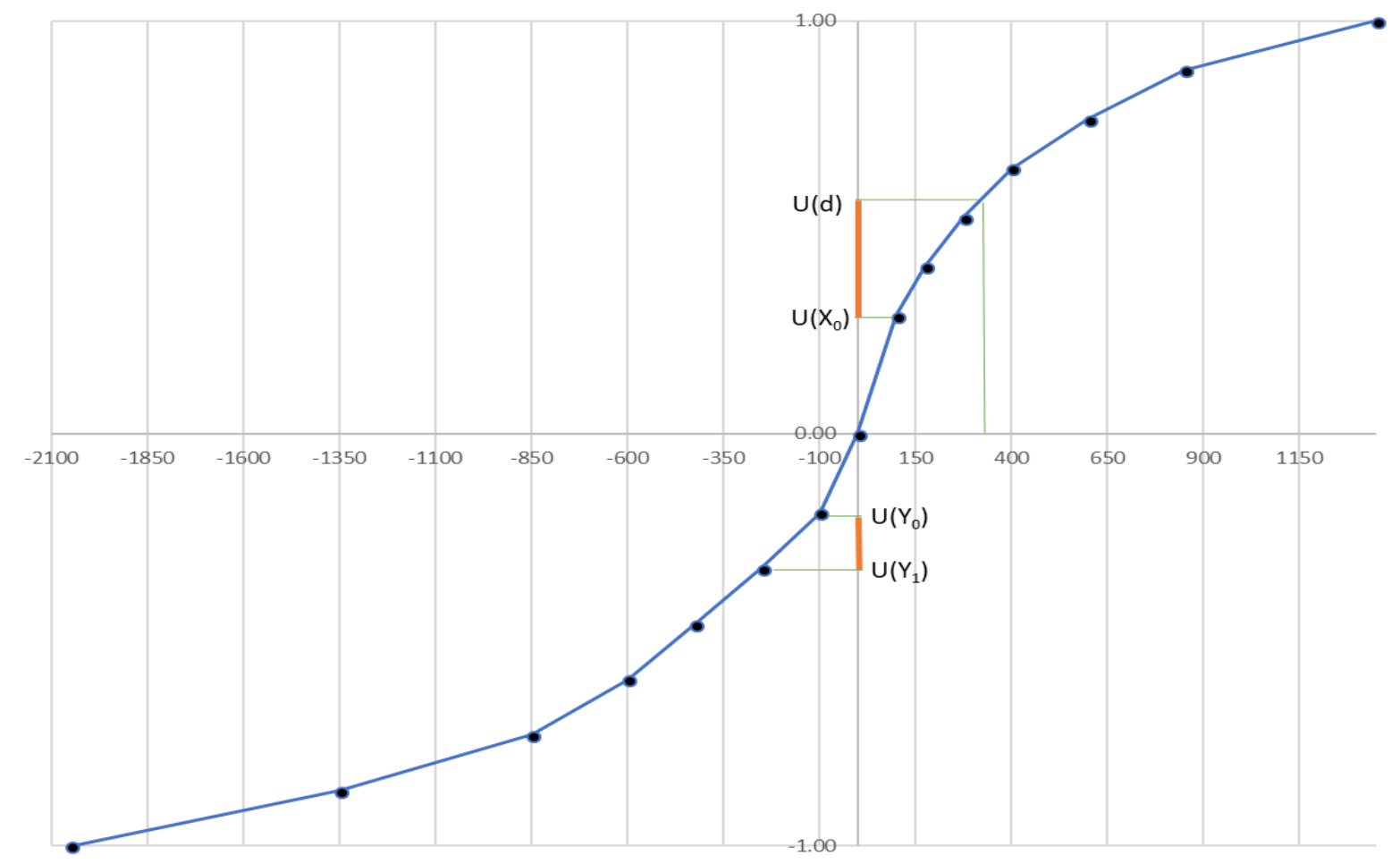

Once the utility units are scaled the loss aversion index can be determined by inserting the scaled utility values corresponding to outcomes $X_{0}$ and $Y_{0}$, into the following equation:

$$
\lambda=\frac{u\left(Y_{0}\right) X_{0}}{u\left(X_{0}\right) Y_{0}}
$$


where $u\left(Y_{0}\right)$ and $u\left(X_{0}\right)$ are the scaled utility values associated with outcomes $Y_{0}$ and $X_{0}$, respectively.

\subsection{Measuring Visceral Factors}

This study takes a distinct-states approach that treats emotions and other visceral factors as discrete events while allowing for factor interaction. The intensity level of the visceral factors under investigation are measured using a self-assessment method derived from the literature. Eight separate unipolar visual analogue scales are presented to participants as depicted in Figure 9 below and Figure 14 in Appendix C. Each participant in each trial of the experiment is asked to rate their current arousal level along each of the dimensions by sliding the bar from left to right. The default value for each of the visceral factors is zero which corresponds to an intensity level of "Not at all".

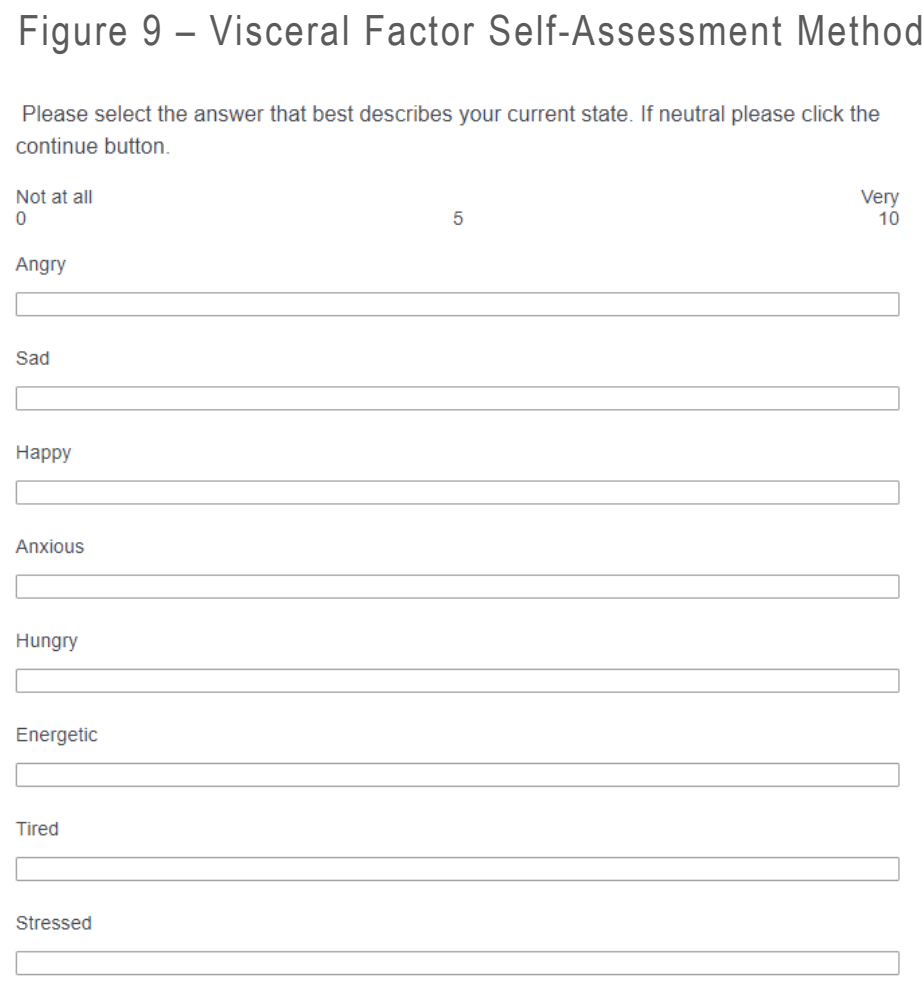

Information is collected on the type and intensity of eight visceral factors: anger, sadness, happiness, anxiety, hunger, energy, tiredness and stress. Anxiety is measured using a unipolar visual analogue scale anchored with the terms "Not at all anxious" on the left and "Very anxious" on the right. This method has been shown to be an adequate replacement for the Spielberger State Trait Anxiety Inventory commonly used to assess anxiety (Daveya et al. 2007). 
Anger, sadness, happiness, hunger, energy, tiredness and stress are all measured similarly with the terms "Not at all" on the left and "Very" on the right. The visceral factor measurements were recorded in one-unit increments with "Not at all" corresponding to an arousal level of 0/10 and "Very" corresponding to an arousal level of 10/10.

The following table lists the summary statistics for visceral factor measurements and an additional row labelled 'Exp. V.F.' to show the percent of the sample that identified as experiencing some level of arousal in each of the visceral factors. The dataset used to produce the summary statistics below is also used for the non-linear PCA and consists of 269 subjects from the first trial of the experiment. The minimum and maximum levels of arousal for all visceral factors are 0 and 10, respectively, indicating that a full spectrum of arousal levels across all visceral factors are represented in the sample.

Table 6 - Visceral Factor Summary Statistics

\begin{tabular}{|l|c|c|c|c|c|c|c|c|}
\hline & Anger & Sadness & Anxiety & Happiness & Tiredness & Energy & Hunger & Stress \\
\hline Exp. V.F. (\%) & 37.37 & 47.06 & 65.05 & 79.24 & 83.39 & 67.82 & 66.09 & 78.20 \\
\hline Mean & 1.32 & 1.79 & 3.09 & 4.49 & 4.95 & 2.95 & 3.62 & 4.43 \\
\hline Std. Dev. & 2.167 & 2.498 & 2.946 & 3.171 & 3.365 & 2.831 & 3.422 & 3.377 \\
\hline Median & 0 & 0 & 3 & 5 & 5 & 3 & 3 & 5 \\
\hline IQR & $0-2$ & $0-3$ & $0-5$ & $1-7$ & $2-8$ & $0-5$ & $0-7$ & $1-7$ \\
\hline
\end{tabular}

\subsection{Personality Traits}

In addition to demographic and visceral factors I also measured five broad dimensions of personality: openness to experience, conscientiousness, extraversion, agreeableness, and neuroticism. The big five personality traits are one of the oldest and most popular models of personality and considered to reflect stable patterns of behaviour. Recent studies have shown that personality traits directly affect individual risk preference and are also linked to both stress and emotion (Cahlíková \& Cingl, 2017; Van Der Zee et al. 2002). Controlling for personality traits is a common practice in similar experiments. Some researchers recommend that all studies examining the effect of visceral factors on behaviour address relevant personality traits (Campbell \& Ehlert, 2012).

The more common tools available to measure the big five personality traits include Costa and McCrae's (1992) 240-item NEO Personality Inventory, Revised (NEO-PI-R), a 50-item measure from the International Personality Item Pool (Costa and McCrae, 1992; Goldberg et al. 2006) and the 44-item measure (BFI-44) developed by Benet-Martinez \& John (1998). 
Given the erratic and transitory nature of emotions, a shorter 10-item measure (BFI-10) was used in this study to measure the big five personality traits (Rammstedt \& John, 2007). This tool uses 5-point Likert scales to measure the five personality traits along a scale from one to five. Since each personality trait is determined by two questions, traits are measured in .5-unit increments.

According to a comparison study, the BFI-10 scores at least as high in all relevant criteria as the Ten Item Personality Inventory (TIPI) developed by Gosling et al. (2003) (Rammstedt, \& John, 2007). For comparisons between the BFI-10 and other more comprehensive assessments, readers are referred to Rammstedt \& John (2007) and Gosling et al. (2003). Table 7 lists the summary statistics for each of the big five personality traits in the sample of 269 participants.

Table 7 - Personality Trait Summary Statistics

\begin{tabular}{|l|c|c|c|c|c|}
\hline & Openness & Conscientiousness & Extraversion & Agreeableness & Neuroticism \\
\hline Mean & 3.054 & 3.364 & 3.199 & 3.496 & 3.182 \\
\hline Std. Dev. & .6492 & .7526 & .9185 & .7999 & .9286 \\
\hline Median & 3 & 3.5 & 3 & 3.5 & 3 \\
\hline IQR & $2.5-3.5$ & $3-4$ & $2.5-4$ & $3-4$ & $2.5-4$ \\
\hline Min. & 1 & 1.5 & 1 & 1.5 & 1 \\
\hline Max & 4.5 & 5 & 5 & 5 & 5 \\
\hline
\end{tabular}




\section{CHAPTER 6}

\section{ANALYSIS AND RESULTS}

\subsection{Demographic Variable Summary Statistics}

Table 8 presents the summary statistics for the numeric demographic variables in the sample of 269 participants. On average, participants are 20 years of age earning $\$ 8,000$ annually and living in households with three other individuals. This is consistent with what I would expect in a sample of mostly undergraduate business students in Toronto, Ont. Table 9 presents the percentage of each of the categorical demographic variables in the sample along with the mean self-assessed visceral factor arousal level. Roughly two-thirds of the sample are female, have no investing experience and/or identify as having some college or university education. 14\% of participants have dependents and only a small percentage of participants identify as the head of their household. Close to $94 \%$ of participants either work part-time or identify as full-time students and roughly one-third of participants are working professionals. Lastly, there were no retired participants or post-graduates in the sample.

\section{Table 8 - Numeric Demographic Variable Summary Statistics}

\begin{tabular}{|l|l|l|l|l|l|}
\hline & Age (years) & Yearly Income (\$) & Gross Wealth (\$) & Net Wealth (\$) & \# Ppl in House \\
\hline Mean & 20.27 & 8,273 & 7,404 & 5,897 & 4.01 \\
\hline Std. Dev. & 2.062 & 10,349 & 10,906 & 8,835 & 1.379 \\
\hline Median & 20 & 6,000 & 1,000 & 750 & 4 \\
\hline IQR & $19-21$ & $0-11,000$ & $0-10,300$ & $0-10,000$ & $3-5$ \\
\hline Min. & 18 & 0 & 0 & $-20,000$ & 1 \\
\hline Max & 30 & 60,000 & 60,000 & 50,000 & 10 \\
\hline
\end{tabular}

Table 9 - Categorical Demographic Variable Summary Statistics

\begin{tabular}{|l|c|c|c|c|c|c|c|c|c|}
\hline & \multirow{2}{*}{$\%$} & \multicolumn{7}{|c|}{ Mean Self-Assessed Arousal Level (Std. Dev.) } \\
\cline { 3 - 9 } & & Anger & Sadness & Anxiety & Happiness & Tiredness & Energy & Hunger & Stress \\
\hline Female & 63.9 & 1.20 & 1.83 & 3.25 & 4.23 & 5.28 & 2.69 & 3.42 & 4.58 \\
& & $(2.175)$ & $(2.567)$ & $(3.072)$ & $(3.236)$ & $(3.374)$ & $(2.844)$ & $(3.439)$ & $(3.464)$ \\
\hline Male & 36.1 & 1.55 & 1.73 & 2.81 & 4.97 & 4.36 & 3.40 & 3.98 & 4.18 \\
& & $(2.146)$ & $(2.383)$ & $(2.702)$ & $(3.012)$ & $(3.282)$ & $(2.764)$ & $(3.382)$ & $(3.218)$ \\
\hline Investing & 30.1 & 1.02 & 1.51 & 2.94 & 4.90 & 4.81 & 3.44 & 3.79 & 4.19 \\
Experience & & $(1.877)$ & $(2.308)$ & $(2.934)$ & $(3.415)$ & $(3.465)$ & $(2.846)$ & $(3.527)$ & $(3.321)$ \\
\hline No Investing & 69.9 & 1.45 & 1.91 & 3.16 & 4.32 & 5.01 & 2.73 & 3.55 & 4.54 \\
Experience & & $(2.274)$ & $(2.572)$ & $(2.957)$ & $(3.053)$ & $(3.329)$ & $(2.804)$ & $(3.383)$ & $(3.404)$ \\
\hline Has Dependents & 13.8 & 1.43 & 1.57 & 2.81 & 5.73 & 4.76 & 4.35 & 4.38 & 4.46 \\
& & $(2.267)$ & $(2.193)$ & $(2.767)$ & $(3.006)$ & $(3.303)$ & $(2.889)$ & $(3.722)$ & $(3.322)$ \\
\hline No Dependents & 86.2 & 1.31 & 1.83 & 3.14 & 4.30 & 4.98 & 2.72 & 3.50 & 4.43 \\
& & $(2.156)$ & $(2.546)$ & $(2.977)$ & $(3.158)$ & $(3.381)$ & $(2.762)$ & $(3.365)$ & $(3.393)$ \\
\hline Head of & 2.6 & 1.00 & .71 & 2.57 & 3.00 & 2.57 & 2.29 & 3.57 & 3.71 \\
Household & & $(1.915)$ & $(.951)$ & $(2.440)$ & $(3.317)$ & $(3.952)$ & $(3.094)$ & $(3.505)$ & $(4.424)$ \\
\hline
\end{tabular}




\begin{tabular}{|c|c|c|c|c|c|c|c|c|c|}
\hline $\begin{array}{l}\text { Not Head of } \\
\text { House. }\end{array}$ & 97.4 & $\begin{array}{c}1.33 \\
(2.176)\end{array}$ & $\begin{array}{c}1.82 \\
(2.521)\end{array}$ & $\begin{array}{c}3.11 \\
(2.961)\end{array}$ & $\begin{array}{c}4.53 \\
(3.164)\end{array}$ & $\begin{array}{c}5.02 \\
(3.333)\end{array}$ & $\begin{array}{c}2.97 \\
(2.828)\end{array}$ & $\begin{array}{c}3.63 \\
(3.427)\end{array}$ & $\begin{array}{c}4.45 \\
(3.353)\end{array}$ \\
\hline \multicolumn{10}{|l|}{ Education } \\
\hline High School & 25.7 & $\begin{array}{c}1.51 \\
(2.220)\end{array}$ & $\begin{array}{c}1.55 \\
(2.336)\end{array}$ & $\begin{array}{c}3.06 \\
(2.833)\end{array}$ & $\begin{array}{c}5.61 \\
(2.937)\end{array}$ & $\begin{array}{c}4.97 \\
(3.258)\end{array}$ & $\begin{array}{c}3.67 \\
(2.774)\end{array}$ & $\begin{array}{c}4.01 \\
(3.470)\end{array}$ & $\begin{array}{c}4.57 \\
(3.367)\end{array}$ \\
\hline $\begin{array}{l}\text { Some College or } \\
\text { University }\end{array}$ & 68.4 & $\begin{array}{c}1.28 \\
(2.164)\end{array}$ & $\begin{array}{c}1.89 \\
(2.590)\end{array}$ & $\begin{array}{c}3.08 \\
(3.024)\end{array}$ & $\begin{array}{c}4.14 \\
(3.116)\end{array}$ & $\begin{array}{c}4.97 \\
(3.412)\end{array}$ & $\begin{array}{c}2.69 \\
(2.793)\end{array}$ & $\begin{array}{c}3.54 \\
(3.397)\end{array}$ & $\begin{array}{c}4.35 \\
(3.388)\end{array}$ \\
\hline $\begin{array}{l}\text { Post-Secondary } \\
\text { Degree or } \\
\text { Certificate }\end{array}$ & 5.2 & $\begin{array}{c}.86 \\
(1.875)\end{array}$ & $\begin{array}{c}1.43 \\
(1.651)\end{array}$ & $\begin{array}{c}3.43 \\
(2.593)\end{array}$ & $\begin{array}{c}3.50 \\
(3.956)\end{array}$ & $\begin{array}{c}4.79 \\
(3.423)\end{array}$ & $\begin{array}{c}2.29 \\
(2.946)\end{array}$ & $\begin{array}{c}2.64 \\
(3.608)\end{array}$ & $\begin{array}{c}4.93 \\
(3.518)\end{array}$ \\
\hline \multicolumn{10}{|l|}{$\begin{array}{l}\text { Employment } \\
\text { Status }\end{array}$} \\
\hline Not Employed & 2.6 & $\begin{array}{c}1.00 \\
(1.732)\end{array}$ & $\begin{array}{c}.71 \\
(1.890)\end{array}$ & $\begin{array}{c}2.14 \\
(2.410)\end{array}$ & $\begin{array}{c}5.71 \\
(4.192)\end{array}$ & $\begin{array}{c}4.86 \\
(3.132)\end{array}$ & $\begin{array}{c}4.14 \\
(3.237)\end{array}$ & $\begin{array}{c}3.14 \\
(4.018)\end{array}$ & $\begin{array}{c}3.29 \\
(2.928)\end{array}$ \\
\hline Student & 45.4 & $\begin{array}{c}1.36 \\
(2.167)\end{array}$ & $\begin{array}{c}1.62 \\
(2.261)\end{array}$ & $\begin{array}{c}2.85 \\
(2.857) \\
\end{array}$ & $\begin{array}{c}4.37 \\
(3.163)\end{array}$ & $\begin{array}{c}4.56 \\
(3.411)\end{array}$ & $\begin{array}{c}3.07 \\
(2.931)\end{array}$ & $\begin{array}{c}3.69 \\
(3.621)\end{array}$ & $\begin{array}{c}3.90 \\
(3.482)\end{array}$ \\
\hline Part-Time & 48.3 & $\begin{array}{c}1.29 \\
(2.165) \\
\end{array}$ & $\begin{array}{c}2.02 \\
(2.756) \\
\end{array}$ & $\begin{array}{c}3.38 \\
(3.053) \\
\end{array}$ & $\begin{array}{c}4.54 \\
(3.053)\end{array}$ & $\begin{array}{c}5.17 \\
(3.316) \\
\end{array}$ & $\begin{array}{c}2.77 \\
(2.655) \\
\end{array}$ & $\begin{array}{c}3.59 \\
(3.184) \\
\end{array}$ & $\begin{array}{c}4.80 \\
(3.178) \\
\end{array}$ \\
\hline Full-Time & 3.7 & $\begin{array}{c}1.50 \\
(2.718)\end{array}$ & $\begin{array}{c}1.70 \\
(1.947)\end{array}$ & $\begin{array}{c}3.00 \\
(3.232)\end{array}$ & $\begin{array}{c}4.60 \\
(4.274)\end{array}$ & $\begin{array}{c}7.00 \\
(3.018)\end{array}$ & $\begin{array}{c}3.00 \\
(3.651)\end{array}$ & $\begin{array}{c}3.60 \\
(4.033)\end{array}$ & $\begin{array}{c}7.00 \\
(3.464)\end{array}$ \\
\hline \multicolumn{10}{|l|}{ Occupation } \\
\hline Self-Employed & 4.1 & $\begin{array}{c}1.27 \\
(2.453)\end{array}$ & $\begin{array}{c}1.73 \\
(2.328)\end{array}$ & $\begin{array}{c}3.18 \\
(3.060)\end{array}$ & $\begin{array}{c}5.64 \\
(2.730) \\
\end{array}$ & $\begin{array}{c}4.18 \\
(3.401)\end{array}$ & $\begin{array}{c}4.73 \\
(3.069)\end{array}$ & $\begin{array}{c}4.36 \\
(3.443)\end{array}$ & $\begin{array}{c}5.45 \\
(3.560)\end{array}$ \\
\hline $\begin{array}{l}\text { Working } \\
\text { Professional }\end{array}$ & 31.2 & $\begin{array}{c}1.36 \\
(2.274)\end{array}$ & $\begin{array}{c}1.81 \\
(2.614)\end{array}$ & $\begin{array}{c}3.42 \\
(3.007) \\
\end{array}$ & $\begin{array}{c}5.25 \\
(3.127) \\
\end{array}$ & $\begin{array}{c}5.37 \\
(3.449) \\
\end{array}$ & $\begin{array}{c}3.33 \\
(2.778) \\
\end{array}$ & $\begin{array}{c}4.01 \\
(3.562)\end{array}$ & $\begin{array}{c}4.81 \\
(3.493) \\
\end{array}$ \\
\hline Other & 64.3 & $\begin{array}{c}1.32 \\
(2.112) \\
\end{array}$ & $\begin{array}{c}1.80 \\
(2.468) \\
\end{array}$ & $\begin{array}{c}2.94 \\
(2.922) \\
\end{array}$ & $\begin{array}{c}4.08 \\
(3.141) \\
\end{array}$ & $\begin{array}{c}4.80 \\
(3.328) \\
\end{array}$ & $\begin{array}{c}2.66 \\
(2.794) \\
\end{array}$ & $\begin{array}{c}3.41 \\
(3.348) \\
\end{array}$ & $\begin{array}{c}4.21 \\
(3.293) \\
\end{array}$ \\
\hline Total & 100.0 & $\begin{array}{c}1.32 \\
(2.167) \\
\end{array}$ & $\begin{array}{c}1.79 \\
(2.498) \\
\end{array}$ & $\begin{array}{c}3.09 \\
(2.946) \\
\end{array}$ & $\begin{array}{c}4.49 \\
(3.171) \\
\end{array}$ & $\begin{array}{c}4.95 \\
(3.365) \\
\end{array}$ & $\begin{array}{c}2.95 \\
(2.831) \\
\end{array}$ & $\begin{array}{c}3.62 \\
(3.422) \\
\end{array}$ & $\begin{array}{c}4.43 \\
(3.377)\end{array}$ \\
\hline
\end{tabular}

\subsection{Utility Analysis}

\subsubsection{Non-Parametric Utility Analysis}

The curvature of each participant's utility function is first analyzed without making any assumptions about parametric form. This analysis is used to assess the overall utility curvatures in the sample and provide a point of comparison to assess the accuracy of the parametric specification used in this study. To be included in the utility analysis participants must provide logical responses to at least four out of the six elicitation questions in either the utility gain or utility loss sections.

This analysis began by calculating seven differences between elicited outcomes and performing a pooled samples t-test to determine if each successive difference in elicited outcomes was significantly different from the last. While constraints built into the questionnaire ensure that the absolute magnitude of each elicited outcome is equal to or greater than the last, this difference is only considered significant if the p-value is .05 or less. The successive mean elicited differences are increasing for both the gain and loss domain as shown in Table 10. Of 
these differences, three were significantly larger in the gain domain and two in the loss domain indicating increasingly more space between elicited outcomes and implying decreasing sensitivity to outcomes further from the reference point. This pattern implies that participants are generally risk averse in the gain domain and risk seeking in the loss domain. Since the lotterystyle questions are chained, this analysis is especially sensitive to outliers so all observations where the z-score of the mean elicited outcome was greater than 3.29 were excluded from the analysis. In addition, observations containing three or more strictly dominated responses in the utility sections are deemed illogical and excluded from the following analyses.

\section{Table 10 - Pooled Sampled T-Test of Successive Differences}

\begin{tabular}{|c|c|c|c|c|}
\hline & \multicolumn{2}{|c|}{ Gains $(\mathrm{n}=194)$} & \multicolumn{2}{c|}{ Losses $(\mathrm{n}=180)$} \\
\hline$j$ & $X_{j}$ & $X_{j}-X_{j-1}$ & $Y_{j}$ & $Y_{j}-Y_{j-1}$ \\
\hline 1 & 544 & 444 & -428 & -328 \\
\hline & $(398)$ & $(398)$ & $(381)$ & $(381)$ \\
\hline 2 & 912 & $369^{* * *}$ & -741 & -313 \\
\hline & $(660)$ & $(343)$ & $(619)$ & $(332)$ \\
\hline 3 & 1321 & $408^{*}$ & -1041 & -300 \\
\hline & $(970)$ & $(389)$ & $(867)$ & $(306)$ \\
\hline 4 & 1739 & 418 & -1363 & -322 \\
\hline & $(1332)$ & $(428)$ & $(1133)$ & $(325)$ \\
\hline 5 & 2242 & $503^{* * *}$ & -1722 & $-359^{* *}$ \\
\hline & $(1758)$ & $(570)$ & $(1443)$ & $(362)$ \\
\hline 6 & 2815 & $572^{* *}$ & -2137 & $-415^{* *}$ \\
\hline & $(2344)$ & $(763)$ & $(1792)$ & $(459)$ \\
\hline
\end{tabular}

The shape of the utility curve on an individual level is determined by normalizing the elicited outcomes such that all outcomes and their corresponding utility values lie within the unit square as shown in Figure 10. The elicited outcomes, $X_{j}$, are rescaled, $Z_{j}=\left(X_{j}-X_{0}\right) /\left(X_{6}-X_{0}\right)$, and the utility of those outcomes are set to $u\left(Z_{j}\right)=1 / 6$ for all $j=0, \ldots, 6$. Individual utility curves in the gain domain are classified as either convex, concave or linear depending if the area under the curve is less than, greater than, or equal to $1 / 2$, respectively. Since the elicited outcomes and corresponding utility values are transformed into positive numbers for the analysis, the opposite classification system is used for utility curvature in the loss domain. Figure 10 illustrates the area under the utility curve graphically. The frequencies of the classes of utility curves for gains and losses are listed in Table 11. In total, $48.5 \%$ of the participants exhibit concave utility functions in the gain domain and $46.2 \%$ exhibit convex utility functions in the loss domain. 
Figure 10 - Plot of the Area Under the Utility Curve

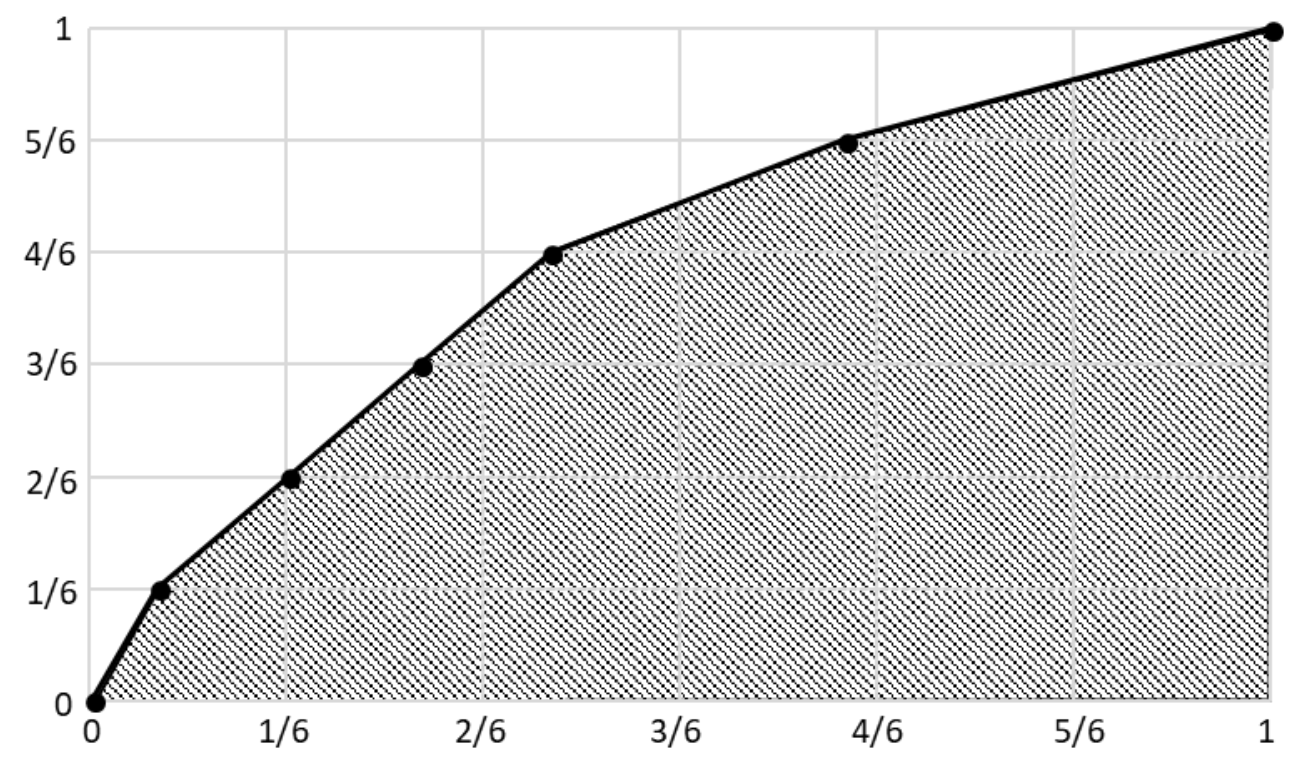

Table 11 - Non-Parametric Utility Curvature Frequencies

\begin{tabular}{|l|c|c|c|c|}
\hline & \multicolumn{2}{|c|}{ Gains $(\mathrm{n}=198)$} & \multicolumn{2}{c|}{ Losses $(\mathrm{n}=184)$} \\
\hline & Frequency & Percent & Frequency & Percent \\
\hline Concave & 96 & $48.5 \%$ & 61 & $33.2 \%$ \\
\hline Linear & 35 & $17.7 \%$ & 38 & $20.7 \%$ \\
\hline Convex & 67 & $33.8 \%$ & 85 & $46.2 \%$ \\
\hline Total & 198 & $100 \%$ & 184 & $100 \%$ \\
\hline
\end{tabular}

Table 12 shows the individual-level frequencies of the combination of utility curvatures across both domains and, in parentheses, the percentages of the sample that those frequencies represent. Most participants can be classified as having a utility curve that is convex (risk seeking) in the loss domain and concave (risk averse) in the gain domain. This is consistent with the law of diminishing sensitivity to outcomes and commonly reported empirical findings (Kahneman \& Tversky, 1979; Tversky \& Kahneman, 1992; Wakker \& Deneffe, 1996; Starmer, 2000; Booij, 2009).

Table 12 - Individual-Level Non-Parametric Utility Curvature

\begin{tabular}{|c|c|c|c|c|c|}
\hline & & \multicolumn{3}{|c|}{ Losses } & \\
\hline & & Concave & Linear & Convex & Total \\
\hline \multirow{3}{*}{ Gains } & Concave & $22(12 \%)$ & $7(4 \%)$ & $58(32 \%)$ & $87(49 \%)$ \\
\cline { 2 - 6 } & Linear & $4(2 \%)$ & $23(13 \%)$ & $2(1 \%)$ & $29(16 \%)$ \\
\cline { 2 - 6 } & Convex & $32(18 \%)$ & $8(4 \%)$ & $23(13 \%)$ & $63(35 \%)$ \\
\hline & Total & $58(32 \%)$ & $38(21 \%)$ & $83(46 \%)$ & $179(100 \%)$ \\
\hline
\end{tabular}




\subsubsection{Parametric Utility Analysis}

The parametric analysis provides for another way to interpret the data and allows for comparison with other studies reporting parametric utility estimates. The power parameter was calculated in both the gain and loss domains at an individual level for each of the 204 participants in the crosssectional dataset and for each of the 55 participants in all three trials in the longitudinal dataset. This was achieved by first normalizing the elicited outcomes, $X_{i}$, according to the method prescribed by Wakker and Deneffe (1996): $\left(X_{i}-X_{1}\right) /\left(X_{6}-X_{1}\right)$ for all $i=1, \ldots, 6$. The normalized outcomes and corresponding utility units were then transformed using natural logarithms and the power parameter calculated using an ordinary least squares linear regression. Table 13 lists the median and mean parameter estimates found by minimizing the sum of squared residuals in both the gain and loss domains.

\section{Table 13 - Sample Statistics for the Power Parameter in the Gain and Loss Domains}

\begin{tabular}{|l|c|c|}
\hline & $\theta^{+}(\mathrm{n}=174)$ & $\theta^{-}(\mathrm{n}=177)$ \\
\hline Median & .9450 & 1.0000 \\
\hline IQR & .2825 & .3800 \\
\hline Mean & .9541 & 1.0329 \\
\hline S.D. & .3335 & .4727 \\
\hline
\end{tabular}

Assuming the parametric form outlined in the parameter specification section, utility functions in the gain domain are classified as concave, convex or linear if the power parameter is less than, greater than or equal to one, respectively. Again, the opposite classification system is used for utility curvature in the loss domain. Tables 14 and 15 present the frequency and percentage of utility curvature classes for the parametric estimates.

Table 14 - Parametric Utility Curvature Frequencies

\begin{tabular}{|l|c|c|c|c|}
\hline & \multicolumn{2}{|c|}{ Gains $(\mathrm{n}=174)$} & \multicolumn{2}{c|}{ Losses $(\mathrm{n}=177)$} \\
\hline & Frequency & Percent & Frequency & Percent \\
\hline Concave & 99 & $56.9 \%$ & 57 & $32.2 \%$ \\
\hline Linear & 25 & $14.4 \%$ & 36 & $20.3 \%$ \\
\hline Convex & 50 & $28.7 \%$ & 84 & $47.5 \%$ \\
\hline Total & 174 & $100 \%$ & 177 & $100 \%$ \\
\hline
\end{tabular}


Table 15 - Individual-Level Parametric Utility Curvature

\begin{tabular}{|l|l|c|c|c|c|}
\hline & & \multicolumn{3}{|c|}{ Losses } & \\
\hline & & Concave & Linear & Convex & Total \\
\hline \multirow{3}{*}{ Gains } & Concave & $25(14 \%)$ & $6(3 \%)$ & $61(34 \%)$ & $92(51 \%)$ \\
\cline { 2 - 6 } & Linear & $2(1 \%)$ & $23(13 \%)$ & $2(1 \%)$ & $27(15 \%)$ \\
\cline { 2 - 6 } & Convex & $32(18 \%)$ & $6(3 \%)$ & $22(12 \%)$ & $60(34 \%)$ \\
\hline & Total & $59(33 \%)$ & $35(20 \%)$ & $85(47 \%)$ & $179(100 \%)$ \\
\hline
\end{tabular}

The parametric utility analysis finds median power parameter estimates of .95 and 1.00 for gains and losses, respectively. This is compared to the median empirical estimates of the power parameter from the reviewed literature which range from .49 to 1.01 for gains and from .72 to 1.06 for losses. Furthermore, the results of the total and individual-level parametric utility curvature assessments are consistent with the non-parametric results presented in tables 9 and 10. The parametric utility analysis shows that $34 \%$ percent of participants exhibited risk aversion in the gain domain and risk seeking in the loss domain. This is compared to $32 \%$ of participants exhibiting this pattern in the non-parametric analysis. This indicates that the power parameter is reasonably representative of the data and a good fit for the following analyses.

\subsection{Decision Weight Analysis}

\subsubsection{Non-Parametric Decision Weight Analysis}

The decision weight elicitation technique uses outcomes drawn from the utility elicitation questions to identify probabilities that make the participant indifferent between two prospects. For this reason, observations that contain three or more illogical responses in the first stage of the elicitation process are excluded from the analysis in addition to those found to contain three or more illogical responses in the decision weight elicitation questions. Of the initial decision weight dataset, 28 observations in the gain domain and 45 in the loss domain are identified as outliers and excluded from the following analyses. An additional observation from both the gain and loss domain is identified as exerting undue influence on the model and excluded from analysis. The final sample consists of 115 observations in the gain domain and 77 observations in the loss domain.

Assuming CPT, decision weights can be interpreted as the weighting attributed to the probabilities presented in the gamble. For example, a reported probability of .6 in question 18 corresponds to a decision weight of .5 which implies an over-weighting of probabilities in 50/50 gambles by .1 in the gain domain. The average and median values for each elicited probability 
and their corresponding decision weights are listed in Table 16. The median elicited probability values and corresponding decision weights are illustrated graphically in Figure 11 with a linear overlay for reference.

Table 16 - Summary Statistics for the Elicited Probabilities

\begin{tabular}{|c|c|c|c|c|c|c|c|c|}
\hline \multirow{2}{*}{$\begin{array}{c}\text { Corresponding } \\
\text { Decision Weight }\end{array}$} & \multicolumn{4}{|c|}{ Elicited Decision Weight $w^{+}(p)$} & \multicolumn{3}{c|}{ Elicited Decision Weight $w^{-}(p)$} \\
\cline { 2 - 9 } & Median & IQR & Mean & S.D. & Median & IQR & Mean & S.D. \\
\hline 16.67 & 30.00 & $20-40$ & 28.48 & 16.478 & 20.00 & $10-30$ & 23.70 & 18.615 \\
\hline 33.33 & 40.00 & $30-50$ & 40.11 & 17.034 & 30.00 & $20-43$ & 33.50 & 19.165 \\
\hline 50.00 & 50.00 & $40-60$ & 53.13 & 17.421 & 40.00 & $30-60$ & 45.51 & 19.926 \\
\hline 66.67 & 65.00 & $40-80$ & 64.26 & 16.852 & 50.00 & $42.5-70$ & 57.33 & 18.164 \\
\hline 83.33 & 75.00 & $65-90$ & 74.67 & 15.266 & 70.00 & $50-81.7$ & 69.15 & 16.993 \\
\hline
\end{tabular}

Figure 11 - Median Decision Weights with Linear Overlay
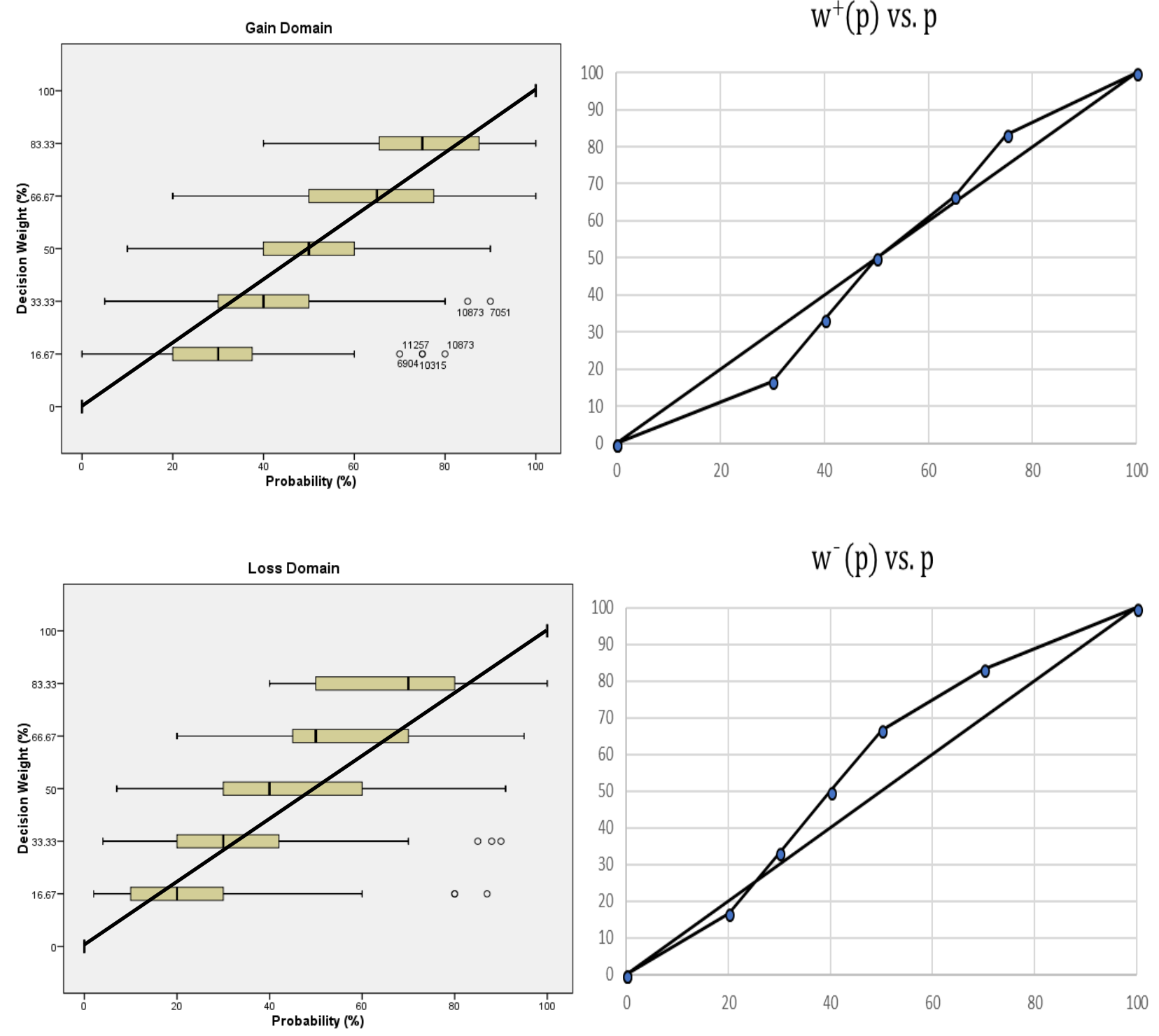
The point at which the probability weighting function crosses linearity in Figure 11 represents the point at which relative optimism and pessimism cancel out. This study finds that this point corresponds to between .5 and .67 in the gain domain and between .16 and .33 in the loss domain. This is comparable to studies conducted by Prelec (1998) and Quiggin (1981) who report linearity occurring at .33 and .5 , respectively.

The median probability weighting function follows an S-shaped pattern contrary to the most widely reported inverse $S$-shaped pattern and the psychological principle of diminishing sensitivity. There is, however, significant heterogeneity at the individual level and several recent studies have also found S-shaped median probability weighting functions (Alarié and Dionne, 2001; Humphrey and Verschoor, 2004; Harbaugh et al. 2002).

\section{Table 17 - Significant Differences from Linearity}

\begin{tabular}{|l|c|c|c|c|c|}
\hline $\mathrm{H}_{0}:$ & .1667 & .3333 & .5 & .6667 & .8333 \\
\hline$w^{+}(p) ;$ t-stat & $7.691^{* * *}$ & $4.264^{* * *}$ & $1.927^{*}$ & -1.533 & $-6.084^{* * *}$ \\
\hline$w^{-}(p) ;$ t-stat & $3.314^{* * *}$ & .075 & $-1.976^{*}$ & $-4.509^{* * *}$ & $-7.326^{* * *}$ \\
\hline
\end{tabular}

Table 17 presents a series of two-tailed paired samples t-tests $\left(\mathrm{H}_{0}: w^{ \pm}(p)=p\right)$ which reveals that the difference between the elicited decision weight and linearity is only mildly significant at a probability of .5 in both the gain and the loss domain. The difference is not significant at $w^{+}(p)=p=.6667$ and $w^{-}(p)=p=.3333$ in the gain and loss domain, respectively. This indicates that while both curves exhibit an S-shape pattern, the cross-over point is closer to the origin in the loss domain than in the gain domain. This is interpreted as participants exhibiting pessimistic behaviour over a greater range of probabilities in the loss domain than in the gain domain and therefore exhibit greater probabilistic risk aversion in the loss domain. Probabilistic risk seeking (aversion) can be characterized as areas of the probability weighting function that lie above (below) linearity in the gain domain and below (above) linearity in the loss domain.

\subsubsection{Parametric Decision Weight Analysis}

The decision weight parameters are calculated using the constrained nonlinear regression command in SPSS 23. This relies on a nonlinear least squares algorithm based on the LevenbergMarquardt algorithm to find the model parameters that best fit the data. This is an iterative process used to minimize the sum of squared errors of a non-linear function over a space of parameters with decision weights designated as the outcome variable and the elicited 
probabilities designated as explanatory variables. Table 18 presents the summary statistics for the decision weight parameters.

\section{Table 18 - Summary Statistics for the Decision Weight Parameters}

\begin{tabular}{|l|c|c|c|c|}
\hline & $\delta^{+}(114)$ & $\gamma^{+}(114)$ & $\delta^{-}(73)$ & $\gamma^{-}(73)$ \\
\hline Median & .98 & 1.38 & 1.77 & 1.34 \\
\hline IQR & $.49-1.84$ & $1.00-2.06$ & $.705-2.99$ & $.995-1.685$ \\
\hline Mean & 1.4629 & 1.6589 & 2.1892 & 1.4871 \\
\hline S.D. & 1.5502 & .9061 & 1.9449 & .6755 \\
\hline SSE Mean (S.D.) & \multicolumn{2}{|c|}{$.01654(.01867)$} & \multicolumn{2}{|c|}{$.01529(.01844)$} \\
\hline
\end{tabular}

Constraints are added such that $\delta^{+}, \delta^{-}>0$ and $\gamma^{+}, \gamma^{-}>0$ to ensure that the probability weighting function converges to the objective probabilities at zero and one. This constraint assumes that participants accept that if a zero probability is given, there is no chance of that event occurring and if a probability of 1 is given, the event is certain to occur. No other constraints were imposed on the function to ensure the independent and unique derivation of the model parameters.

The starting values for the parameter estimates were chosen using the median values presented in the seminal paper on the decision weight elicitation procedure used in this study: $\delta^{+}=.65$, $\delta^{-}=.84, \gamma^{+}=.60, \gamma^{-}=.65$ (Abdellaoui, 2000). This ensures that the first iteration is close to the estimates identified by the creator of the decision weight elicitation method used in this study and better ensures that the estimates reflect the global minimum.

Figure 12 illustrates the median probability weighting function with a linear overlay. The red gradients in Figure 12 a) and b) indicate areas where participants are probabilistic risk-seeking. This is interpreted as an over-weighting of the probability of obtaining the best-case scenario and under-weight of the probability of obtaining the worst-case scenario in figures a) and b), respectively. Conversely, the blue gradient in these figures indicate areas where participants are probabilistic risk-averse. 
a) Probability Weighting in Gains

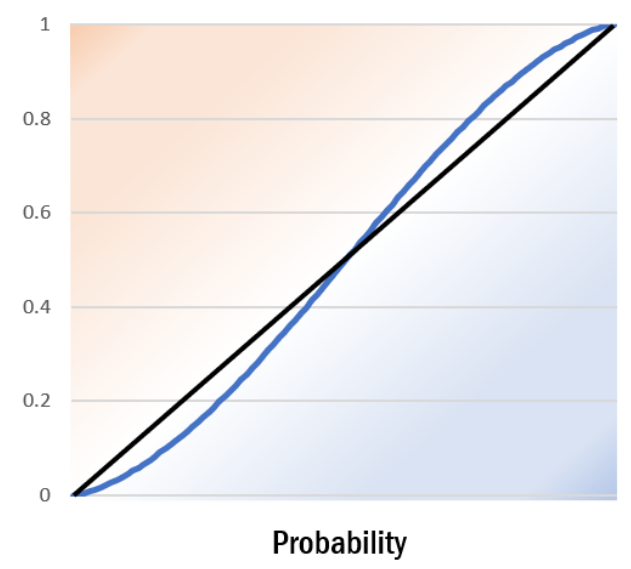

b) Probability Weighting in Losses

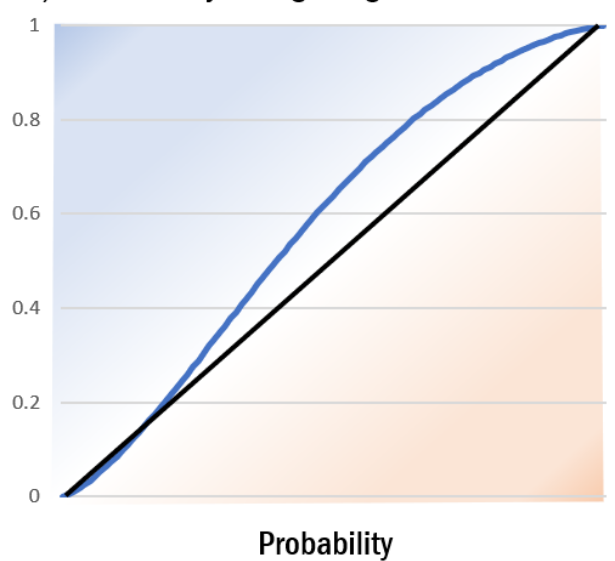

The shape of the median probability weighting functions is confirmed by the pattern of the median decision weights in the non-parametric analysis. Comparing the median curvature probability weighting parameters of 1.38 and 1.34 in the gain and loss domains, respectively, to previous median empirical estimates ranging from .44 to .83 in the gain domain and .53 to .84 in the loss domain, I find that the curvature parameter is significantly larger in the present study which leads to an S-shaped pattern observed in Figure 12. The median elevation probability weighting parameters of .98 and 1.77 for gains and losses, respectively, are more consistent with previous median empirical estimates ranging from .65 to .98 in the gain domain and .84 to 1.36 in the loss domain.

\subsection{Loss Aversion Analysis}

A loss aversion index is calculated for 203 cases. Of those, 59 were excluded because their answers led to inconclusive results and 54 were excluded because they provided three or more strictly dominated responses in the relevant sections. To be included in the loss aversion analysis participants must provide logical answers to both utility sections, the loss aversion section and have provided enough information in the decision weight sections to calculate their decision weight at a probability of .5 in both the gain and loss domains. Table 19 presents the summary statistics for the loss aversion index, $\lambda$, as defined in the 'Loss Aversion Parameter' section. 
Table 19 - Summary Statistics for the Loss Aversion Index

\begin{tabular}{|c|c|}
\hline & $\lambda(\mathrm{n}=90)$ \\
\hline Median & .9796 \\
\hline IQR & 1.2340 \\
\hline Mean & 1.4423 \\
\hline S.D. & 1.5360 \\
\hline
\end{tabular}

\subsection{Panel Effects}

The dataset for this study is separated into a strongly balanced panel dataset and a cross-sectional dataset. The panel dataset is used to determine the reliability of the elicited parameters and provide support for the findings presented in the cross-sectional analysis.

Both the panel and cross-sectional datasets are constructed from the same sample of participants, thought the criteria to be included in the panel dataset is more stringent. To be included in the panel dataset participants need to provide logical responses to at least one section in all three trials. The cross-sectional dataset, on the other hand, requires only that participants provide logical responses to one section in one of the three trials. The sample size in the panel dataset is therefore much smaller, $\mathrm{n}=55$, compared to the cross-sectional sample, $\mathrm{n}=204$. Since the power of the statistical tests on the panel dataset is significantly diminished, I rely on the cross-sectional analysis for a more comprehensive assessment and discussion of the effect of visceral factors on the parameters of interest.

The analyses performed on the panel data include a reliability analysis and a time- and entityfixed effects regression. A reliability analysis is performed to ensure the consistency of responses across trials and consists of Repeated Measures ANOVA (rmANOVA), MANOVA and a Related-Samples Friedman's Two-Way ANOVA. The results of this analysis found a significant difference in one trial of one of the seven model parameters. The results of the time- and entityfixed effects regression show that there is a significant panel effect in all seven parameters and at least weak statistical significance of one or more visceral factors on six of the seven parameters.

\subsubsection{Reliability Analysis}

A rmANOVA is conducted to test if there are significant within subject variations in the parameter estimates between each trial and subsequent trial. Outliers in the parameter estimates and residuals are identified as having standardized values greater than 3.29 or less than -3.29. Cases are excluded listwise to maintain a strongly balanced dataset and ensure the robustness of 
the tests (Lunney, 1970; Donaldson, 1968). The only observations that are excluded from the following analyses are those where there is evidence of comprehension issues. It is assumed that the participant did not fully understand the questions if they identified three or more strictly dominated responses as their indifference point.

The rmANOVA uses a non-orthogonal repeated contrast and a Bonferroni confidence interval adjustment. The final sample size and results of the analysis for each parameter are listed in tables 20 and 21 below. I find only one significant difference in parameter estimates between trials indicating that there are no systematic effects that might bias the results.

\section{Table 20 - Variables of Interest and Tests of Within-Subject Changes}

\begin{tabular}{|c|c|c|c|c|}
\hline Variable & Trial & Mean (s.d.) & Within-Subject Change & Significant differences \\
\hline \multirow[t]{3}{*}{$\theta^{+}(n=46)$} & 1 & $.8724(.2283)$ & \multirow{3}{*}{$\begin{array}{l}\text { F-Stat }=4.299, p=.021 \\
d f=1.739 / 78.256\end{array}$} & \multirow[t]{3}{*}{$2<3$} \\
\hline & 2 & $.8867(.2256)$ & & \\
\hline & 3 & $1.0167(.3791)$ & & \\
\hline \multirow[t]{3}{*}{$\boldsymbol{\theta}^{-}(n=40)$} & 1 & $.9200(.2726)$ & \multirow{3}{*}{$\begin{array}{l}\text { F-Stat }=.104, p=.438 \\
d f=2 / 78\end{array}$} & \multirow[t]{3}{*}{ None } \\
\hline & 2 & $.8993(.3194)$ & & \\
\hline & 3 & $.9695(.2746)$ & & \\
\hline \multirow{4}{*}{$\boldsymbol{\delta}^{+}(n=38)$} & 1 & & \multirow{4}{*}{$\begin{array}{l}\text { F-Stat }=\mathbf{3 . 5 0 9 ,} p=.433 \\
d f=1.418 / 52.468\end{array}$} & \multirow{4}{*}{ None } \\
\hline & 1 & $1.5208(1.2855)$ & & \\
\hline & 2 & $1.9432(2.2969)$ & & \\
\hline & 3 & $1.6632(1.4215)$ & & \\
\hline \multirow[t]{3}{*}{$\gamma^{+}(n=38)$} & 1 & $1.7621(.8783)$ & \multirow{3}{*}{$\begin{array}{l}\text { F-Stat }=.344, p=.710 \\
d f=2 / 74\end{array}$} & \multirow[t]{3}{*}{ None } \\
\hline & 2 & $1.7139(.7028)$ & & \\
\hline & 3 & $1.8445(.9444)$ & & \\
\hline \multirow[t]{3}{*}{$\delta^{-}(n=27)$} & 1 & 2.2459 (1.9473) & \multirow{3}{*}{$\begin{array}{l}\text { F-Stat }=20.227, p=.322 \\
d f=1.616 / 42.027\end{array}$} & \multirow[t]{3}{*}{ None } \\
\hline & 2 & $2.6978(2.9440)$ & & \\
\hline & 3 & $3.4570(5.0316)$ & & \\
\hline \multirow[t]{3}{*}{$\gamma^{-}(\mathrm{n}=23)$} & 1 & $1.4230(.6816)$ & \multirow{3}{*}{$\begin{array}{l}\text { F-Stat }=.319, p=.728 \\
d f=2 / 44\end{array}$} & \multirow[t]{3}{*}{ None } \\
\hline & 2 & $1.5448(.8292)$ & & \\
\hline & 3 & $1.5404(.7157)$ & & \\
\hline \multirow[t]{3}{*}{$\lambda(n=21)$} & 1 & $1.0298(.9076)$ & \multirow{3}{*}{$\begin{array}{l}\text { F-Stat }=.354, p=.704 \\
d f=2 / 40\end{array}$} & \multirow[t]{3}{*}{ None } \\
\hline & 2 & $.9602(.6288)$ & & \\
\hline & 3 & .8581 (.6189) & & \\
\hline
\end{tabular}

Table 21 - Tests of Within-Subjects Changes Over the Three Trials

\begin{tabular}{|l|c|c|c|c|c|c|c|}
\hline & $\begin{array}{c}\boldsymbol{\theta}^{+} \\
(\mathrm{n}=46)\end{array}$ & $\begin{array}{c}\boldsymbol{\theta}^{-} \\
(\mathrm{n}=40)\end{array}$ & $\begin{array}{c}\boldsymbol{\delta}^{+} \\
(\mathrm{n}=38)\end{array}$ & $\begin{array}{c}\boldsymbol{\gamma}^{+} \\
(\mathrm{n}=38)\end{array}$ & $\begin{array}{c}\boldsymbol{\delta}^{-} \\
(\mathrm{n}=27)\end{array}$ & $\begin{array}{c}\boldsymbol{\gamma}^{-} \\
(\mathrm{n}=23)\end{array}$ & $\boldsymbol{\lambda}(\mathrm{n}=21)$ \\
\hline Tests & & & & & & & \\
\hline Pillai's trace - F-stat (sig.) & 3.379 & .722 & .486 & .331 & 1.312 & .364 & .462 \\
& $(.043)$ & $(.492)$ & $(.619)$ & $(.721)$ & $(.287)$ & $(.699)$ & $(.637)$ \\
\hline Sphericity Assumed - F-stat (sig.) & 4.299 & .104 & .754 & .344 & 1.130 & .319 & .354 \\
& $(.016)$ & $\mathbf{( . 4 3 8 )}$ & $(.474)$ & $\mathbf{( . 7 1 0 )}$ & $(.331)$ & $\mathbf{( . 7 2 8 )}$ & $(.704)$ \\
\hline
\end{tabular}




\begin{tabular}{|c|c|c|c|c|c|c|c|c|}
\hline \multicolumn{2}{|c|}{ Greenhouse-Geisser - F-stat (sig.) } & $\begin{array}{l}4.299 \\
(.023)\end{array}$ & $\begin{array}{l}.104 \\
(.432)\end{array}$ & $\begin{array}{c}.754 \\
(.433)\end{array}$ & $\begin{array}{c}.344 \\
(.708)\end{array}$ & $\begin{array}{l}1.130 \\
(.320)\end{array}$ & $\begin{array}{l}.319 \\
(.719)\end{array}$ & $\begin{array}{c}.354 \\
(.642)\end{array}$ \\
\hline \multicolumn{2}{|c|}{ Huynh-Feldt - F-stat (sig.) } & $\begin{array}{l}4.299 \\
(.021)\end{array}$ & $\begin{array}{c}.104 \\
(.437)\end{array}$ & $\begin{array}{c}.754 \\
(.436)\end{array}$ & $\begin{array}{c}.344 \\
(.710)\end{array}$ & $\begin{array}{l}1.130 \\
(.322)\end{array}$ & $\begin{array}{c}.319 \\
(.728)\end{array}$ & $\begin{array}{c}.354 \\
(.655)\end{array}$ \\
\hline \multicolumn{2}{|c|}{ Lower-bound - F-stat (sig.) } & $\begin{array}{l}4.299 \\
(.044)\end{array}$ & $\begin{array}{c}.104 \\
(.367)\end{array}$ & $\begin{array}{c}.754 \\
(.391)\end{array}$ & $\begin{array}{c}.344 \\
(.561)\end{array}$ & $\begin{array}{l}1.130 \\
(.298)\end{array}$ & $\begin{array}{c}.319 \\
(.578)\end{array}$ & $\begin{array}{c}.354 \\
(.559)\end{array}$ \\
\hline \multicolumn{9}{|l|}{ Assumptions } \\
\hline \multicolumn{2}{|c|}{ Mauchly's Test of Sphericity (sig.) } & $\begin{array}{c}.810 \\
(.010)\end{array}$ & $\begin{array}{c}.939 \\
(.300)\end{array}$ & $\begin{array}{c}.590 \\
(<.001)\end{array}$ & $\begin{array}{c}.994 \\
(.896)\end{array}$ & $\begin{array}{c}.700 \\
(.012)\end{array}$ & $\begin{array}{c}.954 \\
(.610)\end{array}$ & $\begin{array}{c}.659 \\
(.019)\end{array}$ \\
\hline \multirow{3}{*}{$\begin{array}{l}\text { Shapiro-Wilk } \\
\text { (sig.) }\end{array}$} & Std. res. 1 & .499 & .321 & .002 & .004 & $<.001$ & .019 & .003 \\
\hline & Std. res. 2 & .326 & .001 & .000 & .073 & $<.001$ & $<.001$ & .325 \\
\hline & Std. res. 3 & .041 & .092 & .003 & .002 & $<.001$ & .019 & .244 \\
\hline
\end{tabular}

The tests of within subject contrasts revealed a significant difference between the power parameters elicited in the second and third trials. The results of the analysis showed violations of multivariate normal distribution though the F-statistic has been shown to control the type 1 error rate well under conditions of skewness, kurtosis and non-normal distributions in strongly balanced datasets with more than 20 degrees of freedom (Lunney, 1970; Donaldson, 1968).

The findings from the rmANOVA are confirmed with Pillai's trace test statistic and a relatedsamples Friedman's two-way ANOVA by ranks presented in tables 19 and 20, respectively. The related-samples Friedman's two-way ANOVA by ranks has a chi-squared distribution with 2 degrees of freedom and a sample size of 55. This test was run on each model parameter with all pairwise comparisons amongst trials. Findings indicate that there is a significant difference between the first and third trials of the power parameter estimates in the gain domain, F-Stat = $.473, \mathrm{p}=.013$.

\section{Table 22 - Related-Samples Friedman's Two-Way ANOVA, and All Pairwise}

\section{Comparisons.}

\begin{tabular}{|c|c|c|c|c|c|c|c|}
\hline & $\theta^{+}$ & $\boldsymbol{\theta}^{-}$ & $\delta^{+}$ & $\gamma^{+}$ & $\boldsymbol{\delta}^{-}$ & $\gamma^{-}$ & $\lambda$ \\
\hline \multicolumn{8}{|l|}{ Within-Subject Change } \\
\hline Friedman's Test - $F_{r}$ (sig.) & $\begin{array}{l}6.624 \\
(.036)\end{array}$ & $\begin{array}{l}4.010 \\
(.135)\end{array}$ & $\begin{array}{l}1.710 \\
(.425)\end{array}$ & $\begin{array}{l}1.231 \\
(.540)\end{array}$ & $\begin{array}{l}2.625 \\
(.269)\end{array}$ & $\begin{array}{l}1.279 \\
(.528)\end{array}$ & $\begin{array}{l}.412 \\
(.814)\end{array}$ \\
\hline
\end{tabular}

\subsubsection{Fixed Effects Regression}

Fixed effects models are often used in economics to infer causality in natural experiments by controlling for unobserved heterogeneity amongst entities and over time. In the fixed effects model, entity- and/or time-invariant confounds are absorbed by the intercept and the coefficient estimates are immune to omitted variable bias resulting from unobserved variables that are either constant over time or across entities. They cannot, however, control for omitted variables that 
vary across entities and over time such as emotions and other visceral factors which must be included as regressors.

Fixed effects regressions can be classified as either entity-fixed effects regressions or timefixed effects regressions. Entity-fixed effects regressions control for variables that differ amongst participants but remain constant over the data collection period. Some examples of entity-fixed effects include cultural values, certain contextual effects, personal experiences, weight, diet, demographic factors and personality traits. Time-fixed effects regressions control for variables that affect all participants in the sample but that change over the data collection period. Examples of time-fixed effects include government policies (tax returns, holidays), the weather and university schedules such as exam week. The logic behind entity-fixed effects regressions is that if something did not change for a given participant, then it could not have not caused a change in that participant's behaviour. The same logic can be applied to variables that change over time but are constant across entities.

Time- and entity-fixed effects models can be estimated using the following two approaches. The first is referred to as the binary variable specification,

$$
Y_{i t}=\beta_{0}+\beta_{1} X_{1, i t}+\cdots+\beta_{k} X_{k, i t}+F_{i}+T_{t}+\varepsilon_{i t}
$$

where:

- $Y_{i t}$ is the dependent variable for entity $i$ at time $t$,

- $\beta_{1}, \ldots, \beta_{k}$ are the coefficients for $k$ independent variables,

- $X_{1, i t}, \ldots, X_{k, i t}$ represent $k$ independent variables for entity $i$ at time $t$,

- $F_{i}(i=2, \ldots, n)$ is the unknown entity-specific intercept for $n-1$ entities,

- $T_{t}(t=2, \ldots, m)$ is the unknown time-specific intercept for $m-1$ time periods,

- $\varepsilon_{i t}$ is the error term for entity $i$ at time $t$.

This model is estimated by creating $(n-1)+(m-1)$ dummy variables for each entity and time period, respectfully, and regressing $Y_{i t}$ on all of the independent variables, $X_{k, i t}$, and the dummy variables, $F_{i}$ and $T_{t}$.

An equivalent approach is known as the entity-demeaned specification. This is a two-step process where the entity- and/or time-specific average for each variable is subtracted from each of the respective variables prior to running the regression using the entity-demeaned variables. 
The entity-demeaned specification provides identical OLS estimates as the binary variable specification but requires fewer parameters and is less computationally intensive.

The coefficients in the following time- and entity-fixed effects regression are estimated using a combination of the two specifications. The entity-demeaned OLS algorithm in Stata/SE 13.0 was used to control for entity-fixed effects and two binary variables were created to control for time-fixed effects:

$$
Y_{i t}-\bar{Y}_{i}=\beta_{0}+\beta_{1}\left(X_{1, i t}-\bar{X}_{1, i}\right)+\cdots+\beta_{k}\left(X_{k, i t}-\bar{X}_{k, i}\right)+T_{t}+\varepsilon_{i t}
$$

where,

- $Y_{i t}-\bar{Y}_{i}$ is the entity-demeaned dependent variable for entity $i$ at time $t$,

- $X_{k, i t}-\bar{X}_{k, i}$ is the $k^{t h}$ entity-demeaned independent variable for entity $i$ at time $t$,

- $T_{t}(t=2, \ldots, m)$ is the unknown time-specific intercept for $m-1$ time periods,

- $\varepsilon_{i t}$ is the error term for entity $i$ at time $t$.

The results of seven time- and entity-fixed effects regressions are presented in Table 23 along with model diagnostics and tests of the underlying assumptions.

Table 23 - Time- and Entity-Fixed Effects Regressions

\begin{tabular}{|l|c|c|c|c|c|c|c|}
\hline & $\begin{array}{c}\boldsymbol{\theta}^{+} \\
(\mathrm{n}=46)\end{array}$ & $\begin{array}{c}\boldsymbol{\theta}^{-} \\
(\mathrm{n}=41)\end{array}$ & $\begin{array}{c}\boldsymbol{\delta}^{+} \\
(\mathrm{n}=39)\end{array}$ & $\begin{array}{c}\boldsymbol{\gamma}^{+} \\
(\mathrm{n}=38)\end{array}$ & $\begin{array}{c}\boldsymbol{\delta}^{-} \\
(\mathrm{n}=27)\end{array}$ & $\begin{array}{c}\boldsymbol{\gamma}^{-} \\
(\mathrm{n}=23)\end{array}$ & $\begin{array}{c}\lambda \\
(\mathrm{n}=43)\end{array}$ \\
\hline Variables & & & & & & & \\
\hline Constant & $\mathbf{9 6 4 2}$ & $\mathbf{1 . 1 3 4 4}$ & $\mathbf{2 . 0 1 4 6}$ & $\mathbf{2 . 0 4 7 8}$ & .8888 & .9923 & $\mathbf{1 . 4 8 1 5}$ \\
& $(<.001)$ & $(<.001)$ & $(.057)$ & $(<.001)$ & $(.592)$ & $(.002)$ & $(<.001)$ \\
\hline Angry & .0023 & -.0186 & .0868 & $\mathbf{. 1 6 7 3}$ & -.1138 & -.0444 & -.1255 \\
& $(.915)$ & $(.420)$ & $(.562)$ & $\mathbf{( . 0 1 2 )}$ & $(.634)$ & $(.644)$ & $(.197)$ \\
\hline Sad & -.0056 & -.0148 & .0376 & -.0378 & .1530 & .0618 & -.1255 \\
& $(.690)$ & $(.434)$ & $(.559)$ & $(.318)$ & $(.302)$ & $(.035)$ & $(.274)$ \\
\hline Happy & -.0385 & -.0252 & .2201 & -.0817 & .2419 & .0605 & -.0530 \\
& $(.023)$ & $(.131)$ & $(.157)$ & $(.063)$ & $(.345)$ & $(.206)$ & $(.374)$ \\
\hline Anxious & .0050 & .0148 & .1126 & .0117 & .3556 & .0373 & -.0347 \\
& $(.672)$ & $(.409)$ & $(.240)$ & $(.775)$ & $(.070)$ & $(.414)$ & $(.575)$ \\
\hline Hungry & .0018 & -.0113 & -.0528 & .0429 & .0550 & .0657 & -.0124 \\
& $(.842)$ & $(.316)$ & $(.538)$ & $(.226)$ & $(.792)$ & $(.114)$ & $(.704)$ \\
\hline Energetic & .0175 & .0106 & -.3743 & -.0084 & -.1825 & -.0524 & .0519 \\
& $(.275)$ & $(.336)$ & $(.005)$ & $(.860)$ & $(.514)$ & $(.284)$ & $(.449)$ \\
\hline Tired & .0003 & -.0091 & -.0957 & -.0293 & .0392 & .0017 & .0519 \\
& $(.980)$ & $(.396)$ & $(.247)$ & $(.424)$ & $(.905)$ & $(.967)$ & $(.076)$ \\
\hline Stressed & .0023 & -.0155 & -.1151 & -.0427 & -.1114 & -.0232 & .0063 \\
& $(.863)$ & $(.409)$ & $(.261)$ & $(.260)$ & $(.546)$ & $(.697)$ & $(.922)$ \\
\hline Trial 2 & .0039 & -.0163 & .2319 & .1024 & .2298 & .1461 & .0576 \\
& $(.934)$ & $(.742)$ & $(.565)$ & $(.574)$ & $(.731)$ & $(.482)$ & $(.844)$ \\
\hline Trial 3 & .1273 & .0309 & .0734 & .1490 & 1.1212 & .2350 & -.0686 \\
& $(.0133)$ & $(.547)$ & $(.802)$ & $(.399)$ & $(.220)$ & $(.235)$ & $(.779)$ \\
\hline
\end{tabular}




\begin{tabular}{|c|c|c|c|c|c|c|c|}
\hline & & & & & & & \\
\hline \multicolumn{8}{|l|}{ Model Diagnostics } \\
\hline $\mathrm{R}^{2}$ : within & .1683 & .1611 & .2203 & .1710 & .1003 & .1736 & .1495 \\
\hline F-test - sig. & .1589 & $<.001$ & .1950 & .0868 & .3563 & .3274 & .0979 \\
\hline $\operatorname{Corr}\left(u_{i}, X_{i}\right)$ & -.1603 & -.4801 & -.3252 & -.2702 & -.2677 & -.2223 & -.1841 \\
\hline rho & .3709 & .5227 & .4696 & .5061 & .4339 & .5046 & .3213 \\
\hline \multicolumn{8}{|l|}{ Assumptions } \\
\hline $\begin{array}{l}\text { Wooldridge test } \\
\text { (sig.) }\end{array}$ & $\begin{array}{c}.628 \\
(.4322) \\
\end{array}$ & $\begin{array}{c}.763 \\
(.3877) \\
\end{array}$ & $\begin{array}{c}5.380 \\
(.0258) \\
\end{array}$ & $\begin{array}{r}1.308 \\
(.2601) \\
\end{array}$ & $\begin{array}{c}6.960 \\
(.0139) \\
\end{array}$ & $\begin{array}{r}2.685 \\
(.1155) \\
\end{array}$ & $\begin{array}{l}10.213 \\
(.0045) \\
\end{array}$ \\
\hline $\begin{array}{l}\text { Modified Wald test } \\
\text { (sig.) }\end{array}$ & $\begin{array}{l}1.8 \mathrm{e}+6 \\
(<.001) \\
\end{array}$ & $\begin{array}{l}55699 \\
(<.001) \\
\end{array}$ & $\begin{array}{l}1.3 e+5 \\
(<.001)\end{array}$ & $\begin{array}{l}26397 \\
(<.001) \\
\end{array}$ & $\begin{array}{l}1.2 \mathrm{e}+5 \\
(<.001) \\
\end{array}$ & $\begin{array}{c}9869 \\
(<.001) \\
\end{array}$ & $\begin{array}{c}1.0 \mathrm{e}+33 \\
(<.001) \\
\end{array}$ \\
\hline \multicolumn{8}{|l|}{ Model Selection } \\
\hline Hausman test (sig.) & $\begin{array}{c}3.39 \\
(.9706) \\
\end{array}$ & $\begin{array}{c}11.75 \\
(.3023) \\
\end{array}$ & $\begin{array}{c}13.73 \\
(.1855) \\
\end{array}$ & $\begin{array}{c}12.04 \\
(.2824) \\
\end{array}$ & $\begin{array}{c}5.59 \\
(.8487) \\
\end{array}$ & $\begin{array}{r}13.99 \\
(.1733) \\
\end{array}$ & $\begin{array}{c}3.88 \\
(.9526) \\
\end{array}$ \\
\hline $\begin{array}{l}\text { Breusch-Pagan LM } \\
\text { - chibar(01) (Prob > } \\
\text { chibar) }\end{array}$ & $\begin{array}{c}4.61 \\
(.0159)\end{array}$ & $\begin{array}{c}3.92 \\
(.0239)\end{array}$ & $\begin{array}{c}6.69 \\
(.0049)\end{array}$ & $\begin{array}{c}8.04 \\
(.0023)\end{array}$ & $\begin{array}{c}3.02 \\
(.0410)\end{array}$ & $\begin{array}{c}5.79 \\
(.008)\end{array}$ & $\begin{array}{l}<.001 \\
(1.00)\end{array}$ \\
\hline
\end{tabular}

* The significance tests of the parameters coefficients in the models that exhibit groupwise heteroscedasticity are performed using the heteroscedasticity-consistent Huber/White (sandwich) estimation procedure.

** The significance tests of the parameter coefficients in models that exhibit groupwise heteroscedasticity and serial correlation are based on clustered standard errors.

The results of this analysis provide strong evidence supporting the effect of visceral factors on decision making under risk in four of the seven parameters that characterize risk preference under CPT and weak evidence supporting their effect on an additional two parameters. Given the relatively small sample used in this analysis, I note strong and weak support levels characterized by p-values less than .05 and .1, respectively.

This analysis also finds support for the inclusion of demographic factors in cross-sectional analyses of the determinants of risk preference. The Breusch-Pagan Lagrange Multiplier (LM) determines if there is a significant difference between a random effects regression and a simple OLS regression by testing the hypothesis that there is no significant difference across groups. I reject this hypothesis in all seven parameters indicating that there is a significant panel effect in all models. This implies that the effect of the visceral factors on the parameters of interest are not uniform and that it is necessary to include entity-specific intercepts. In addition, the rho values presented in Table 23 indicate between $32 \%$ and 52\% of the variance is due to differences across panels, or rather the entity-fixed effects. These findings support the need to include demographic factors in my final model to further classify participants and allow for the intercepts of participants to differ. 
It is also worth mentioning that the Hausman tests are not significant for any of the models which implies that a random effects model is more appropriate than a fixed effects model. The Hausman test determines if there are any systematic differences in the estimated coefficients, or rather that the unique errors are not correlated with the regressors. One assumption of the random effects model, however, is that the unobserved variables are statistically independent of the observed variables. According to the literature and previous analyses, certain personality traits are strongly correlated with visceral factors. This is supported, in part, by an average correlation of -.27 between the errors and the regressors in the current analysis. If there is a correlation between the regressors and unobserved variables, then the random effects estimator is inconsistent, and the fixed effects model is more appropriate.

\subsection{Model Development}

To explore the relationship between visceral factors and the magnitude of the parameters that characterize risk preference under CPT, I assume the following relationship:

$$
\varphi_{i}=\beta_{0}+\beta_{1} \chi_{1, i}+\cdots+\beta_{k} \chi_{k, i}+\varepsilon_{i}
$$

where the dependent variable, $\varphi$, represents each of the parameters that characterize risk preference under prospect theory $\left(\theta^{+}, \theta^{-}, \delta^{+}, \delta^{-}, \gamma^{+}, \gamma^{-}\right.$, and $\left.\lambda\right)$ for entity $i$ and the independent variables, $\chi_{i}$, represent all of the demographic variables, personality traits, visceral factors and relevant interaction terms. All model parameter estimates, $\varphi$, are assumed unique, separable and logically independent factors of risk preference.

\subsubsection{Dimension Reduction}

A Non-Linear Principle Components Analysis (NLPCA) was performed to explore opportunities to reduce the number of variables in the following analyses. This analysis is an alternative to Principle Components Analysis that uses the built-in optimal scaling feature in SPSS 23 to analyse non-linearly related variables with different types of measurement levels (i.e. numerical, categorical, etc.). Optimal scaling works by transforming categories of variables with nominal or ordinal scales to numeric values based on the centroid model with restrictions imposed by the researcher. Categories are assigned values in the principle component space by optimizing the values according to the model that it is trying to fit. 
An analysis level set by the researcher determines the amount of freedom allowed when transforming variables. The numeric level is the most restrictive and will not reveal nonlinear or nonmonotonic relationships between variables. This is followed by the ordinal scale which will reveal nonlinear relationships, but not nonmonotonic relationships between variables. Lastly, the nominal level is least restrictive and will reveal non-linear, non-monotonic relationships. The analysis levels for this analysis were set to the measurement levels in the study with variables such as age and gross wealth set to numeric, categorical variables such as gender and occupation set to nominal, and ordinal variables such as education, employment status, visceral factors and personality traits set to spline ordinal. The spline feature in optimal scaling smooths the curve between data points and is the preferred setting when investigating nonlinear relationships between variables. The smoothness of the resulting curve is defined by the number of interior knots set by the researcher.

The dimensions or components in this analysis are explanatory constructs that reflect clusters of variables that correlate highly with one another. Though I cannot use NLPCA to infer the existence of underlying constructs, they are useful in understanding the relationships amongst variables used in the following analyses.

An initial trial revealed 'Investment Experience' and 'Number of Dependents' with Variance Accounted For (VAF) scores of only .381 and .459, respectively, and were subsequently excluded from the analysis. 'Married' and 'Combined Income' were also excluded due to an insufficient number of observations. 20 observations were also found to have object scores (component scores) less than -3.5 or greater than 3.5 and were excluded to ensure proper variable fit.

The final model presented in Table 24 includes 14 components which account for $88.79 \%$ of the variation in the data. This model was constructed using an iterative process based on each component's Cronbach's alpha and Kaiser's criterion of Eigenvalues greater than 1 (Kaiser, 1960; Jolliffe, 1972; 1986). The verimax orthogonal rotation method with Kaiser's normalization was used following an analysis of the component correlations using the 'direct Oblimin' rotation method. The Cronbach's alpha is also listed but should not be used as a measure of internal reliability for mixed data since it is sensitive to the number of items in a scale. Based on the 
literature, component loadings greater than .3 are significant given the sample size (Stevens, 2002) and are presented in bold.

\section{Table 24 - Rotated Component Loadings from the NLPCA}

\begin{tabular}{|c|c|c|c|c|c|c|c|c|c|c|c|c|c|c|}
\hline$(n=269)$ & \multicolumn{14}{|c|}{ Dimension } \\
\hline & 1 & 2 & 3 & 4 & 5 & 6 & 7 & 8 & 9 & 10 & 11 & 12 & 13 & 14 \\
\hline Angry & .896 & .183 & .017 & .014 & -.052 & .014 & -.087 & .014 & .051 & .021 & -.034 & -.108 & .079 & -.008 \\
\hline Sad & .868 & .148 & -.037 & .029 & .043 & .152 & .081 & -.028 & .035 & .023 & -.084 & .051 & -.035 & .012 \\
\hline Anxious & .656 & .377 & .034 & .123 & .042 & .299 & .226 & -.036 & -.129 & -.062 & -.064 & .142 & -.060 & -.038 \\
\hline Energetic & .184 & .891 & .055 & -.125 & -.060 & .017 & -.076 & -.033 & -.050 & .057 & .001 & -.007 & .009 & -.015 \\
\hline Happy & .147 & .836 & .007 & -.085 & .051 & .263 & -.067 & -.060 & .018 & .026 & .016 & -.119 & .017 & .048 \\
\hline Hungry & .331 & .704 & .057 & -.006 & .063 & .184 & .155 & .171 & .014 & -.028 & -.025 & -.067 & .141 & -.058 \\
\hline Net Wealth & -.010 & .033 & .929 & -.052 & .087 & .030 & .002 & -.041 & .106 & -.006 & .005 & -.077 & .020 & -.062 \\
\hline Gross Wealth & .002 & .054 & .903 & .058 & .172 & .001 & -.023 & -.004 & .133 & -.110 & .087 & .029 & .037 & -.023 \\
\hline Openness & .000 & -.018 & -.001 & .932 & -.036 & -.060 & .098 & -.038 & .037 & .014 & -.074 & .079 & .015 & .107 \\
\hline Extraversion & -.093 & .161 & .001 & -.911 & .025 & .000 & -.034 & -.084 & -.023 & -.012 & .037 & .020 & .050 & .073 \\
\hline Empl_Status & .020 & -.080 & .077 & -.015 & .891 & .171 & .025 & .054 & .094 & .055 & .002 & .033 & .049 & -.100 \\
\hline Occupation & -.014 & -.220 & -.311 & .088 & -.705 & .185 & .181 & .010 & .240 & .119 & .091 & -.134 & .200 & -.117 \\
\hline Yearly Income & .029 & .035 & .398 & -.019 & .509 & -.022 & .105 & -.120 & .392 & -.229 & .307 & -.108 & .045 & .045 \\
\hline Tired & .221 & .307 & .032 & -.091 & .030 & .836 & -.035 & .042 & .001 & .115 & -.076 & .000 & -.120 & .013 \\
\hline Stressed & .513 & .324 & -.008 & .021 & .137 & .608 & .171 & -.110 & -.112 & -.018 & -.093 & .093 & .035 & .006 \\
\hline Neuroticism & .102 & -.023 & -.013 & .130 & -.037 & .027 & .931 & -.092 & -.008 & .035 & -.118 & .027 & -.186 & -.011 \\
\hline Agreeableness & -.037 & .016 & -.052 & .042 & .022 & -.006 & -.086 & 1.001 & -.038 & -.031 & -.026 & -.014 & .022 & .062 \\
\hline Age & .008 & -.029 & .274 & .069 & .024 & -.046 & -.026 & -.033 & .860 & -.043 & -.062 & .263 & -.012 & -.022 \\
\hline Head of House & .010 & .052 & -.114 & .024 & -.037 & .076 & .033 & -.033 & -.050 & .968 & .004 & -.012 & .042 & .110 \\
\hline Conscientiousness & -.148 & -.007 & .088 & -.110 & .000 & -.091 & -.122 & -.023 & -.038 & .009 & .941 & .096 & .018 & -.040 \\
\hline Education & .019 & -.133 & -.061 & .056 & .070 & .035 & .028 & -.015 & .205 & -.010 & .093 & .930 & .055 & -.007 \\
\hline Gender & .025 & .099 & .056 & -.034 & -.028 & -.082 & -.178 & .023 & -.004 & .042 & .018 & .054 & .950 & .068 \\
\hline \# Ppl House & -.012 & -.014 & -.070 & .032 & -.030 & .011 & -.011 & .061 & -.015 & .107 & -.035 & -.007 & .064 & .982 \\
\hline Eigenvalues & 2.507 & 2.504 & 2.061 & 1.804 & 1.635 & 1.379 & 1.108 & 1.094 & 1.074 & 1.059 & 1.059 & 1.053 & 1.043 & 1.042 \\
\hline VAF (\%) & 10.9 & 10.87 & 8.96 & 7.84 & 7.11 & 6.00 & 4.82 & 4.76 & 4.67 & 4.60 & 4.60 & 4.58 & 4.53 & 4.53 \\
\hline Cronbach's alpha & .755 & .750 & .643 & .513 & .541 & .659 & .353 & .118 & .365 & .251 & .307 & .187 & .168 & .105 \\
\hline
\end{tabular}

\subsubsection{Variable Selection}

Two-way interaction variables were created based on bivariate correlations of independent

variables and visual inspections of independent and dependent variable scatter plot diagrams at

different levels of interacting variables. A total of 34 two-way interaction variables were created and explored for significant effects on each of the model parameters.

All participants reported 20+ years to retirement except for one who reported 10-19 years and two who reported 0-9 years. For this reason, 'Years to Retirement' is excluded as an independent variable. Additionally, three pairs of variables 'Age' \& 'Age squared', 'Net Wealth' \& 'Gross Wealth' and 'Openness' and 'Extraversion' are highly correlated. The three variables that showed the least significance on each of the model parameters, 'Age squared', 'Net Wealth' and 'Openness', are removed to avoid issues with multicollinearity. 
A backwards elimination is performed with stepping method criteria set to remove variables based on a probability value of the test statistic ranging from .1 to .5 and reinclude variables with a test statistic ranging from .05 to .3 . A stepwise regression is also conducted on the same dataset with the stepping method entry criteria ranging from .3 to .5 and removal value of .5 . Table 25 lists the number of statistically significant variables, $\mathrm{k}_{\text {sig. }}$, at the 90 percent confidence level identified in the hierarchical regressions (HR), backwards eliminations (BE), stepwise regressions (SR) and that are included in the final model (FM).

The variables included in the analysis were selected after considering significant determinants of risk preference from the literature, a series of control variables that could influence the experimental results and the factor loadings identified in the NLPCA.

Table 25 - Summary of Exploratory Analyses

\begin{tabular}{|c|c|c|c|c|c|c|}
\hline \multirow{2}{*}{ Model } & HR & \multicolumn{2}{|c|}{ BE } & \multicolumn{2}{|c|}{ SR } & FM \\
\cline { 2 - 7 } & $\mathrm{k}_{\text {sig. }}$ & $($ Entry, Exit) & $\mathrm{k}_{\text {sig. }}$ & (Entry, Exit) & $\mathrm{k}_{\text {sig. }}$ & $\mathrm{k}_{\text {sig. }}$ \\
\hline $\boldsymbol{\theta}^{+}$ & 7 & $(.3, .5)$ & 16 & $(.4, .5)$ & 8 & 9 \\
\hline $\boldsymbol{\theta}^{-}$ & 7 & $(.2, .3)$ & 22 & $(.3, .5)$ & 7 & 9 \\
\hline $\boldsymbol{\delta}^{+}$ & 8 & $(.1, .2)$ & 12 & $(.3, .5)$ & 8 & 11 \\
\hline $\boldsymbol{\gamma}^{+}$ & 2 & $(.1, .2)$ & 16 & $(.3, .5)$ & 7 & 11 \\
\hline $\boldsymbol{\delta}^{-}$ & 8 & $(.05, .1)$ & 32 & $(.3, .5)$ & 8 & 15 \\
\hline $\boldsymbol{\gamma}^{-}$ & 2 & $(.05, .1)$ & 36 & $(.3, .5)$ & 20 & 23 \\
\hline $\boldsymbol{\lambda}$ & 5 & $(.05, .15)$ & 32 & $(.35, .5)$ & 20 & 15 \\
\hline
\end{tabular}

\subsubsection{Case Removal}

Prior to building the regression models for each of the model parameters, outliers, extreme values and observations exerting undue influence on the model were identified and considered for exclusion. Problematic observations are characterized by standardized values greater than 3.29 or less than -3.29 , a Cook's distance $>1$ and centered leverage values $>3 *(k+1) / \mathrm{n}($ Stevens, 2002). This was done to find dominated responses or otherwise illogical answers. The criteria for exclusion of an outlier, extreme value or observation exerting undue influence on the model was two or more strictly dominated responses in a given section. This is more conservative than the criteria in the initial data cleaning process which excluded participants who provided three or more strictly dominated responses. The number of excluded cases as well as the initial and final sample sizes for each section are presented in Table 26. 
Table 26 - Sample Sizes and Excluded Cases by Section

\begin{tabular}{|l|c|c|c|}
\hline Section & Initial Sample $(\mathrm{n})$ & Excluded Cases & Final Sample $(\mathrm{n})$ \\
\hline Utility in the gain domain & 184 & 10 & 174 \\
\hline Utility in the loss domain & 184 & 13 & 171 \\
\hline Decision weights in the gain domain & 144 & 30 & 114 \\
\hline Decision weights in the loss domain & 123 & 50 & 73 \\
\hline Loss aversion & 144 & 44 & 100 \\
\hline
\end{tabular}

\subsubsection{OLS Regression Models}

Table 27 presents seven separate OLS regressions exploring the effect of visceral factors on each of the parameters that characterize risk preference under CPT. The regression models were derived using the OLS estimation method with robust estimators where applicable. Each of the parameters include nine stable factors, eight visceral factors, and an average of five interaction effects.

Table 27 - OLS Regression Models for each of the Seven Parameters

\begin{tabular}{|c|c|c|c|c|c|c|c|}
\hline & $\begin{array}{c}\boldsymbol{\theta}^{+} \\
(\mathrm{n}=174)\end{array}$ & $\begin{array}{c}\boldsymbol{\theta}^{-} \\
(\mathrm{n}=171)\end{array}$ & $\begin{array}{c}\boldsymbol{\delta}^{+} \\
(\mathrm{n}=114)\end{array}$ & $\begin{array}{c}\gamma^{+} \\
(n=114)\end{array}$ & $\begin{array}{c}\boldsymbol{\delta}^{-} \\
(\mathrm{n}=73)\end{array}$ & $\begin{array}{c}\gamma^{-} \\
(n=73)\end{array}$ & $\begin{array}{c}\lambda \\
(n=85)\end{array}$ \\
\hline \multicolumn{8}{|l|}{ Stable Factors } \\
\hline (Constant) & .483 & $1.034 * *$ & $5.256^{*}$ & .096 & $8.208 * * *$ & $-4.730 * * *$ & -.245 \\
\hline Age & -.006 & -.007 & -.031 & .033 & $-.436 * * *$ & $.092 * * *$ & .056 \\
\hline Gross wealth & $1.2 \mathrm{E}-7$ & $5.9 \mathrm{E}-7$ & $<.001 * *$ & $-7.8 \mathrm{E}-5^{*}$ & $8.6 \mathrm{E}-5^{* *}$ & $<.001^{* * *}$ & $-6.8 \mathrm{E}-6 * * *$ \\
\hline Gender (Male=1) & .043 & -.047 & -.163 & .123 & $-1.052 * *$ & .094 & $.545^{* * *}$ \\
\hline Post-secondary degree & -.099 & -.126 & -.761 & $-1.086^{* *}$ & $4.427 * * *$ & $-.525 *$ & $2.538 * * *$ \\
\hline Part-time & -.024 & -.089 & -.093 & .174 & -.745 & .115 & $-.340 *$ \\
\hline Extraversion & .039 & -.005 & -.102 & -.068 & $-.806 * * *$ & $-.240 * * *$ & .026 \\
\hline Agreeableness & .022 & -.010 & -.259 & .215 & -.119 & $.160 * *$ & .059 \\
\hline Conscientiousness & .032 & .074 & -.036 & .060 & $1.272 * * *$ & $.817^{* * *}$ & $-.220 *$ \\
\hline Neuroticism & $.059 *$ & -.058 & -.540 & .117 & -.170 & $.442 * * *$ & .128 \\
\hline \multicolumn{8}{|l|}{ Visceral Factors } \\
\hline Angry & $-.151 * *$ & $.127^{*}$ & .063 & -.002 & $-.204 *$ & .026 & $-.478 * * *$ \\
\hline Sad & $-.024 *$ & $-.197 * *$ & $-1.340 * *$ & $-.447 * *$ & .017 & $-.210 * * *$ & $.778 * * *$ \\
\hline Happy & -.018 & $.034 * *$ & $.145 *$ & $-.151 * * *$ & $1.380 * * *$ & $.338 * * *$ & $.211 * * *$ \\
\hline Anxious & -.017 & .096 & $.775^{* *}$ & $.547^{*}$ & $-.968 * *$ & .017 & $-.146 * *$ \\
\hline Hungry & -.011 & .013 & -.057 & $.178^{* *}$ & .112 & $.221 * * *$ & -.051 \\
\hline Energetic & $.044 * * *$ & .009 & $-.176 * *$ & .123 & -.050 & $-.134 * * *$ & $-.161 * * *$ \\
\hline Tired & $.025 * * *$ & $-.023 *$ & -.061 & -.026 & .146 & $-.104 * * *$ & $-.075^{*}$ \\
\hline Stressed & .008 & .006 & .058 & -.068 & -.165 & $.195 * * *$ & -.012 \\
\hline \multicolumn{8}{|l|}{ Interaction Terms } \\
\hline Angry*Stress & $.009 * *$ & $-.014 *$ & & & & & \\
\hline \multicolumn{8}{|l|}{ Angry*Hunger } \\
\hline Angry* Energetic & $.016^{* * *}$ & & & & & $.021 * *$ & \\
\hline Angry*Anxious & & & & & & & $.098^{* * *}$ \\
\hline Sad*Tired & & $.016 * * *$ & & & & $.056 * * *$ & $-.049 * * *$ \\
\hline Sad*Stressed & & & $.062^{* *}$ & & & $-.064 * * *$ & \\
\hline Happy*Hungry & & $-.007 * *$ & & & & & \\
\hline Anxious*Hungry & & & & -.016 & & $-.067 * * *$ & $.039 * * *$ \\
\hline Anxious*Stressed & & & $-.058 * *$ & & & $.032 * * *$ & \\
\hline Hungry*Energetic & & & & -.017 & & & \\
\hline
\end{tabular}




\begin{tabular}{|c|c|c|c|c|c|c|c|}
\hline Energetic*Stressed & $-.007 * *$ & & & & & & \\
\hline Consc.*Sad & & & & $.143 * *$ & & & \\
\hline Consc. *Anxious & & $-.030 *$ & & $-.093^{*}$ & & & \\
\hline Neuroticism*Sad & & .028 & $.298^{*}$ & & & & $-.116 * * *$ \\
\hline Neuroticism*Anxious & & & -.115 & -.059 & $.354^{* * *}$ & & \\
\hline \multicolumn{8}{|l|}{ Neuroticism*Stressed } \\
\hline Neuroticism*Happy & & & & & $-.321 * * *$ & $-.086 * * *$ & \\
\hline Neuroticism*Angry & $.027 * *$ & & & & & & \\
\hline Gross wealth*Consc. & & & $-3.4 \mathrm{E}-5^{*}$ & $1.6 \mathrm{E}-5^{*}$ & & $-8.6 \mathrm{E}-5 * * *$ & \\
\hline Gross wealth*Anxious & & & $-1.9 \mathrm{E}-5^{* * *}$ & $8.4 \mathrm{E}-6 * *$ & & $3.0 \mathrm{E}-5^{* * *}$ & \\
\hline Gross wealth*Tired & & & & & $-2.1 \mathrm{E}-5^{* *}$ & & \\
\hline Gross wealth* Stressed & & & & & & $-3.1 \mathrm{E}-5 * * *$ & \\
\hline \multicolumn{8}{|l|}{ Model Diagnostics } \\
\hline $\mathrm{R}^{2}$ & .367 & .334 & .394 & .358 & .713 & .848 & .800 \\
\hline Adj. $R^{2}$ & .232 & .182 & .154 & .088 & .544 & .707 & .692 \\
\hline Durbin-Watson & 2.078 & 2.019 & 1.723 & 1.974 & 1.654 & 2.627 & 1.993 \\
\hline Breusch-Pagan (sig.) & .664 & .024 & .001 & .135 & .973 & .936 & .234 \\
\hline Koenker test (sig.) & .438 & .287 & .383 & .907 & .682 & .394 & .042 \\
\hline Kolmogorov-Smirnov (sig.) & .200 & .085 & .057 & .050 & .200 & .200 & .200 \\
\hline Shapiro-Wilk (sig.) & .922 & .187 & $<.001$ & .001 & .538 & .551 & .918 \\
\hline
\end{tabular}

$*$ The significance tests of the parameters coefficients in the models that violate multivariate normality are based on standard errors calculated using bias-corrected and accelerated (BCa) bootstrap intervals with 1000 bootstrap samples.

** The significance tests of the parameter coefficients in models that violate the homoscedasticity assumption are based on $\mathrm{HC} 4$ variant heteroscedasticity-consistent standard errors.

Figure 13 illustrates graphically the magnitude of visceral factor effects. The vertical axis of each graph represents the size of the average effect as a percentage of the mean parameter estimate. Whether the effect corresponds to increased risk-seeking or risk-aversive behaviour is illustrated by a light red and blue gradient, respectively.

Figure 13 - Average Magnitude of Significant Visceral Factor Effects

a) Loss Aversion
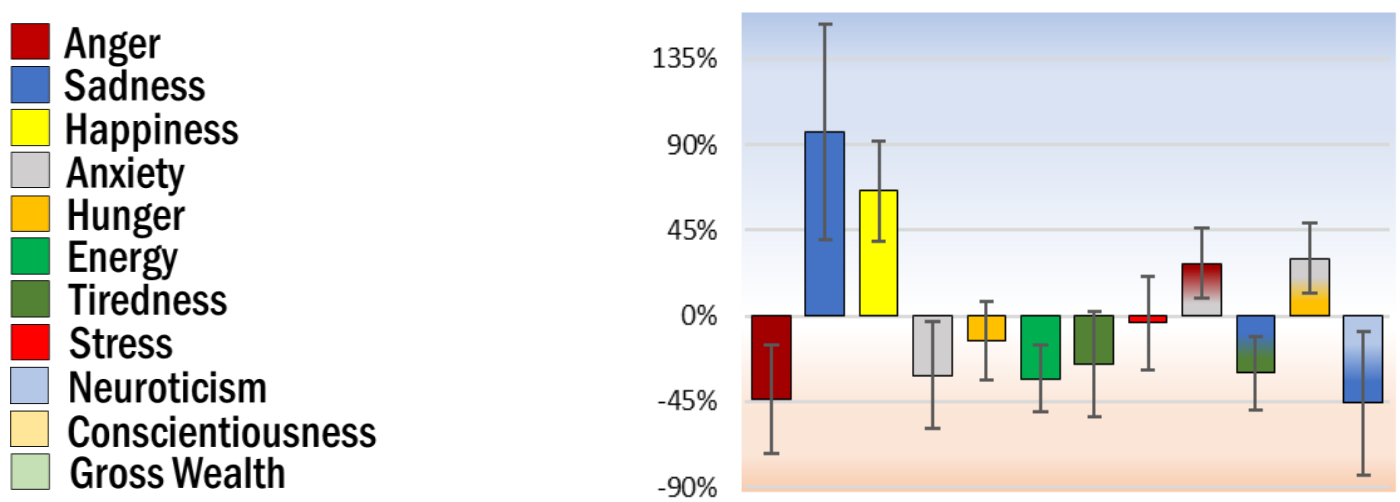
b) Utility in the Gain Domain

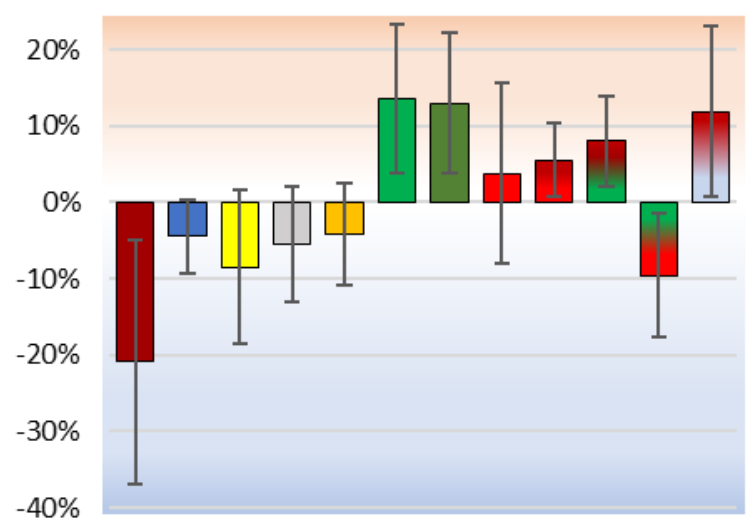

d) Elevation in the Gain Domain

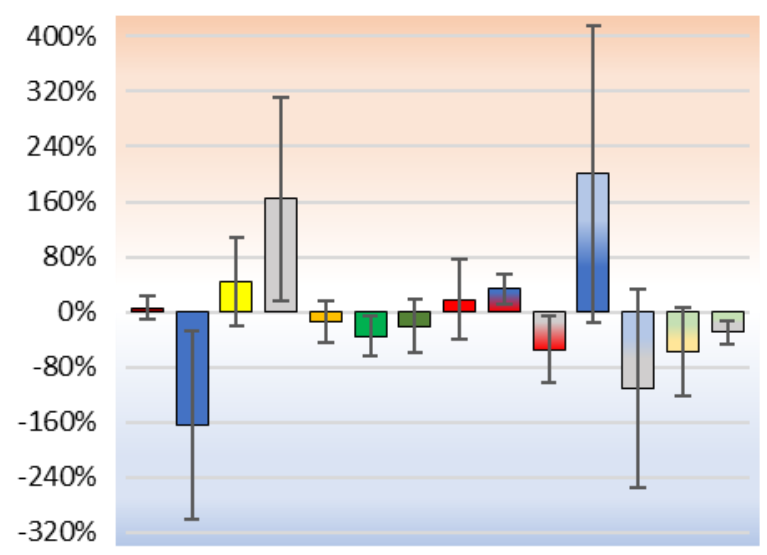

f) Elevation in the Loss Domain

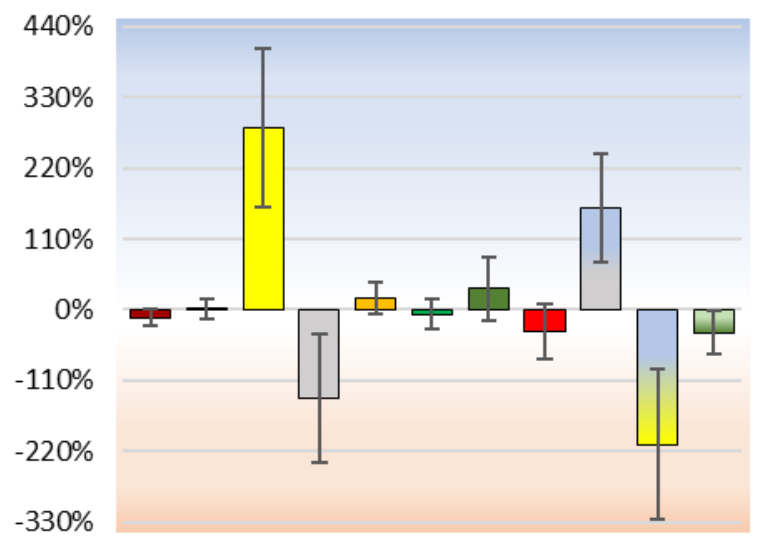

c) Utility in the Loss Domain

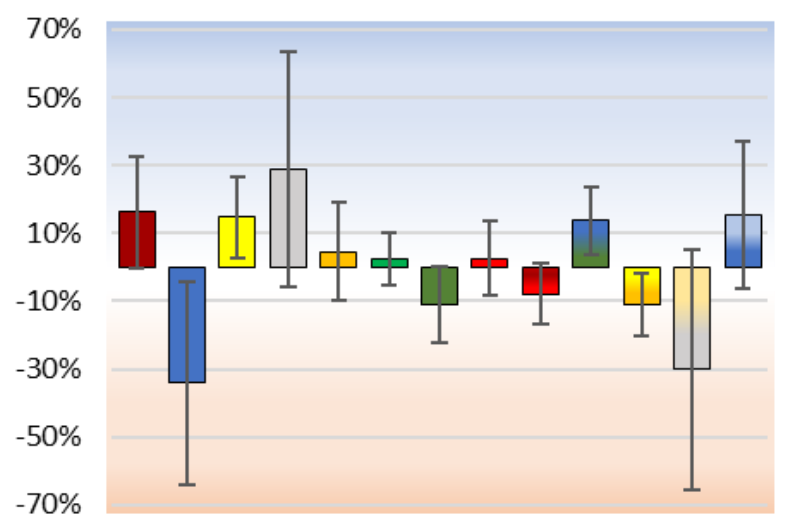

e) Curvature in the Gain Domain

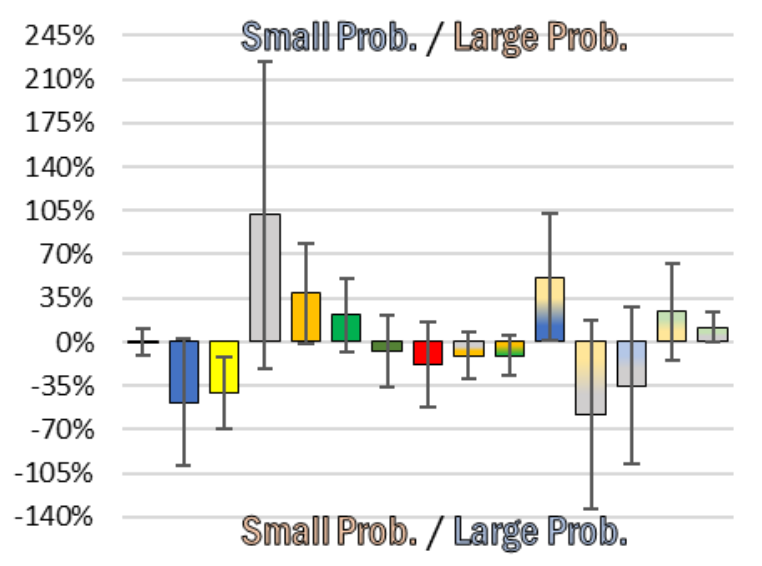

g) Curvature in the Loss Domain

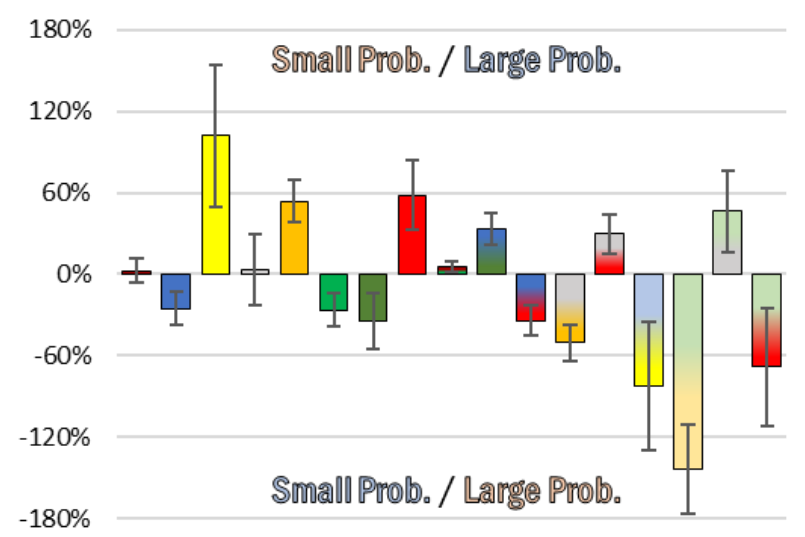




\subsubsection{Visceral Factor Effects}

Anger and related interaction effects are found to have the most significant effect on the shape of the utility function in the gain domain, $\theta^{+}$, and the magnitude of the loss aversion parameter, $\lambda$. Consistent with findings from Campos-Vazquez \& Cuilty (2014), this study finds anger significantly related to a decrease in loss aversion, $\lambda$. This implies an increase in risk-seeking behaviour in mixed gambles and acts to significantly moderate an increase in risk aversion implied by changes in the shape of the utility function. Contrary to findings presented by Conte, Levati \& Nardi (2016) and my initial hypothesis, this study finds anger related to increased aversion to risk in gain and loss gambles through increased concavity and decreased convexity of the utility function in the gain and loss domains, respectfully. This effect is moderated in the gain domain in participants exhibiting high levels of neuroticism and energy, and by participants experiencing high levels of stress in both domains.

This study finds sadness mildly related to increased risk aversion in the gain domain and strongly related to increased risk seeking in the loss domain which is consistent with evidence presented by Treffers, Koellinger \& Picot (2016) and Campos-Vazquez \& Cuilty (2014), but contrary to my initial hypotheses. Sadness is found to have a significant effect on all the parameters of interest with the exception of the elevation decision weight parameter in the loss domain, $\delta^{-}$. The increased aversion to risk in the gain domain is a product of mild decreases in the power parameter, $\theta^{+}$, and strong decreases in the elevation of the decision weight parameter in the gain domain, $\delta^{+}$. Increased risk seeking in the loss domain is the result of strong decreases in the power parameter which corresponds to an increase in the convexity in the utility function. This effect is moderated by strong increases in the loss aversion parameter, $\lambda$, as well as in participants exhibiting high levels of neuroticism and tiredness. Sadness is also found to be related to decreases in the curvature decision weight parameter, $\gamma$, in both the gain and loss domains. This implies increased sensitivity to changes in probabilities close to zero and one as well as an increase in the overweighing of probabilities close to zero and under-weighing of probabilities close to one. In the gain domain, this effect is moderated through an interaction between sadness and conscientiousness. In the loss domain, the effect of sadness is moderated through an interaction with anxiousness and exacerbated through an interaction with stress. 
There is no statistically significant relationship found between happiness and risk preference in the gain domain which is consistent with the findings presented in Callen et al. (2014) and Treffers, Koellinger \& Picot (2016) and contrary to reductions in aversion to risk found by Conte, Levati \& Nardi (2016). Happiness is, however, found to decrease risk-seeking behaviour in the loss domain through a mild positive relationship with the power parameter, $\theta^{-}$, and a strong positive relationship with the elevation decision weight parameter, $\delta^{-}$. The effect of happiness on the power parameter causes an increase in the convexity of the utility function which is moderated by its interaction with hunger. The effect on the elevation parameter is more pronounced but moderated in participants exhibiting higher levels neuroticism. Happiness is also found to have opposing effects on the curvature decision weight parameter, $\gamma$; decreasing the parameter in the gain and increasing it in the loss domain. This implies an increase and decrease in the sensitivity of participants to changes in probabilities close to zero and one in the gain and loss domains, respectfully. Lastly, this study also reveals a moderate positive relationship between happiness and the loss aversion parameter indicating that participants exhibiting higher levels of happiness were more risk averse in mixed gambles.

My findings indicate that anxiety is related to increased risk-seeking behaviour in both the gain and loss domains, though it is not found to have a significant effect on the curvature of the utility function. As hypothesized, the coefficient for anxiety is negative in the gain domain and positive in the loss domain, which implies greater aversion to risk, though statistical tests do not reveal a significant effect on the power parameter in either domain. Anxiety is, however, found to significantly reduce loss aversion which implies a greater willingness to accept risk in mixed gambles. Anxiety is also found to be significantly related to increased probabilistic risk seeking through positive and negative relationships with elevation decision weight parameter, $\delta$, in the gain and loss domains, respectively. This effect is moderated by its interaction with stress and gross wealth in the gain domain and in participants exhibiting higher levels of neuroticism in both domains. Lastly, anxiety is found to significantly affect the curvature decision weight parameter in the loss domain, $\gamma^{-}$, though its interaction with hunger, stress and gross wealth.

Contrary to findings presented by Symmonds et al. (2010), this study finds no significant effect of hunger on risk preference in isolation from interaction effects with other visceral factors. There are, however, previously stated interaction effects between hunger and happiness 
on the power parameter in loss domains that moderates the relationship between these factors and risk aversion in both domains. This implies that hunger may result in risk-seeking behaviour, but only through its interaction with other visceral factors. There is also a mild negative relationship found between hunger and loss aversion which could lead to increased risk-seeking behaviour, though this effect is not statistically significant and strongly mitigated in the presence of anxiousness. This study does find there to be a significant positive effect of hunger on the curvature decision weight parameter in both the gain and loss domains which is moderated in the loss domain through an interaction between hunger and anxiety. This implies decreased sensitivity to changes in probabilities close to zero and one as well as a decrease in the overweighing of probabilities close to zero and under-weighing of probabilities close to one.

Sleep deprivation is measured using two unipolar visual analogue scales; one labelled 'energetic' and the other 'tired'. Although I do find there to be a negative correlation between the two factors, this study does not find opposing effects on risk preference as hypothesized. Instead I find both factors to be positively related to risk-seeking behaviour through a positive relationship with the power parameter in the gain domain, $\theta^{+}$, and a negative relationship with the loss aversion parameter, $\lambda$. The effect of energy on the power parameter is moderated by its interaction with stress and exacerbated by its interaction with anger while the effect of tiredness on the loss aversion parameter is exacerbated by its interaction with sadness.

Both energy and tiredness are also related to decreases in the curvature decision weight parameter in the loss domain and are again found to interact significantly with anger and sadness, respectively. The interaction effects, in this case, have a moderating the effect of the visceral factors on the curvature parameter contrary to the exacerbating effect that they have on the power and loss aversion parameters. This implies that participants exhibiting high levels of energy and anger and/or high levels of tiredness and sadness are less prone to risk-seeking behaviour when there is a large probability of obtaining the worst-case scenario in the loss domain than they would be had the interacting visceral factors not been present.

A moderate relationship between tiredness and the power parameter in the loss domain is also found, which implies increased risk aversion. When combined with the positive relationship found with the power parameter in the gain domain, these findings are consistent with those presented by McKenna, Dickinson, Orff, \& Drummond (2007) who found a sleep deprived 
group significantly less risk averse in gains and less risk seeking in losses. This effect is, however, drastically moderated by its interaction with sadness. Lastly, energy is found to have a negative relationship with the elevation decision weight parameter in the gain domain which implies decreased probabilistic risk seeking and acts to moderate the increase in risk-seeking behaviour implied by increases to the power parameter in the gain domain and decrease in the loss aversion parameter. The similar effects of energy and tiredness on risk preference highlights the need to treat theses variables separately and not measure them using a bipolar VAS.

Stress is not found to significantly affect risk preference, though I do find a positive effect on the curvature decision weight parameter in the loss domain which is moderated through its interaction with sadness and with gross wealth and exacerbated through its interaction with anxiousness. Stress in these experiments was most significant on risk preference through its interaction with other visceral factors described in the sections above. When interacting with anger and sadness, stress appears to have a positive relationship with increased risk-seeking behaviour and when interacting with anxiousness and energy we see the opposite effect. The greatest effect of stress on risk preference is found in its effect on the curvature parameter in the loss domain. There is an overall positive effect on less wealthy individuals indicating a decrease in the overweighing of probabilities close to zero and under-weighing of probabilities close to one. This implies greater risk-seeking behaviour in lotteries with a small probability of obtaining the worst-cast scenario and greater risk aversion in lotteries with a high probability of obtaining the worst-case scenario.

\subsection{Hierarchical Regressions}

A series of nested F-tests are performed on the OLS regression models presented above to identify groups of regressors that are statistically significant predictors of each of the parameters of interest. The model variables are organized into three blocks: a block of stable factors, a block of visceral factors, and a block of factor interactions. Model 1 includes the five demographic factors and four personality traits as stable factors, Model 2 adds the eight visceral factors being investigated, and Model 3 adds the interaction terms for each model. Table 28 lists $\mathrm{R}^{2}$ and adjusted $\mathrm{R}^{2}$ values, the change in the $\mathrm{R}^{2}$, the F-statistic, and the significance of the change in the F-statistic for each of the three models. 
Table 28 - Hierarchical (Blockwise Entry) Regression Summary

\begin{tabular}{|l|l|l|l|l|l|}
\hline Model & $\mathrm{R}^{2}$ & Adj. $\mathrm{R}^{2}$ & $\mathrm{R}^{2}$ Change & F Change & Sig. F Change \\
\hline $1\left(\theta^{+}\right)$ & .097 & .024 & .097 & 1.329 & .230 \\
\hline $2\left(\theta^{+}\right)$ & .267 & .146 & .170 & 2.988 & .005 \\
\hline $3\left(\theta^{+}\right)$ & .367 & .232 & .099 & 3.875 & .006 \\
\hline $1\left(\theta^{-}\right)$ & .036 & -.044 & .036 & .448 & .906 \\
\hline $2\left(\theta^{-}\right)$ & .103 & -.048 & .067 & .948 & .481 \\
\hline $3\left(\theta^{-}\right)$ & .334 & .182 & .231 & 6.674 & $<.001^{* * *}$ \\
\hline $1\left(\delta^{+}\right)$ & .092 & -.022 & .092 & .807 & .611 \\
\hline $2\left(\delta^{+}\right)$ & .163 & -.059 & .071 & .681 & .706 \\
\hline $3\left(\delta^{+}\right)$ & .394 & .154 & .231 & 3.692 & .004 \\
\hline $1\left(\gamma^{+}\right)$ & .119 & .009 & .119 & 1.083 & .386 \\
\hline $2\left(\gamma^{+}\right)$ & .195 & -.019 & .075 & .750 & .648 \\
\hline $3\left(\gamma^{+}\right)$ & .358 & .088 & .163 & 2.074 & .061 \\
\hline $1\left(\delta^{-}\right)$ & .249 & .099 & .249 & 1.661 & .127 \\
\hline $2\left(\delta^{-}\right)$ & .466 & .221 & .217 & 1.881 & .093 \\
\hline $3\left(\delta^{-}\right)$ & .713 & .544 & .246 & 9.725 & $<.001^{* * *}$ \\
\hline $1\left(\gamma^{-}\right)$ & .162 & -.006 & .162 & .964 & .482 \\
\hline $2\left(\gamma^{-}\right)$ & .219 & -.140 & .057 & .340 & .945 \\
\hline $3\left(\gamma^{-}\right)$ & .848 & .707 & .629 & 12.881 & $<.001^{* * *}$ \\
\hline $1(\lambda)$ & .453 & .356 & .453 & 4.690 & $<.001^{* * *}$ \\
\hline $2(\lambda)$ & .664 & .532 & .212 & 3.390 & .004 \\
\hline $3(\lambda)$ & .800 & .692 & .135 & 6.599 & $<.001^{* * *}$ \\
\hline & & & & & \\
\hline
\end{tabular}

The results of this analysis indicate that the block of stable factors is a statistically significant determinant of the loss aversion parameter, the visceral factor block is statistically significant on the loss aversion parameter and power parameter in the loss domain, and factor interactions block is statistically significant on all but the curvature decision weight parameter in the gain domain which showed weak significance. These tests illustrate not only the significance of visceral factors relative to stable factors, but also the importance of including visceral factor interactions.

\subsection{Reliability of Stable Determinants as Classifiers}

The panel dataset is used to determine the suitability of the nine stable determinants of risk preference as entity-specific classifiers by testing the OLS regression models presented in Table 27 for significant differences in individual-specific intercepts. This is done by conducting two Breusch-Pagan Lagrange multiplier tests on each of the models: one with and one without the nine stable factors. The first test revealed that there are significant differences across groups while in the second I fail to reject the null hypothesis there are no significant differences for each of the models. Since the first test indicates that the effect of the visceral factors on the parameters of interest are not uniform and that it is necessary to include entity-specific intercepts and the 
second does not, it is determined that the individual-specific intercepts are adequately accounted for in the stable factors selected for each parameter.

Table 29 - Pooled OLS vs. Random-Effects

\begin{tabular}{|l|c|c|c|c|c|c|c|}
\hline & $\begin{array}{c}\boldsymbol{\theta}^{+} \\
(\mathrm{n}=46)\end{array}$ & $\begin{array}{c}\boldsymbol{\theta}^{-} \\
(\mathrm{n}=41)\end{array}$ & $\begin{array}{c}\boldsymbol{\delta}^{+} \\
(\mathrm{n}=39)\end{array}$ & $\begin{array}{c}\boldsymbol{\gamma}^{+} \\
(\mathrm{n}=38)\end{array}$ & $\begin{array}{c}\boldsymbol{\delta}^{-} \\
(\mathrm{n}=27)\end{array}$ & $\begin{array}{c}\boldsymbol{\gamma}^{-} \\
(\mathrm{n}=23)\end{array}$ & $\boldsymbol{\lambda}(\mathrm{n}=43)$ \\
\hline Breusch-Pagan LM \#1: & 4.61 & 3.92 & 6.69 & 8.04 & 3.02 & 5.79 & $<.001$ \\
chibar(01) (Prob > chibar) & $(.0159)$ & $(.0239)$ & $(.0049)$ & $(.0023)$ & $(.0410)$ & $(.008)$ & $(1.00)$ \\
\hline Breusch-Pagan LM \#2: & .23 & 0.00 & .03 & 2.17 & 0.00 & 0.00 & 0.00 \\
chibar(01) (Prob > chibar) & $(.3150)$ & $(1.00)$ & $(.4505)$ & $(.0705)$ & $(1.00)$ & $(1.00)$ & $(1.00)$ \\
\hline
\end{tabular}




\section{CHAPTER 7 \\ DISCUSSION, APPLICATIONS AND CONCLUSION}

\subsection{Discussion}

It is common for people to believe they are willfully in control of their decisions and that there is a direct causal link between their own thoughts and actions (Wegner \& Wheatley, 1999). As a result, they tend to exaggerate the effect of higher-order cognitive processes and downplay the role of unconscious influencers like visceral factors when reflecting on past decisions (Loewenstein, 2000). Visceral factors play a central role in decision making, though people consistently underestimate their effect on their own future behaviour and easily forget past experiences of their behavioural implications (Loewenstein, 1996). This suggests that not only are decision makers likely unaware that their decisions are being influenced by visceral states, but also that the effects of these states are pervasive and enduring.

I can safely conclude that visceral factors affect decision making by altering the disposition people have towards risk. This study provides evidence to show that the effect of visceral states on risk preference is the result of their combined effects on the way people subjectively value outcomes and weigh probabilities. The parameter estimates listed in Table 27 provide a reasonably accurate depiction of the effect of visceral factors on risk preference.

\subsubsection{The Significance of Interaction Effects}

Unlike stable factors and cognitive limitations affecting risk preference, the body of knowledge on the effect of emotions and other visceral factors is relatively underdeveloped. Many of the studies contributing to our understanding of these factors explore their effects independently of one another and rarely do they account for interactions between visceral factors. Of the reviewed studies that adopt a distinct-states approach to measuring the effect of emotions on risk preference, none were found to explore the effect of the states alongside other visceral factors or interactions between different states occurring simultaneously. By omitting interaction effects, the resulting models fail to capture an important dynamic amongst visceral factors and risk preference.

My findings indicate that interaction effects are significant determinants of risk preference. Through a series of nested F-tests presented in Table 28, I identify the block of visceral factor 
interactions as statistically significant in all but one of the seven model parameters. This is compared to the block of stable factors which is significant in one parameter and the block of visceral factors which is significant in two parameters.

The significance of these tests provides evidence of the interconnectedness of visceral factors, some of which are found to be significant only through their interaction with other visceral factors. Hunger and stress, for example, directly affect risk preference only through their effect on the sensitivity of the participants to changes probabilities. In other words, neither hunger nor stress are directly related to an unambiguous change in either risk-seeking or risk-aversive behaviour independent of the context of the gambles used to elicit risk preference. The results of this study suggest that both factors may lead to increased risk-seeking behaviour, but only through their interaction with other visceral factors. This presents a potential conceptual difficulty in experiments exploring the effect of hunger or stress on risk preference in isolation from other potentially strongly correlated visceral factors. By not controlling for other visceral factors and their interactions researchers may be inflating the significance of these factors on risk preference and overlooking an important dynamic that is instrumental in the formation of risk preference.

\subsubsection{Overgeneralization of Visceral Factors and Risk Preference}

The overgeneralization of visceral factors and risk preference is a relatively common and problematic practice in the reviewed literature. Many of the earlier studies exploring the effect of visceral states on risk preference adopted a dimensional approach which resulted in largely contradictory findings (Conte et al. 2016). Similarly, risk preference is commonly defined too broadly which overlooks effects on the elements that make people more or less risk averse. This study not only finds that visceral factors should be treated as distinct states but also that risk preference should be decomposed into its constituent components.

One method often used to describe non-reflective feelings measures core affect along three continuums: pleasure \& displeasure, tension \& relaxation, and energy \& tiredness (Ekkekakis, 2012). While this measure is highly referenced and often utilized, this study finds evidence which raises concerns about characterizing visceral states using bipolar scales. The direct effects of energy and tiredness on risk preference, for example, are found to be largely confined to realm of utilities and, surprisingly, the effects are similar. I find both factors directly relate to risk- 
seeking behaviour through decreased aversion to losses and increased risk seeking in the gain domain.

This study also finds that the effect of two unpleasant affective states, anger and anxiety, also differ in their effect on risk preference. While anger relates to increased aversion to risk, anxiety is found to increase probabilistic risk-seeking behaviour by making participants more optimistic about favourable outcomes. This finding provides evidence to support concerns raised in previous studies about the inadequacy of valence-based approaches in capturing conflicting effects of distinct states of a similar valence (Kugler, Connolly \& Ordóñez, 2012; Lerner \& Keltner, 2001; Lerner et al. 2015).

Similar to how emotions of the same valence have been found to have conflicting effects on risk preference (Smith \& Ellsworth, 1985; Lerner \& Keltner, 2000), my findings indicate that distinct visceral states also affect different elements of risk preference. Evidence presented in this study suggests that the common generalization of risk preference as either more or less risk averse may be confounding the largely inconclusive results presented in the reviewed literature. Anger, for example, is found to only affect risk preference through the valuation of outcomes while the effects of anxiety are confined exclusively to the weighting of probabilities. This finding indicates not only that these emotions should be treated as distinct states but also that the common generalization of risk preference is too broad to be descriptively meaningful.

\subsection{Applications}

Decision sciences are critical to the development of our understanding of the nature of decision making and the mechanisms that guide human behaviour and social interaction. The decision theory used in this study is adept at capturing the more pervasive cognitive limitations but can

result in poor fit and systematic violations when tested empirically (Birnbaum, 2005; Birnbaum, Patton \& Lott, 1999). According to Loewenstein (1996), a central barrier in applying decision theories to real-world applications is their failure to account for the effects of visceral factors on decision making. By outlining the effects of visceral factors on the elements that define risk preference under CPT, my findings can be used to improve the practical applicability of CPT in areas such as finance, public relations and an emerging industry known as affective computing. 


\subsubsection{Finance}

Market prices often deviate from their intrinsic value for reasons that can't be explained under many classic valuation theories, but what is less known is how and why this occurs. I have show that visceral factors play a significant role in shaping the way we subjectively value outcomes and weigh probabilities; both of which have important implications on stock misvaluation.

Classic economic valuation theories value companies based on their intrinsic value and cannot account for stock prices that deviate substantially from their intrinsic value for extended periods

of time (Sornette, 2000; Kumar \& Lee, 2006; DellaVigna, 2009). This is, in part, because they do not account for cognitive biases, visceral states and other irrational determinants of retail investment behaviour. Systematic stock misvaluation has been linked to loss aversion (Shefrin \& Statman, 1985), optimism and pessimism (Jouini \& Napp, 2010), investor overreaction (De Bondt \& Thaler, 1984; 1987), overall market sentiment (Kumar \& Lee, 2006; Chang, Luo \& Ren, 2013), distinct emotional states induced by blog posts (Xu et al. 2017) as well as upbeat moods in what is termed 'the sunny day effect' (Saunders, 1993; Hirshleifer \& Shumway, 2003). Emotions, in particular, play a central role in shaping market prices and are thought to be the most important factor causing bull markets (Shiller, 2000).

One of the more direct implications of my findings in finance is to inform investors of how their judgement may be affected by visceral factors. Since these factors act on an unconscious level, many investors are likely unaware that their preferences change depending on their current visceral state (Critchley \& Harrison, 2013). People also tend to underestimate the effect of visceral factors on their own actions (Loewenstein, 1996; Wegner \& Wheatley, 1999) which increases the likelihood that they are not heavily considered when making decisions. In fastpaced industries such as finance, it is important for traders and wealth management specialists to know when and how their judgement is being influenced. If, for example, an investor is aware that when they are anxious, they tend to underweight the probability of obtaining the worst-case scenario, they are able to adjust for this bias when it is prudent to make conservative decisions.

With so much of the market driven by cognitive biases and emotion, it is essential that companies take these factors into consideration when communicating information to investors. There are countless examples of company announcements or reports that have caused significant swings in stock prices. On July 26, 2018, for example, the stock price of Facebook, Inc. dropped 
roughly 20 percent, worth approximately $\$ 120$ billion in USD, after releasing a weak guidance report and an earnings report that failed to meet expectations. Large and sudden changes in stock prices such as this highlight the importance of anticipating the emotions that company announcements will generate. The overreaction of investors to new information and extreme events are believed to be the result of unjustified optimism or pessimism, fear, greed and surprise (De Bondt, 1989; Piccoli \& Chaudhury, 2017). With an understanding of the effect of emotions on the way people interpret information, public relation specialists can control anticipated investor responses to company announcements or unexpected events by crafting messages in a way that will moderate adverse effects on the market price of the stock.

As a final note on the applications of my findings in finance, this thesis also provides important information for financial advisors in knowing their clients. In many countries, financial advisors are required to develop investment strategies consistent with their client's risk preference. Since risk preferences depend on visceral states, the issue arises whether financial advisors should measure and account for these factors before making portfolio recommendations.

According to a study conducted by PlanPlus Inc. for the Ontario Securities Commission (OSC), the questions that financial advisors use to elicit individual preferences do not give an accurate representation of the client's risk preference and are not suitable for constructing risk profiles (Brayman et al. 2015; Isrealson, 2016; Noakes, 2016). Advisory firms are responsible for developing questionnaires consistent with the OSC's Know-Your-Client rule, but few advisors have the training necessary to construct such tools. In some cases, these questionnaires have even been found to elicit profiles that favour more profitable products for the advisors (Noakes, 2016). In fact, $85 \%$ of wealth management firms in Canada were found to have inadequate questionnaires which means that advisors simply do not have the information necessary to know what is best for their clients (Brayman et al. 2015). Often these questionnaires consist of only a few ambiguously worded questions and are rarely taken seriously by advisors (Palma \& Picard, 2010). The unfortunate result is an inadequate risk profile which leads to a discrepancy between what the profile determines to be in the client's best interest and what is actually best for the client.

Not surprisingly, the most common complaint received by regulators like the OSC has, for many years, been that a client's money was invested in positions that did not make sense for 
them (Noakes, 2016; Brayman et al. 2015; OBSI, 2014). These and other complaints can often be traced back to poor risk profiling which is one of the leading theories behind recent breakdowns in client-advisor relationships (Noakes, 2016). By constructing more comprehensive risk preference profiles financial advisors can better advise their clients which will in turn foster more trust between clients, their advisors and wealth management firms in general.

\subsubsection{Machine-Human Interaction}

As machine-human interaction becomes more prevalent in society there is an increasing demand for machines capable of understanding human emotion and interacting more effectively with humans. When paired with emotion analytic technology, the applications for systems capable of detecting visceral states and applying state-specific decision theories to better anticipate human actions have far-reaching implications. These systems can be applied to enhance driver assistance, safety \& collision avoidance systems in vehicles (Cai \& Lin, 2007; Eyben et al. 2010; Agrawal, Giripunje \& Bajaj, 2013), create socially intelligent virtual assistants (Ball \& Breese, 2000; Fong, Nourbakhsh \& Dautenhahn, 2003) or design video games to trigger real-time changes in gameplay (Liu et al. 2009; Hudlicka, 2009).

The erratic and transitory nature of many visceral states makes the development of statespecific decision theories difficult and, until recent advancements in affective computing, largely impractical. Recent technological advances have made not only the construction of more comprehensive theories pragmatic but have also made possible an important advancement to the field of affective computing.

A lot of the recent progress in affective computing and emotion analytics involve developing a superhuman ability to identify emotions in real-time through visual or physiological cues. The occurrence of these emotions is then assessed by humans to better understand themselves, others or to make changes to various initiatives. The next stage in this arena is to develop a computational understanding of how those emotions elicit actions or responses enabling the technology to interpret these states as a predisposition to think or behave in a certain way. The inclusion of visceral states in decision theories will provide a key ingredient in facilitating this next stage in the progression of affective computing. With this, we will be one step closer to teaching computers how to interact more effectively with humans as well as provide them with a 
level of social intelligence that will better enable them to develop strategies and make judgements in situations involving humans.

\subsection{Conclusion}

This study attempts to identify which visceral factors significantly influence risk preference and to understand the mechanisms underlying this relationship. Using a sample collected from students and faculty at Ryerson University, I elicited individual-level utility and probability weighting functions in both the gain and loss domains as well as an index for loss aversion. By decomposing risk preference into three independent elements elicited using non-parametric methods and measuring the effects of eight visceral factors on the subjective interpretation of gamble attributes, I am able to explain why visceral factors affect risk preference.

The results of the non-parametric utility and decision weight analyses confirm the suitability of the power parameter and the two-parameter probability weighting function (Gonzalez \& $\mathrm{Wu}$, 1999) in capturing the shape of the utility and probability weighting functions. The nonparametric utility analysis also helps to confirm that the majority of individual-level utility functions are convex (risk seeking) in losses and concave (risk averse) in gains.

The decision weight analyses do not find evidence to support the most commonly reported inverse S-shaped probability weighting function. Instead the median decision weight values and elicited decision weight parameters correspond to an S-shaped weighting function in both gains and losses. A series of two-tailed paired samples t-tests indicate that the probability weighting function crosses linearity at points that are reasonably consistent with median estimates in the reviewed literature. These points imply that participants are pessimistic over a greater range of probabilities in the loss domain and therefore exhibit greater probabilistic risk aversion in losses.

An analysis of the panel data reveals considerable heterogeneity across participants. The results of two Breusch-Pagan LM tests on a random-effects model, one with and one without the inclusion of stable determinants of risk preference, indicate that there are significant differences across participants that are effectively accounted for with the inclusion of nine stable factors as regressors: age, gross wealth, gender, post-secondary degree, working part-time, as well as measures of extraversion, agreeableness, conscientiousness and neuroticism. The presence of panel effects in the first test implies a need to allow the intercepts to vary by entity, whereas 
absence of panel effects when the stable factors are included implies that the set of stable factors provide enough differences in individual-level parameter estimates to account for the heterogeneity across participants in the sample. Furthermore, the reliability analysis finds only one significant difference in parameter estimates between trials indicating that there are no systematic effects in the testing procedure that might bias the results.

My results demonstrate that individual-level risk preference is directly related to the type and intensity of eight visceral factors experienced by participants: anger, sadness, happiness, anxiety, hunger, energy, tiredness and stress. Of these, sadness is found to have the most significant effect on risk preference followed by anxiety, happiness and anger while hunger and stress are significant only in their interaction with other visceral factors. The effect of sadness on the model parameters implies that it relates to decreased risk aversion in the gain domain and risk seeking in the loss domain. In most cases, this acts to exacerbate participants' predisposition to risk; making participants who are already risk averse in gains and risk seeking in losses, more risk averse in the gain domain and more risk seeking in the loss domain.

Happiness and anger are both related to increased aversion to risk while anxiety has the opposite effect. I find that happiness acts to increase aversion to risk through changes to the value and weight placed on outcomes and probabilities in the loss domain as well as through an increase in loss aversion. Anger increases aversion to risk in both the gain and loss domains which is moderated by decreases in loss aversion. Anxiety is found to increase risk-seeking behaviour through decreases in loss aversion, the overweighting of the probability of obtaining the best-case scenario in gain gambles and the underweighting of the probability of obtaining the worst-case scenario in loss gambles. Anger and anxiety not only have opposing effects on risk preference, but also differ in the mechanisms through which this occurs. The effect of anger on risk preference is confined exclusively to the valuing of outcomes while anxiety affects only the weighting placed on probabilities. Lastly, the significance of interaction effects among visceral factors highlights the importance of assessing the impact of multiple factors simultaneously.

My findings support the characterization of visceral factors as distinct states and the decomposition of risk preference into its constituent components. The variability in the magnitude and direction of each visceral factor on the distinct elements that define risk preference under CPT indicates that the generalized approaches to characterizing visceral factors 
and risk preference are too broad to be descriptively meaningful when exploring the effect of visceral factors on risk preference.

The effects of visceral factors on the elements that define risk preference under CPT provide insight into not only the potential impact of these factors on decision making but also the sensitivity of risk preference to transitory and erratic factors. While the results of this study are promising, more research is needed understand the systems underlying the relationship between visceral factors and decision making.

One promising area of research explores the effect of visceral factors on decision-making under risk by linking the effects of these factors to the brain systems responsible for the subjective valuation and interpretation of information. The central tenet of this approach suggests visceral states trigger actions or behaviours by disturbing the neurological systems in a way that alters the anticipated satisfaction of a choice or action. In developing a deeper understanding of how visceral states act to intensify or suppress the activation of brain regions responsible for decision-making we can begin to map the effects of visceral states on choice preferences and develop theories grounded in traditional scientific methods. 


\section{CHAPTER 8 \\ LIMITATIONS AND FUTURE WORK}

\subsection{Limitations}

\subsubsection{Data Collection and Cleaning}

There are two primary limitations concerning the data collection and cleaning process used in this study. The first involves the erratic and transitory nature of emotion which may affect the consistency of the responses. My concern is that the emotions I am trying to capture will diffuse or transform throughout the questioning process (Kim \& Kanfer, 2009). By reducing the number of questions presented to participants in each trial I expect to mitigate this effect as well as reduce cognitive fatigue or boredom effects that may confound the results.

The second limitation concerns the data cleaning process. As with any study of this nature, there is a degree of comprehension issues that lead participants to identify strictly dominated responses as their indifference point. These are easily identifiable and excluded from the analyses, however, the likelihood of providing illogical answers is greater if participants did not follow a decision rule. Several participants, for example, identified answers that equalized the expected value of the gambles as their indifference point which does not result in any strictly dominated responses. This may lead to a disproportionate number of participants following a decision rule in the final dataset thereby mitigating the effect of the regressors on the dependent variables and biasing the results towards linearity. This is confirmed in a follow-up analysis that ran the same OLS regression models on only those who did not identify the expected value as their indifference point. The results showed that the signs of the significant determinants remain the same, but the magnitude and significance of their impact on the model parameters increased.

\subsubsection{Elicitation Methods}

One limitation to the TO method used to elicit individual utilities in the gain or loss domains is known as error propagation. Error propagation occurs in all chaining methods where a response to one question is used to elicit a response to another. The propagation of these errors occurs when the random error in one inferred utility value affects that in another. This means that the magnitude of certain error terms is an increasing function of the number of elicited utility points (Wakker \& Deneffe, 1996). This affects the variance of the utility function over the standard 
outcome sequence with the greatest distortion occurring in the median member of the standard sequence (Blavatskyy, 2006).

Abdellaoui's (2000) decision weight elicitation method has two main limitations. First, it requires decision makers to choose an outcome in the first stage and a probability in the second stage meaning there is a difference in the response scale which may not be compatible. Second, any errors inherent in the TO method carry over and are combined with the randomly occurring errors when making probability inferences (Abdellaoui, 2000; Blavatskyy, 2006). This is because decision weights are calculated from the residual after utility effects have been removed and means that the reliability of the decision weight measurements depend on the reliability of the utility measurements. This can be problematic since decision weights are calculated as a ratio and even very small errors in the numerator can produce large errors in the quotient if the denominator is small (Bleichrodt \& Pinto, 2000).

\subsubsection{Compensation}

Participants in this study were not compensated financially for their participation which presents a significant caveat. Many believe that monetary incentives are needed to motivate participants to choose the gamble they feel is in their best interest and to provide a direct link between choices and outcomes. Battalio, Kagel \& Jiranyakul (1990), for example, found that participants facing real payoffs were significantly more risk averse than those facing hypothetical sums and advise caution in interpreting the results of such experiments. One rationale for these criticisms stems from the tendency for people to economize decision costs. The argument is that when money is involved people are more willing to expend the mental effort to make sure they are making the right decision.

While the issue of incentives remains an ongoing debate, there is also evidence to show that participant responses are unaffected by financial incentives. The argument is that participants have no reason to misrepresent their choice preferences and there is a growing body of evidence to support this proposition. Camerer (1989) tested different payment conditions and found that "Subjects who actually played a gamble were no more reliable than subjects who did not play, and they took the same amount of time making choices" (p. 82). Tversky and Kahneman (1992) also chose to use hypothetical sums in their seminal paper CPT stating that, from their 
experience, they did not find any differences in the behaviour of participants to justify playing out the gambles.

Additionally, loss aversion, the common consequence effect, the common ratio effect, source dependence, and preference reversals have been observed under both hypothetical and real sums. Grether \& Plott (1979) conducted a series of experiments to explain the preference reversal phenomenon using economic theories. One theory, the misspecified incentives theory, asserts that subjects will not take seriously decisions that have no real consequences. The misspecified incentives theory along with a dozen others economic explanations for preference reversals were controlled for in the experiments and rejected as a cause of preference reversal. In fact, the authors found that preference reversals were more pervasive in experiments that provided monetary incentives.

It is increasingly common in the literature for studies involving lottery-style gambles to use hypothetical sums, including several similar studies outlined in the literature review (CamposVazquez \& Cuilty, 2014; Hockey et al. 2000; Callen et al. 2014; Guiso et al. 2013). One reason why hypothetical sums are preferred in this experiment relates to the design of the elicitation method. Because this elicitation method uses chained questions, a clever participant may state a higher indifference outcome in the first gain gamble so that each subsequent question has a higher expected value. This is one drawback of chained methods that is effectively mitigated by providing fixed incentives or hypothetical sums.

\subsection{Future Work}

The significance of visceral states and their interactions outlined in this study support the growing contention that visceral states are a primary driver of decision making. The area of inquiry, however, is relatively undeveloped and more studies are needed generalize these conclusions to different populations and to different areas such as health care. I discuss briefly one avenue for future research based on my findings in this thesis research.

One of my findings suggests that personality traits, namely neuroticism and contentiousness, play a significant role on risk preference through their interaction with several of the visceral factors explored in this study. The risk preference of participants exhibiting high levels of neuroticism, for example, was found to be less affected by anger, sadness, anxiety and happiness. 
This was surprising since people who exhibit this personality trait are believed to react more strongly to many of the visceral factors under investigation (Kehoe et al. 2012). Interaction effects are found between neuroticism and emotions for each of the risk preference parameters under consideration and the interaction consistently acts to moderate the effect of the most strongly related emotions on each of the parameters of interest.

Additional research using more comprehensive personality trait assessments is needed to confirm these findings and understand the nature of this interaction. It is possible that the relationship between these effects and personality traits may provide greater insight into the mechanisms underlying the effects of visceral factors on behaviour and expose techniques to moderate the adverse effects of visceral factors on decision making. Additionally, the relationship between visceral factors and stable personality traits may be of practical benefit in identifying those who are more prone to irrational behaviour resulting from an increased susceptibility to the effects of visceral factors. 


\section{APPENDICES}

\section{Appendix A - Expected Utility Theory: Axiomatic Foundation}

\section{$\geqslant$ Follows Weak Ordering}

Transitivity: if $X \geqslant Y$ and $Y \geqslant Z$ then $X \geqslant Z$

Completeness: for all $X, Y ; X \geqslant Y$ and/or $Y \geqslant X$

\section{Continuity:}

For any gamble $G 2$ that satisfies $G 1<G 2<G 3$ there exists $G 2 \sim q G 3+(1-q) G 1$

Independence:

$X \geqslant Y$ implies $q X+(1-q) Z \geqslant q Y+(1-q) Z$ for all $q \in(0,1]$

\section{Axiom Implications}

Betweenness: (Implied by Independence)

If $X>Y$ then $X>p X+(1-p) Y>Y$ for all $p \in(0,1)$

Consequence Monotonicity:

$X p Y \prec X^{\prime} p Y$ when $X^{\prime}>X$

\section{Probability Monotonicity:}

$X p Y \prec X(p+q) Y$ when $X>Y$ and $q>0$

\section{Idempotence:}

$G=\left(x, p_{1} ; x, p_{2} ; \ldots ; x, p_{n}\right) \sim x$

\section{Coalescing:}

$G=(x, p ; x, q ; y, 1-p-q) \sim G=(x, p+q ; y, 1-p-q)$, where $x>y>0$ or $y>x>0$

\section{Appendix B - Stable Determinants of Risk Preference}

1. Age [discrete]

2. Education [Some high school, High school, Some college/University, Post-secondary degree or certificate, Post-graduate]

3. Experience investing $[\mathrm{Y} / \mathrm{N}]$

4. Employment status [Part-time, Full-time, Student, Retired, Not Employed]

5. Occupation [Self-employed, working professional]

6. Yearly income [Continuous]

7. Combined income [Continuous]

8. Gross wealth [Continuous]

9. Net wealth [Continuous]

10. Years until retirement $[0-9,10-19,20+]$ 
11. Gender [Male, Female]

12. Race [Black, White, Hispanic, Asian, Other]

13. Marital Status [Married, Couple, Single]

14. Head of household [Y/N]

15. Number of dependents [Discrete]

16. Number of people in household [Discrete]

\section{Appendix C - Illustration of the Survey Presented to Participants}

Figure 14 - Illustration of the Survey Presented to Participants
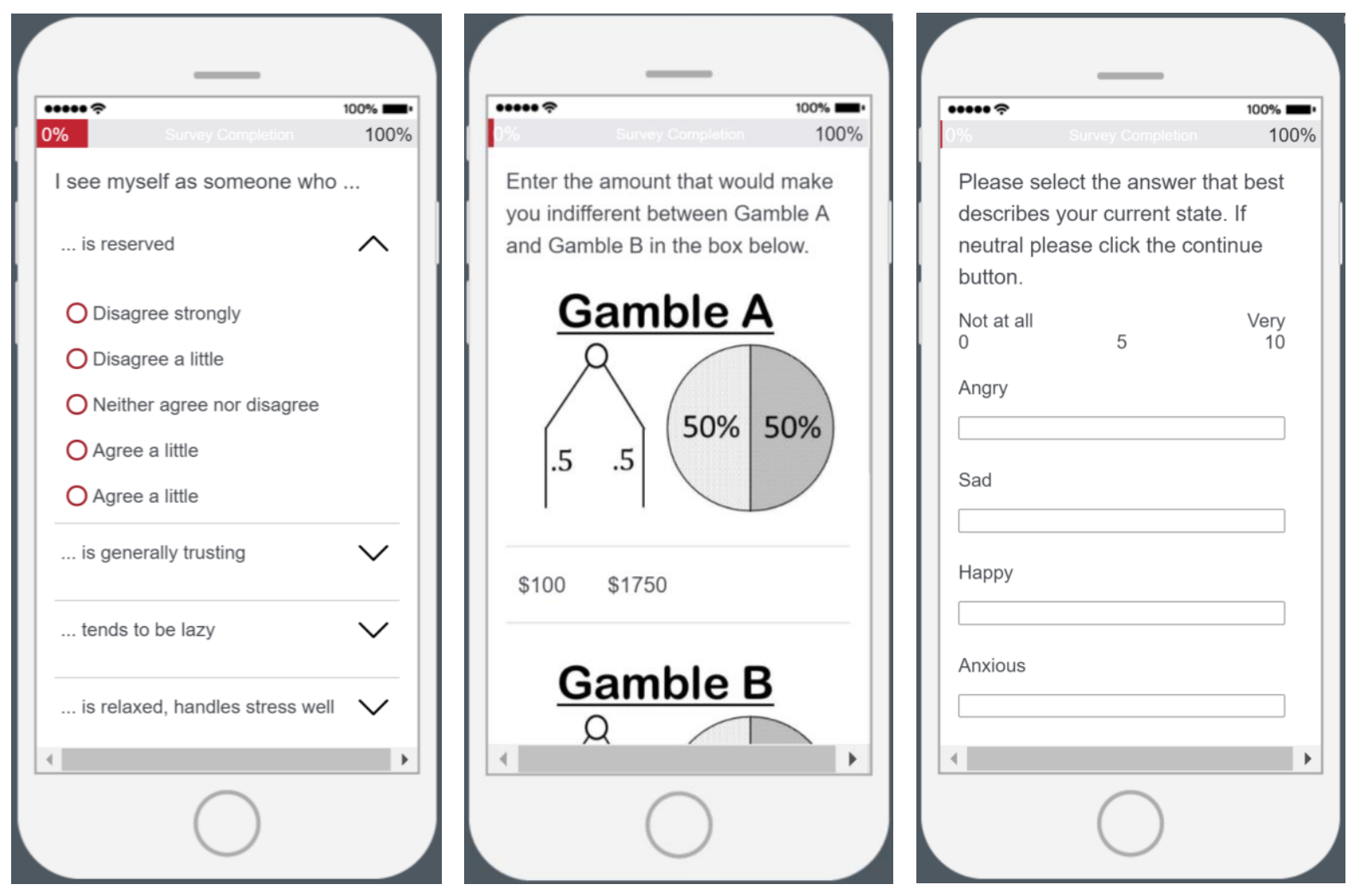


\section{REFERENCES}

Abdellaoui, M. (2000). Parameter-Free Elicitation of Utility and Probability Weighting Functions. Management Science, Vol. 46, No. 11, pp. 1497-1512.

Abdellaoui, M., Bleichrodt, H. \& L'Haridon, O. (2008). A tractable method to measure utility and loss aversion under prospect theory. Journal of Risk and Uncertainty, Vol 36, pp. 245266.

Abdellaoui, M., Bleichrodt, H., L’Haridon, O. \& van Dolder, D. (2016). Measuring Loss Aversion under Ambiguity: A Method to Make Prospect Theory Completely Observable. Journal of Risk and Uncertainty, Vol. 52, pp. 1-20.

Abdellaoui, M., Bleichrodt, H. \& Paraschiv, C. (2007). Loss Aversion under Prospect Theory: A Parameter-Free Measurement. Management Science, Vol. 53, No. 10, pp. 1659-1674.

Abdellaoui, M., Vossmann, F. \& Weber, M. (2005). Choice-Based Elicitation and Decomposition of Decision Weights for Gains and Losses under Uncertainty. Management Science. Vol. 51, No. 9, pp. 1384-1399.

Agrawal, U., Giripunje, S. \& Bajaj, P. (2013). Emotion and Gesture Recognition with Soft Computing Tool for Drivers Assistance System in Human Centered Transportation. IEEE International Conference on Systems Man and Cybernetics.

Alchian, A.A. (1953). The Meaning of Utility Measurement. The American Economic Review, Vol. 43, No. 1, pp. 26-50.

Alarié, Y. \& Dionne, G. (2001). Lottery decisions and probability weighting function. Journal of Risk and Uncertainty, 22, 21-33.

Allais, M. (1953). Le comportement de l'homme rationnel devant le risque: Critique des postulats et axiomes de l'école Américaine. Econometrica, Vol. 21, No. 4.

Allais, M. (1979). The so-called Allais Paradox and rational decision under uncertainty, in Expected Utility Hypotheses and the Allais Paradox, ed. by Maurice Allais and Ole Hagen. Dordrecht, Holland: Reidel, 437-481. 
Andersen, S., Harrison, G.W., \& Rutström, E. E. (2006). Dynamic choice behaviour: asset integration and natural reference points. Working paper, 06-07, Department of Economics, College of Business Administration, University of Central Florida.

Andrade, E.B., Odean, T. \& Lin, S. (2016). Bubbling with Excitement: An Experiment. Review of Finance, pp. 447-466, doi: 10.1093/rof/rfv016.

Ball, G. \& Breese, J. (2000). Emotion and Personality in a Conversational Agent. In J. Cassell, J. Sullivan, E. Churchill \& S. Prevost (Eds.), Embodied Conversational Agents (pp. 189-219). Cambridge, MA: MIT Press.

Batchelder, W.H. (2010). Mathematical psychology. Wiley Interdisciplinary Reviews: Cognitive Science, Vol. 1, No. 5, pp. 759-765.

Battalio, R.C., Kagel, J.H. \& Jiranyakul, K. (1990). Testing Between Alternative Models of Choice Under Uncertainty: Some Initial Results. Journal of Risk and Uncertainty, Vol. 3, pp. $25-50$.

Barkan, R. \& Busemeyer, J.R. (2003). Modeling Dynamic Inconsistency with a Changing Reference Point. Journal of Behavioral Decision Making, Vol. 16, pp. 235-255.

Bernoulli, D. ([1738] 1954). Exposition of a New Theory on the Measurement of Risk. Econometrica, 22, pp. 23-36.

Bechara, A., Damasio, H., Tranel, D. \& Damasio, A.R. (1997). Deciding advantageously before knowing the advantageous strategy. Science, Vol. 275, pp. 1293-1295.

Becker, J.L. \& Sarin, R.K. (1987). Lottery Dependent Utility. Management Science, Vol. 33, No. 11, pp. 1367-1382.

Bell, D.E. (1982). Regret in Decision Making under Uncertainty. Operations Research, Vol. 30, No. 5, pp. 961-98.

Bell, D.E. (1985). Disappointment in Decision Making under Uncertainty. Operations Research, Vol. 33, No. 1, pp. 1-2.

Benartzi, S. \& Thaler, R.H. (1995). Myopic Loss Aversion and the Equity Premium Puzzle. The Quarterly Journal of Economics, Vol. 110, No. 1, pp. 73-92. 
Bendahan, S., Goette, L., Thoresen, J., Loued-Khenissi, L., Hollis, F. \& Sandi, C. (2017). Acute stress alters individual risk taking in a time dependent manner and leads to anti-social risk. European Journal of Neuroscience, Vol. 45, pp. 877-885.

Benet-Martinez, V. \& John, O.P. (1998). Los Cinco Grandes Across Cultures and Ethnic Groups: Multitrait Multimethod Analyses of the Big Five in Spanish and English. Journal of Personality and Social Psychology, Vol. 75, No. 3. 729-750.

Barnard, C.J. \& Brown, C.A.J. (1985). Risk-Sensitive Foraging in Common Shrews (Sorex araneus L.). Behavioral Ecology and Sociobiology, Vol. 16, No. 2, pp. 161-164.

Binswanger, H. (1981). Attitudes toward risk: Theoretical implications of an experiment in rural India. The Economic Journal, 9I, 867-890.

Birnbaum, M.H. (2005). A Comparison of Five Models that Predict Violations of First-Order Stochastic Dominance in Risky Decision Making. The Journal of Risk and Uncertainty, 31:3; $263-287$.

Birnbaum, M.H. \& Navarrete, J.B. (1998). Testing Descriptive Utility Theories: Violations of Stochastic Dominance and Cumulative Independence. Journal of Risk and Uncertainty, 17:49-78.

Birnbaum, M.H., Patton, J.N. \& Lott, M.K. (1999). Evidence against Rank-Dependent Utility Theories: Tests of Cumulative Independence, Interval Independence, Stochastic Dominance, and Transitivity. Organizational Behavior and Human Decision Processes, Vol. 77, No. 1, pp. 44-83.

Blavatskyy, P. (2006). Error propagation in the elicitation of utility and probability weighting functions. Theory and Decision, 60: 315-334.

Bleichrodt, H. \& Pinto J.L. (2000). A Parameter-Free Elicitation of the Probability Weighting Function in Medical Decision Analysis. Management Science. Vol. 46, No. 11, pp.14851496.

Booij, A.S. (2009). Essays on the measurement sensitivity of risk aversion and causal effects in education. Amsterdam: Thela Thesis. 
Booij, A.S., Bernard M.S. van Praag \& van de Kuilen, G. (2009). A parametric analysis of prospect theory's functionals for the general population. Theory and Decision, Vol. 68, pp. $115-148$.

Booij, A.S. \& van de Kuilen, G. (2009). A Parameter-Free Analysis of the Utility of Money for the General Population under Prospect Theory. Journal of Economic Psychology, 30(4), pp. 651-666.

Brayman, S., Finke, M., Bessner, E., Bessner, B., Grable, J., Griffin, P. \& Clement, R. (2015). Current Practices for Risk Profiling in Canada And Review of Global Best Practices. PlanPlus Inc., Prepared for the Investor Advisory Panel of the Ontario Securities Commission.

Broekens, J. \& Brinkman, W-P. (2013). Affect Button: a method for reliable and valid affective self-report. Retrieved from: http://mmi.tudelft.nl/ joostb/files/Broekens\%20and\%20Brinkman\%202013. pdf

Buckert, M., Schwieren, C., Kudielka, B.M. \& Fiebach, C.J. (2014). Acute stress affects risk taking but not ambiguity aversion. Decision Neuroscience, Vol. 8, No. 82.

Cahlíková, J. \& Cingl, L. (2017). Risk preferences under acute stress. Experimental Economics, Vol. 20, pp. 209-236, DOI 10.1007/s10683-016-9482-3

Cai, H. \& Lin, Y. (2007). An Experiment to Non-Intrusively Collect Physiological Parameters towards Driver State Detection. SAE Technical Paper, 2007-01-0403, https://doi.org/10.4271/2007-01-0403.

Callen, M., Isaqzadeh, M., Long, J. D. \& Sprenger, C. (2014). Violence and risk preference Experimental evidence from Afghanistan. American Economic Review, 104(1): 123-148.

Camerer, C. (1989). An Experimental Test of Several Generalized Utility Theories. Journal of Risk and Uncertainty, Vol. 2, pp. 61-104.

Camerer, C. (1998). Bounded Rationality in Individual Decision Making. Experimental Economics, Vol. 1, pp. 163-183. 
Camerer, C. (1999). Behavioral economics: Reunifying psychology and economics. Proceedings of the National Academy of Sciences of the United States of America, Vol. 96, pp. 1057510577.

Camerer, C. (2005). Three Cheers - Psychological, Theoretical, Empirical - For Loss Aversion. Journal of Marketing Research, Vol. 42, No. 2, pp. 129-133.

Camerer, C.F. \& Hogarth, R.M. (1999). The effects of financial incentives in experiments: A review and capital-labor-production framework. Journal of Risk and Uncertainty, Vol. 19, pp. $7-42$.

Cameron, L. \& Shah, M. (2015). Risk-Taking Behavior in the Wake of Natural Disasters. The Journal of Human Resources, Vol. 50, No. 2.

Campbell, J. \& Ehlert, U. (2012). Acute psychosocial stress: Does the emotional stress response correspond with physiological responses? Psychoneuroendocrinology, Vol. 37, pp. 11111134.

Campos-Vazquez, R.M. \& Cuilty, E. (2014). The role of emotions on risk aversion: A Prospect Theory experiment. Journal of Behavioral and Experimental Economics. Vol. 50, pp. 1-9.

Carr, K.D. (1996). Feeding, drug abuse, and the sensitization of reward by metabolic need. Neurochemical Research, Vol. 21, No. 11, pp. 1455-1467.

Chang, C., DeVaney S. \& Chiremba S.T. (2004). Determinants of Subjective and Objective Risk Tolerance. Research \& Theory. Volume 3, Issue 3, pp. 53-67

Chang, E.C., Luo, Y. \& Ren, J. (2013). Pricing deviation, misvaluation comovement, and macroeconomic conditions. Journal of Banking \& Finance, Vol. 37, pp. 5285-5299.

Cohen, M., Jaffray, J. \& Said, T. (1987). Experimental Comparison of Individual Behavior under Risk and under Uncertainty for Gains and for Losses. Organizational Behaviour and Human Decision Processes, 39, 1-22.

Conte, A., Levati, M.V. \& Nardi, C. (2016). Risk Preferences and the Role of Emotions. Economica, doi:10.1111/ecca.12209 
Costa, P.T., \& McRae, R.R. (1992). Revised NEO Personality Inventory (NEO-PI-R) and NEO Five-Factor Inventory (NEO-FFI) professional manual. Odessa, Florida: Psychological Assessment Resources, Inc.

Critchley, H.D. \& Harrison, N.A. (2013). Visceral Influences on Brain and Behavior. Neuron, Vol. 77, No. 4, pp. 624-638

Croson, R. \& Gneezy, U. (2009). Gender Differences in Preferences. Journal of Economic Literature, Vol. 47, No. 2, pp. 448-474

Daveya, H.M., Barratta, A.L., Butowb, P.N. \& Deeks, J.J. (2007) A one-item question with a Likert or Visual Analog Scale adequately measured current anxiety. Journal of Clinical Epidemiology, Vol. 60, pp. 356360.

De Bondt, W.F.M. \& Thaler, R. (1984). Does the Stock Market Overreact? The Journal of Finance, Vol. 40, No. 3, Papers and Proceedings of the Forty-Third Annual Meeting American Finance Association, Dallas, Texas, December 28-30, 1984 (Jul.,1985), pp. 793805.

De Bondt, W.F.M. \& Thaler, R. (1987). Further Evidence on Investor Overreaction and Stock Market Seasonality. The Journal of Finance, Vol. 42, No. 3, Papers and Proceedings of the Forty-Fifth Annual Meeting of the American Finance Association, New Orleans, Louisiana, December 28-30, 1986 (Jul. 1987), pp. 557-581.

DellaVigna, S. (2009). Psychology and Economics: Evidence from the Field. Journal of Economic Literature, Vol. 47, No. 2, pp. 315-372.

Dinges, D.F., Pack, F., Williams, K., Gillen, K.A., Powell, J.W., Ott, G.E., Aptowicz, C. and Pack, A.I. (1997). Cumulative sleepiness, mood disturbance, and psychomotor vigilance performance decrements during a week of sleep restricted to 4-5 hours per night. Sleep, Vol. 20, pp. 267-277.

Ditto, P.H., Pizarro, D.A., Epstein, E.B., Jacobson, J.A. \& MacDonald, T.K. (2006). Visceral Influences on Risk-Taking Behavior. Journal of Behavioral Decision Making, Vol. 19, pp. 99-113. 
Donaldson, T.S. (1968). Robustness of the F-test to errors of both kinds and the correlation between the numerator and denominator of the F-ratio. Journal of the American Statistical Association, Vol. 63, pp. 660-676.

Dunn, L.F. (1996). Loss Aversion and Adaption in the Labour Market: Empirical Indifference Functions and Labour Supply. Review of Economics and Statistics, Vol. 78, pp. 441-450.

Eckel, C.C., El-Gamal, M.A. \& Wilson, R.K. (2009). Risk loving after the storm: A BayesianNetwork study of Hurricane Katrina evacuees. Journal of Economic Behavior \& Organization, Vol. 69, pp. 110-124.

Eckel, C.C. \& Grossman, P.J. (2002). Sex differences and statistical stereotyping in attitudes toward financial risk. Evolution and Human Behavior, Vol. 23, pp. 281-295.

Eckel, C.C. \& Grossman, P.J. (2008). Forecasting risk attitudes: An experimental study using actual and forecast gamble choices. Journal of Economic Behavior \& Organization, Vol. 68, No. 1, pp. 1-17.

Ekkekakis, P. (2012). Affect, Mood, and Emotion. In: Tenenbaum, G., Eklund, R.C. \& Kamata, A. (2012). Measurement in sport and exercise psychology. Human Kinetics, ISBN-1O: 07360-8681-1

Ellsberg, D. (1961). Risk Ambiguity, and the Savage Axioms. The Quarterly Journal of Economics, Vol. 75, No. 4, pp. 643-669.

Etchart-Vincent, N. (2004). Is probability weighting sensitive to the magnitude of consequences? An experimental investigation on losses. Journal of Risk and Uncertainty, Vol. 28, pp. 217235.

Etchart-Vincent, N. (2009). Probability weighting and the 'level' and 'spacing' of outcomes: An experimental study over losses. Journal of Risk \& Uncertainty, Vol 39, pp. 45-63.

Eyben, F., Wöllmer, M., Poitschke, T., Schuller, B., Blaschke, C., Färber, B., \& Nguyen-Thien, N. (2010). Emotion on the road--necessity, acceptance, and feasibility of affective computing in the car. Advances in Human - Computer Interaction, 2010 doi:http://dx.doi.org.ezproxy.lib.ryerson.ca/10.1155/2010/263593 
Farquhar, P. (1984). Utility Assessment Methods. Management Science, Vol. 30, No. 11.

Fehr-Duda, H., Epper, T., Bruhin, A. \& Schubert, R. (2011). Risk and Rationality: The effects of mood and decision rules on probability weighting. Journal of Economic Behaviour \& Organization, Vol. 78, pp. 14-24.

Fehr-Duda, H., de Gennaro, M., \& Schubert, R. (2006). Gender, financial risk and probability weights. Theory and Decision, 60, 283-313.

Ferrara, M., Bottasso, A., Tempesta, D., Carrieri, M., De Gennaro, L. \& Ponti, G. (2015). Gender Differences in Sleep Deprivation Effects on Risk and Inequality Aversion: Evidence from an Economic Experiment. PLoS One, DOI:10.1371/journal.pone.0120029

Figlewicz, D.P., Naleid, A.M.D. \& Sipols, A.J. (2007). Modulation of food reward by adiposity signals. Physiology \& behavior, Vol. 91, pp. 473-478.

Finucane, M.L., Alhakami, A., Slovic, P. \& Johnson, S.M. (2000). The Affect Heuristic in Judgments of Risks and Benefits. Journal of Behavioral Decision Making, Vol. 13, pp. 1-17.

Fong, T, Nourbakhsh, I. \& Dautenhahn, K. (2003). A survey of socially interactive robots. Robotics and Autonomous Systems, Vol. 42, No. 2-3 143-166.

Forgas, J.P. (1995). Mood and Judgment: The Affect Infusion Model (AIM). Psychological Bulletin, Vol. 117, No. 1, pp. 39-66.

Friedman, M. \& Savage, L.J. (1948). The Utility Analysis of Choices Involving Risk. The Journal of Political Economy, Vol. 56, No. 4, pp. 279-304.

Gibson, R., Michayluk, D. \& Van de Venter, G. (2013). Financial risk tolerance: An analysis of unexplored factors. Financial Services Review. Spring 2013; 22, 1.

Gintis, H. (2011). The future of behavioural game theory. Mind \& Society, Vol. 10, pp. 97-102. DOI 10.1007/s11299-011-0091-8. Springer-Verlag.

Goldberg, L.R., Johnson, J.A., Eber, H.W., Hogan, R., Ashton, M.C., Cloninger, C.R., \& Gough, H.C. (2006). The International Personality Item Pool and the future of public-domain personality measures. Journal of Research in Personality, Vol. 40, pp. 84-96. 
Goldstein, W.M. \& Einhorn, H. J. (1987). Expression theory and the preference reversal phenomena. Psychological Review, Vol. 94, pp. 236-254.

Gosling, S.D., Rentfrow, P.J. \& Swann Jr, W.B. (2003). A very brief measure of the Big-Five personality domains. Journal of Research in Personality, Vol. 37, pp. 504-528.

Gonzalez, R. \& Wu, G. (1999). On the Shape of the Probability Weighting Function. Cognitive Psychology, Vol. 38, pp. 129-166.

Grable, J. E. (2000). Financial risk tolerance and additional factors that affect risk taking in everyday money matters. Journal of Business and Psychology, Vol. 14, No. 4, Summer 2000.

Grable, J. E., \& Joo, S. (2004). Environmental and biopsychosocial factors associated with risk tolerance. Financial Counseling and Planning, Vol. 15, pp. 73-82

Grable, J.E. \& Roszkowski, M.J. (2008). The influence of mood on the willingness to take financial risks. Journal of Risk Research, Vol. 11, No. 7, pp. 905-923.

Grether, D.M. \& Plott, C.R. (1979). Economic Theory of Choice and the Preference Reversal Phenomenon. The American Economic Review, Vol. 69, No. 4, pp. 623-638

Guiso, L., Sapienza, P. \& Zingales, L. (2013). Time varying risk aversion. NBER Working Paper Series, Working Paper 19284, http://www.nber.org/papers/w19284

Gul, F. (1991). A Theory of Disappointment Aversion. Econometrica, Vol. 59, No. 3, pp. 667686.

Halek, M. \& Eisenhauer, J. G. (2001). Demography of Risk Aversion. The Journal of Risk and Insurance. Vol. 68, No. 1, pp. 1-24

Hallahan, T. A., Faff, R. W. \& McKenzie, M. D. (2004). An empirical investigation of personal financial risk tolerance. Financial Services Review, Vol. 13, No. 1.

Hanna, S. \& Chen, P. (1997). Subjective and Objective Risk Tolerance: Implications for Optimal Portfolios. Financial Counseling and Planning, Vol. 8, No. 2, pp. 17-26.

Harbaugh, W. T., Krause, K., \& Vesterlund, L. (2002). Risk attitudes of children and adults: choices over small and large probability gains and losses. Experimental Economics, 5, 53-84. 
Hardie, B.G., Johnson, G.S. \& Fader, P.S. (1993). Modeling Loss Aversion and Reference Dependence Effects on Brand Choice. Marketing Science, Vol. 12, No. 4, pp. 378-394.

Harless, D. \& Camerer, C. (1994). The Predictive Utility of Generalized Expected Utility Theories. Econometrica, Vol. 62, No. 6, pp. 1251-1289.

Harrison, Y. \& Horne, J.A. (2000). The Impact of Sleep Deprivation on Decision Making: A Review. Journal of Experimental Psychology: Applied, Vol. 6, No. 3, pp. 236-249.

Harrison, G.W., \& Rutström, E.E. (2009). Expected utility theory and prospect theory: One wedding and a decent funeral. Experimental Economics, Vol. 12, pp. 133-158.

Hawley, C. B. \& Fujii, E.T. (1993). An empirical analysis of preferences for financial risk: Further evidence on the Friedman-Savage model. Journal of Post Keynesian Economics.

Heath, C., Larrick, R.P. \& Wu G. (1999). Goals as Reference Points. Cognitive Psychology, 38, 79-109

Hershey, J.C. \& Schoemaker, P.J.H. (1980). Risk Taking and Problem Context in the Domain of Losses: An Expected Utility Analysis. The Journal of Risk and Insurance, Vol. 47, No. 1, pp. $111-132$.

Hey, J.D. (2001). Does repetition improve consistency? Experimental Economics, Vol. 4, No. 1, pp. 5-54.

Hey, J.D. \& Orme, C. (1994). Investigating Generalizations of Expected Utility Theory Using Experimental Data. Econometrica, Vol. 62, No. 6, pp. 1291-1326.

Hirshleifer, D. \& Shumway, S. (2003). Good Day Sunshine: Stock Returns and the Weather. The Journal of Finance, Vol. 58, No. 3, pp. 1009-1032.

Hockey, G.R.J., Maule, A.J., Clough, P.J. \& Bdzola, L. (2000). Effects of negative mood states on risk in everyday decision making. Cognition and Emotion. Vol. 14, No. 6, pp. 823-855.

Hoeger Bement, M., Weyer, A., Keller, M., Harkins, A.L. \& Hunter, S.K. (2010). Anxiety and stress can predict pain perception following a cognitive stress. Physiology \& Behavior, Vol. 101, pp. 87-92. 
Holt, C. (1986). Preference Reversals and the Independence Axiom. The American Economic Review. Vol. 76, No. 3, pp. 508-515.

Holt, C.A. \& Laury, S.K. (2002). Risk Aversion and Incentive Effects. The American Economic Review, Vol. 92, No. 5, pp. 1644-1655

Horst, P. (1932). A Method for Determining the Absolute Affective Value of a Series of Stimulus Situations. The Journal of Educational Psychology.

Hudlicka, E. (2009). Affective game engines: motivation and requirements. Proceedings of the 4th International Conference on Foundations of Digital Games, pp. 299-306.

Humphrey, S. J., \& Verschoor, A. (2004). The probability weighting function: Experimental evidence from Uganda, India and Ethiopia. Economics Letters, Vol. 84, pp. 419-425.

Isen, A.M. \& Patrick, R. (1983). The Effect of Positive Feelings on Risk Taking: When the Chips Are Down. Organizational Behavior and Human Performance, Vol. 31, pp. 19-202.

Isrealson, D. (2016). Automated Investing: Domestic Choice, the robo-adviser choices grow in Canada. The Globe and Mail, Published Saturday, Jun. 18, 2016 5:00AM EDT

Jaeschke, R., Singer, J. \& Guyatt, G.H. (1990). A Comparison of Seven-Point and Visual Analogue Scales: Data from a Randomized Trial. Controlled Clinical Trials, Vol. 11, pp. 4351.

Jolliffe, I.T. (1972). Discarding variables in a principal component analysis. I. Artificial data. Journal of Applied Statistics, Vol. 21, pp. 160-173.

Jolliffe, I.T. (1986). Principal Component Analysis. New York: Springer

Jouini, E. \& Napp, C. (2010). Unbiased Disagreement in Financial Markets, Waves of Pessimism and the Risk-Return Trade-off. Review of Finance, Vol. 15, No. 3, pp. 1-27.

Kahneman, D., Knetsch, J.L. \& Thaler, R.H. (1991). Anomalies: The Endowment Effect, Loss Aversion, and Status Quo Bias. Journal of Economic Perspectives, 5(1): 193-206

Kahneman D. \& Tversky A. (1979). Prospect Theory: An Analysis of Decision Under Risk. Econometrica, 47 (2), 264-291. 
Kahneman D. \& Tversky A. (1984). Choices, Values and Frames. American Psychologist. Vol. 39, No. 4, 341-35.

Kaiser, H.F. (1960). The application of electronic computers to factor analysis. Educational and Psychological Measurement, Vol. 20, pp. 141-151.

Karmarkar, U. (1978). Subjectively weighted utility: A descriptive extension of the expected utility model. Organizational Behavior and Human Performance. 21, 61-72.

Keeney, R. \& Raiffa, H. (1976). Decisions with Multiple Objectives: Preferences and Value Tradeoffs. Wiley. New York.

Kehoe, E.G., Toomey, J.M., Balsters, J.H. \& Bokde, A.L.W. (2012). Personality modulates the effects of emotional arousal and valence on brain activation. Social Cognitive and Affective Neuroscience, Vol. 7, No. 7, pp. 858-870.

Kern, S., Oakes, T.R., Stone, C.K., McAuliff, E.M., Kirschbaum, C. \& Davidson, R.J. (2008). Glucose metabolic changes in the prefrontal cortex are associated with HPA axis response to a psychosocial stressor. Psychoneuroendocrinology, Vol. 33, pp. 517-529.

Killgore, W.D.S., Balkin, T.J. \& Wesensten, N.J. (2006). Impaired decision making following 49 $\mathrm{h}$ of sleep deprivation. Journal of Sleep Research, Vol. 15, pp. 7-13.

Kim, M.Y. \& Kanfer, R. (2009). The joint influence of mood and a cognitively demanding task on risk-taking. Motivation and Emotion, Vol. 33, pp. 362-372, DOI 10.1007/s11031-009$9147-\mathrm{z}$

Kirschbaum, C., Pirke, K.-Ml. \& Hellhammer, D.H. (1995). Preliminary evidence for reduced cortisol responsivity to psychological stress in women using oral contraceptive medication. Psychoneuroendocrinology, Vol. 20, pp. 509-514.

Kliger, D. \& Levy, O. (2003). Mood-induced variation in risk preferences. Journal of Economic Behavior \& Organization, Vol. 52, pp. 573-584.

Köbberling, V. \& Wakker, P.P. (2005). An index of loss aversion. Journal of Economic Theory, Vol. 122, pp. 119-131. 
Kothiyal, A., Spinu, V. \& Wakker P.P. (2014). An experimental test of prospect theory for predicting choice under ambiguity. Journal of Risk and Uncertainty, Vol. 48, pp. 1-17.

Krzysztofowicz, R. \& Duckstein, L. (1980). Strength of Preference and Risk Attitude in Utility Measurement. Organizational Behavior and Human Performance, Vol. 26, 326-348.

Kugler, T., Connolly, T. \& Ordóñez, L.D. (2012). Emotion, Decision, and Risk: Betting on Gambles versus Betting on People. Journal of Behavioral Decision Making, Vol. 25, pp. 123134.

Kuhlmann, T., Dantlgraber, M. \& Reips U-D. (2017). Investigating measurement equivalence of visual analogue scales and Likert-type scales in Internet-based personality questionnaires. The journal Behavior Research Methods, DOI 10.3758/s13428-016-0850-x

Kumar, A. \& Lee, C.M.C. (2006). Retail Investor Sentiment and Return Comovements. The Journal of Finance, Vol. 61, No. 5, pp. 2451-2487.

Lattimore, P.K., Baker, J.R., \& Witte, A.D. (1992). The influence of probability on risky choice: A parametric examination. Journal of Economic Behavior and Organization, Vol. 17, pp. 377-400.

Leith, K.P. \& Baumeister, R.F. (1996). Why Do Bad Moods Increase Self-Defeating Behavior? Emotion, Risk Taking, and Self-Regulation. Journal of Personality and Social Psychology, Vol. 71, No. 6, pp. 1250-1267.

Lerner, J.S. \& Keltner, D. (2000). Beyond valence: Toward a model of emotion-specific influences on judgement and choice. Cognition and Emotion, Vol. 14, No. 4, pp. 473-493.

Lerner, J.S. \& Keltner, D. (2001). Fear, Anger, and Risk. Journal of Personality and Social Psychology, Vol. 81. No. 1, pp. 146-159.

Lerner, J.S., Valdesolo, V. \& Kassam, K.S. (2015). Emotion and Decision Making. The Annual Review of Psychology, Vol. 66, pp. 799-823.

Lim, J., Wu, W., Wang, J., Detre, J.A., Dinges, D.F. \& Rao, H. (2010). Imaging brain fatigue from sustained mental workload: An ASL perfusion study of the time-on-task effect. NeuroImage, Vol., pp. 3426-3435. 
Litt, A., Eliasmith, C. \& Thagard, P. (2008). Neural affective decision theory: Choices, brains, and emotions. Cognitive Systems Research, Vol. 9, pp. 252-273.

Liu, C., Agrawal, P., Sarkar, N. \& Chen, S. (2009). Dynamic Difficulty Adjustment in Computer Games Through Real-Time Anxiety-Based Affective Feedback. International Journal of Human-Computer Interaction, Vol. 25, No. 6, pp. 506-529.

Lo, A. W., Repin, D. V., \& Steenbarger, B. N. (2005). Fear and greed in financial markets: A clinical study of day traders. Working paper.

Loewenstein, G. (1996). Out of Control: Visceral Influences on Behavior. Organizational Behavior and Human Decision Processes, Vol. 65, No. 3, pp. 272-292.

Loewenstein, G. (2000). Emotions in Economic Theory and Economic Behavior. The American Economic Review, Vol. 90, No. 2, pp. 426-432.

Loewenstein, G., Weber, EU., Hsee, CK. \& Welch, N. (2001). Risk as feelings. Psychological Bulletin, Vol. 127, No. 2, 267-286.

Loomes, G. \& Sugden, R. (1982). Regret Theory: An Alternative Theory of Rational Choice Under Uncertainty. The Economic Journal, Vol. 92, No. 368, pp. 805-824.

Loomes, G. \& Sugden, R. (1986). Disappointment and Dynamic Consistency in Choice under Uncertainty. The Review of Economic Studies, Vol. 53, No. 2, pp. 271-282.

Loomes, G. \& Sugden, R. (1987). Testing for Regret and Disappointment in Choice Under Uncertainty. The Economic Journal, Vol. 97, Supplement: Conference Papers (1987), pp. $118-12$.

Lunney, G.H. (1970). Using Analysis of Variance with a dichotomous dependent variable: An empirical study. Journal of Educational Measurement, 7(4), 263-269.

Mallpress, D., Fawcett, T., Houston, A. \& McNamara, J. (2015). Risk Attitudes in a Changing Environment: An Evolutionary Model of the Fourfold Pattern of Risk Preferences. Psychological Review, 2015, Vol. 122, No. 2, 364-375. 
Mander, B.A., Reid, K.J., Davuluri, V.K., Small, D.M., Parrish, T.B., Mesulam, M., Zee, P.C. \& Gitelman, D.R. (2008). Sleep deprivation alters functioning within the neural network underlying the covert orienting of attention. Brain Research, Vol. 1217, pp. 148-156.

Markowitz, H. (1952). The Utility of Wealth. The Journal of Political Economy, Vol. 60, pp. $151-158$.

Markowitz, H. (1991). Foundations of Portfolio Theory. The Journal of Finance, Vol. 46, No. 2 , pp. $469-477$.

McCord, M. \& de Neufville, R. (1986). Lottery Equivalents': Reduction of the certainty effect problem in utility assessment. Management Science. 32, pp. 56-60.

McKenna, B.S., Dickinson, D.L., Orff, H.J. \& Drummond, S.P.A. (2007). The effects of one night of sleep deprivation on known-risk and ambiguous-risk decisions. Journal of Sleep Research. Vol. 16, pp. 245-252.

Mellers, B., Schwartz, A., Ho, K. \& Ritov, I. (1997). Decision Affect Theory: Emotional Reactions to the Outcomes of Risky Options. Psychological Science, Vol. 8, No. 6.

Mikolajczak, M., Roy, E., Luminet, O., Fille'e, C. \& De Timary, P. (2007). The moderating impact of emotional intelligence on free cortisol responses to stress.

Psychoneuroendocrinology, Vol. 32, pp. 1000-1012.

Mikolajczak, M., Roy, E., Luminet, O. \& de Timary, P. (2008). Resilience and hypothalamic pituitary - adrenal axis reactivity under acute stress in young men. Stress, Vol. 11, pp. 477482 .

Mistar, J. (2011). A Study of the Validity and Reliability of Self-Assessment. TEFLIN Journal, Vol. 22, No. 1.

Moscati I. (2013a). How cardinal utility entered economic analysis: 1909-1944. Euro. J. History of Economic Thought, Vol. 20, No. 6, pp. 906-939.

Moscati I. (2013b). Were Jevons, Menger, and Walras Really Cardinalists? On the Notion of Measurement in Utility Theory, Psychology, Mathematics, and Other Disciplines, 1870-1910. History of Political Economy 45:3 DOI 10.1215/00182702-2334740. 
Mosteller, F. \& Nogee, P. (1951). An experimental measurement of utility. The Journal of Political Economy. Oct. 1951, Vol. 59, No. 5.

Mullette-Gillman, O.A., Leong, R.L.F. \& Kurnianingsih, Y.A. (2015). Cognitive Fatigue Destabilizes Economic Decision Making Preferences and Strategies. PLoS One, DOI:10.1371/journal.pone.0132022

Noakes, S. (2016). How well does a financial adviser know your risk profile? Too many clientadviser disputes stem from poor assessment of risk tolerance, OSC says. CBC News. Posted: Jan 02, 2016 5:00 AM ET. http://www.cbc.ca/news/business/rrsp/osc-questionnaires-study1.3363957

Officer, R. \& Hatter, PI. N. (1968). Utility analysis in a practical setting. American Journal of Agricultural Economics, Vol. 50, No. 2, pp. 257-77.

Ombudsman for Banking Services and Investments (OBSI). (2014). 2014 Annual Report. Retrieved from https:/www.obsi.ca/en/download/fm/290/filename/Annual-Report-20141444055310-0ac88.pdf

Pareto, V. ([1898] 1966). Comment se pose le problème de l'économie pure. In Pareto 1966, pp. $102-109$.

Pareto, V. ([1909] 1971). Manual of Political Economy. New York: Kelley

Perraudin, W.R.M. \& Sorense, B.E. (2000). The demand for risky assets: Sample selection and household portfolios. Journal of Econometrics, Vol. 97, pp. 117-144.

Pilcher, J.J. \& Huffcutt, A.I. (1996). Effect of sleep deprivation on performance: A meta analysis. Sleep, Vol. 19, pp. 318-326.

Palma, A. \& Picard, N. (2010). Evaluation of MiFID questionnaires in France: Study for the Authorité des Marchés Financiers.

Porcelli, A.J. \& Delgado, M.R. (2009). Acute Stress Modulates Risk Taking in Financial Decision Making. Psychological Science, Vol. 20, No. 3.

Prelec, D. (1998). The Probability Weighting Function. Econometrica, Vol. 66, No. 3, pp. 497527. 
Quiggin, J. (1981). Risk Perception and the Analysis of Risk Attitudes. Australian Journal of Agricultural Economics. Vol. 25, No. 2, pp. 160-169.

Rabin, M. (1998). Psychology and Economics. Journal of Economic Literature, Vol. 36, pp. 1146.

Rammstedt, B. \& John, O.P. (2007). Measuring personality in one minute or less: A 10-item short version of the Big Five Inventory in English and German. Journal of Research in Personality, 41, 203-212.

Raspopow, K., Abizaid, A., Matheson, K. \& Anisman, H. (2010). Psychosocial stressor effects on cortisol and ghrelin in emotional and non-emotional eaters: influence of anger and shame. Hormones and Behavior, Vol. 58, pp. 677-684.

Ross, J.A. (2006). The Reliability, Validity, and Utility of Self-Assessment. Practical Assessment, Research \& Evaluation, Vol. 11, No. 10, ISSN 1531-7714.

Samuelson, P.A. (1938). The numerical representation of ordered classifications and the concept of utility. Review of Economic Studies, 6: 65-70.

Samuelson, W. \& Zeckhauser, R. (1988). Status Quo Bias in Decision Making. Journal of Risk and Uncertainty, Vol. 1, pp. 7-59

Sanfey, A.G. (2007). Decision Neuroscience: New Directions in Studies of Judgment and Decision Making. Current Directions in Psychological Science, Vol. 16, No. 3, pp. 151-155.

Saunders, E.M. (1993). Stock Prices and Wall Street Weather. The American Economic Review, Vol. 83, No. 5, pp. 1337-1345.

Schmeidler, D. (1989). Subjective Probability and Expected Utility without Additivity. Econometrica. Vol. 57, No. 3, pp. 571-58.

Schmidt, U. (1998). A Measurement of the Certainty Effect. Journal of Mathematical Psychology. Vol. 42, pp. 32-47.

Schmidt, U. (2000). The certainty effect and boundary effects with transformed probabilities. Economics Letters, Vol. 67, pp. 29-33. 
Schmidt, U. (2001). Lottery Dependent Utility: A Reexamination. Theory and Decision, Vol. 50, pp. 35-58, 2001.

Schmidt, U. (2002). An Experimental Test of Loss Aversion. The Journal of Risk and Uncertainty, Vol. 25, No. 3, pp. 233-249.

Scholten, M. \& Read, D. (2014). Prospect theory and the "forgotten" fourfold pattern of risk preferences. Journal of Risk and Uncertainty (2014) 48:67-83.

Shefrin, H. \& Statman, M. (1985). The disposition effect to sell winners too early and ride losers too long: Theory and evidence. Journal of Finance, Vol. 40, pp. 777-790.

Shiller, R.J. (2000). Irrational exuberance. Princeton, N.J.: Princeton University Press.

Shiv, B., Loewenstein, G., Bechara, A., Damasio, A., \& Damasio, H. (2005). Investment behavior and the dark side of emotion. Psychological Science, Vol. 16, pp. 435-439.

Simeon, D., Knutelska, M., Smith, L., Baker, B.R. \& Hollander, E. (2007). A preliminary study of cortisol and norepinephrine reactivity to psychosocial stress in borderline personality disorder with high and low dissociation. Psychiatry Research, Vol. 149, pp. 177-184.

Simon, H.A. (1956). Rational choice and the structure of environments. Psychological Review, Vol. 63, No. 2, pp. 129-138.

Simon, H.A. (1983). Reason in Human Affairs. Stanford, CA: Stanford Univ. Press.

Smith, C.A. \& Ellsworth, P.C. (1985). Patterns of Cognitive Appraisal in Emotion. Journal of Personality and Social Psychology, Vol. 48, No. 4, 813-83.

Starmer, C. (2000). Developments in Non-Expected Utility Theory: The Hunt for a Descriptive Theory of Choice under Risk. Journal of Economic Literature, Vol. 38, No. 2, pp. 332-382.

Starmer, C. \& Sugden, R. (1989). Violations of the Independence Axiom in Common Ratio Problems: An Experimental Test of Some Competing Hypotheses. Annals of Operations Research, Vol. 19, pp. 79-102.

Sornette, D. (2000). Stock market speculation: Spontaneous symmetry breaking of economic valuation. Physica A, Vol. 284, pp. 355-375. 
Stevens, J.P. (2002). Applied multivariate statistics for the social sciences (4th ed.). Hillsdale, NS: Erlbaum.

Sung, J., \& Hanna, S. D. (1996). Factors related to risk tolerance. Financial Counseling and Planning, Vol. 7, pp. 11-20.

Symmonds, M., Emmanuel, J.J., Drew, M.E., Batterham, R.L. \& Dolan, R.J. (2010). Metabolic State Alters Economic Decision Making under Risk in Humans. PLoS One, Vol. 5, No. 6, e11090

Tanaka, T., Camerer, C. \& Nguyen, Q. (2010). Risk and time preferences: linking experimental and household survey data from Vietnam. American Economic Review, Vol. 100, No. 1, pp. $557-571$.

Thomas, M., Sing, H., Belenky, G., Holcomb, H., Mayberg, H., Dannals, R., Wagner, H., Thorne, D., Popp, K., Rowland, L., Welsh, A., Balwinski, S. \& Redmond, D. (2000). Neural basis of alertness and cognitive performance impairments during sleepiness. I. Effects of $24 \mathrm{~h}$ of sleep deprivation on waking human regional brain activity. Journal of Sleep Research, Vol. 9, pp. 335-352.

Thurstone, L. (1927). Psychophysical Analysis. American Journal of Psychology, Vol. XXXVIII, pp. 368-389.

Thaler, R.H (1980). Towards a Positive Theory of Consumer Choice. Journal of Economic Behavior and Organization, Vol. 1, pp. 39-60.

Thaler, R.H. \& Johnson, E.J. (1990). Gambling with the house money and trying to break even: The effects of prior outcomes on risky choice. Management Science, Vol. 36, No. 6, pp. 643660.

Thayer, R.E. (1989). The biopsychology of mood and arousal. New York: Oxford University Press.

Torgerson, W. (1959). Theory and Methods of Scaling. Wiley. New York. 
Treffers, T., Koellinger, P.D. \& Picot, A. (2016). Do Affective States Influence Risk Preferences? Evidence from Incentive-Compatible Experiments. Schmalenbach Business Review, Vol. 17, pp. 309-335.

Tversky, A., \& Fox, C. R. (1995). Weighing risk and uncertainty. Psychological Review, Vol. 102, pp. 269-283.

Tversky A. \& Kahneman D. (1981). The Framing of Decisions and the Psychology of Choice. Science. New Series, Vol. 211, No. 4481, pp. 453-458.

Tversky, A. \& Kahneman, D. (1986). Rational Choice and the Framing of Decisions. The Journal of Business, Vol. 59, No. 4.

Tversky, A., \& Kahneman, D. (1992). Advances in prospect theory: cumulative representations of uncertainty. Journal of Risk \& Uncertainty, Vol. 5, 297-323.

Uhart, M., Oswald, L., McCaul, M.E., Chong, R. \& Wand, G.S. (2006). Hormonal responses to psychological stress and family history of alcoholism. Neuropsychopharmacology, Vol. 31, pp. 2255-2263.

Van de Venter, G., Michayluk, D. \& Davey, G. (2012). A longitudinal study of financial risk tolerance. Journal of Economic Psychology, Vol. 33, pp. 794-800.

Venkatraman, V., Chuah, L., Huettel, S.A. \& Chee, M.W.L. (2007). Sleep Deprivation Elevates Expectation of Gains and Attenuates Response to Losses Following Risky Decisions. SLEEP, Vol. 30, No. 5.

Van Der Zee, K., Thijs, M. \& Schaket, L. (2002). The Relationship of Emotional Intelligence with Academic Intelligence and the Big Five. European Journal of Personality, 16: 103-125.

von Neumann, J. \& Morgenstern, O. (1944). Theory of Games and Economic Behaviour. Princeton University Press.

Voors, M.J., Nillesen, E.E.M., Verwimp, P., Bulte, E.H., Lensink, R. \& Van Soest, D.P. (2012). Violent Conflict and Behavior: A Field Experiment in Burundi. The American Economic Review, Vol. 102, No. 2, pp. 941-964. 
Wakker, P. (2004a). Correcting Biases in Standard Gamble and Time Tradeoff Utilities. Medical Decision Making, SEP-OCT 2004.

Wakker, P. (2004b). Explaining the Characteristics of the Power (CRRA) Utility Family. Health Economics, Vol. 17, pp. 1329-1344.

Wakker, P. (2010). Prospect Theory, For Risk and Ambiguity. Cambridge University Press. pp.96-100.

Wakker, P. \& Deneffe, D. (1996). Eliciting von Neumann-Morgenstern utilities when probabilities are distorted or unknown. Management Science, 42 1131-1150.

Wang, H. \& Hanna, S. (1997). Does Risk Tolerance Decrease with Age? Financial Counseling and Planning, Vol. 8, No. 2, pp. 27-31.

Wegner, D.M. \& Wheatley, T. (1999). Apparent Mental Causation: Sources of the Experience of Will. American Psychologist, Vol. 54, No. 7, pp. 480-492.

Wu, G. \& Gonzalez, R. (1996). Curvature of the Probability Weighting Function. Management Science, Vol. 42, pp. 1676-1690.

Wu, G. \& Gonzalez, R. (1998). Common Consequence Conditions in Decision Making under Risk. Journal of Risk and Uncertainty, Vol. 16, pp. 115-139.

Wu, G., Zhang, J. \& Gonzalez, R. (2004). Decision under risk. In D. J. Koehler \& N. Harvey (Eds.), Blackwell handbook of judgment and decision making (pp. 399-423). Malden: Blackwell Publishing.

Xu, Y, Liu, Z., Zhao, J. \& Su, C. (2017). Weibo sentiments and stock return: A time-frequency view. PLoS ONE, Vol. 12, No. 7: e0180723. https://doi.org/10.1371/journal.pone.0180723

Yao, R., Hanna, S. D., \& Lindamood, S. (2004). Changes in financial risk tolerance, 1983-2001. Financial Services Review, Vol. 13, pp. 249-266.

Zhang, H., Zhou, R., Wang, J. \& Chen, X. (2016). An FMCDM approach to purchasing decision-making based on cloud model and prospect theory in e-commerce. International Journal of Computational Intelligence Systems, Vol. 9, No. 4, pp. 676-688. 